Homology, Homotopy and Applications, vol.1, No.1,1999, 1-78.

\title{
Groupoids and crossed objects in algebraic topology
}

\author{
Ronald Brown \\ School of Mathematics \\ University of Wales, Bangor \\ United Kingdom \\ r.brown@bangor.ac.uk \\ submitted by Hvedri Inassaridze \\ Received 19 October 1998, \\ in revised form, 22 December 1998
}

\begin{abstract}
This is an introductory survey of the passage from groups to groupoids and their higher dimensional versions, with most emphasis on calculations with crossed modules and the construction and use of homotopy double groupoids.
\end{abstract}

\section{Introduction}

This article is a slightly revised version of about nine hours of lectures given at the Summer School on the Foundations of Algebraic Topology, Grenoble, June 16 - July 4, 1997. The audience had been prepared in the previous week with basic lectures on algebraic topology. The intention of the lectures was to give a feel for what is going on, to encourage the listeners to read more widely in the area, and hopefully to suggest ways in which they could develop and apply some of these ideas in novel ways.

Published on 16 February 1999

1991 Mathematics Subject Classification: 55-02, 01A60, 55-03, 20L15, 18G55, 55Q05, 18D99

Key words and phrases: homotopy theory, groupoids, multiple groupoids, crossed modules, homotopy 2-types, crossed complexes, higher dimensional algebra.

(c) Ronald Brown 1999. Permission to copy for private use granted. 
The scope of this article is an introduction to the use of groupoids, higher dimensional groupoids, and related structures in homotopy theory. To this end it seems best to explain some motivation, proofs and applications reasonably fully and so I will concentrate on the use of crossed modules, double groupoids, and crossed complexes. Part of the aim is to show that these methods give not just theoretical understanding, but that they also lead to explicit computations of homotopical invariants which are unapproachable by other means. This is in some ways surprising, but in fact is a consequence of the attention paid to generalisations of the Van Kampen Theorem.

Since I have had to restrict the material, I could only hint at the work with Loday on crossed squares, tensor products, and the Van Kampen theorem for cubical diagrams of spaces given in $[44,45]$. Surveys of this material are given in $[21,20]$. Thus this article is still very much an introduction to the area.

\section{Contents}

1 Groupoids in homotopy theory 3

1.1 The fundamental groupoid . . . . . . . . . . . . . . . . . . . 4

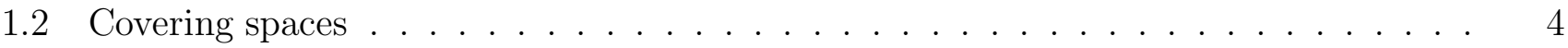

1.3 The Van Kampen Theorem . . . . . . . . . . . . . . . . . . . . . . . 5

1.4 Presentations of groupoids $\ldots \ldots \ldots \ldots \ldots \ldots$

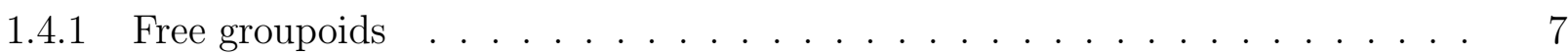

1.4 .2 Retractions ..................... 8

1.4.3 Normal subgroupoids and quotient groupoids . . . . . . . . . . . . . . 10

1.4.4 Presentations of groupoids . . . . . . . . . . . . . . . . . 10

1.5 Orbit spaces and orbit groupoids _ . . . . . . . . . . . . . . . . . 12

1.6 The category of groupoids . . . . . . . . . . . . . . . . . . . . . . . 12

1.7 The role of groupoids in algebraic topology and mathematics . . . . . . . . . . . . . 14

2 A search for higher order versions of the $\begin{array}{ll}\text { fundamental group in homotopy theory } & 16\end{array}$

3 Whitehead's work on crossed modules 18

3.1 The classifying space of a crossed module . . . . . . . . . . . . . . . . . . 20

4 The 2-dimensional Van Kampen Theorem 21 
5 Computation with crossed modules and second relative homotopy groups $\quad 23$

5.1 Induced crossed modules I: Algebra . . . . . . . . . . . . . . . . . . . . 23

5.2 Induced crossed modules II: Topological applications _ . . . . . . . . . . . . . . 28

5.3 Coproducts of crossed $P$-modules . . . . . . . . . . . . . . . . . . . . 33

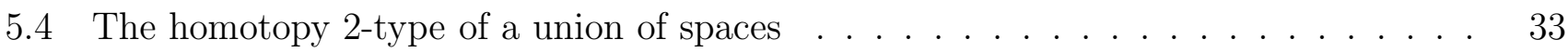

6 Homotopy double groupoids and the proof of the 2-dimensional Van Kampen Theorem $\quad 35$

6.1 Preliminaries on double groupoids . . . . . . . . . . . . . . . . . . . . 35

6.2 The homotopy double groupoid of a triple of spaces . . . . . . . . . . . . . . 38

6.3 General statement and proof of the 2-dimensional Van Kampen theorem . . . . . . 44

7 The category of crossed complexes $\quad 50$

7.1 The Generalised Van Kampen Theorem for Crossed Complexes . . . . . . . . . . . . 52

7.2 The nerve and classifying space of a crossed complex . . . . . . . . . . 56

7.3 Homotopies of morphisms of crossed complexes . . . . . . . . . . . . . . 60

7.4 Relation with chain complexes with operators . . . . . . . . . . . . . 62

7.5 Some computations of Postnikov invariants of crossed modules . . . . . . . . . . . . 64

$\begin{array}{lll}8 & \text { Further work } & 70\end{array}$ 


\section{Groupoids in homotopy theory}

The starting point of this study has been to consider the following extensions:

$$
\text { (groups) } \subset \text { (groupoids }) \subset \text { (multiple groupoids })
$$

The basic question is that of the significance of these extensions in the light of (i) the well recognised notion that groups form a central concept in mathematics, and (ii) the general importance of group theory for science.

A reason for regarding groups as significant is that they are considered to give the mathematics of reversible processes. This explains the connection with symmetry.

By a groupoid we mean simply a small category in which every morphism is an isomorphism. Thus a group may be considered as a groupoid with one object. The category of groupoids will now be written Gpd.

The extension from groups to groupoids starts in a formal sense with the desire to describe reversible processes which may traverse a number of states. So the group theory idea is say to consider a variety of journeys from say Bangor back to Bangor, whereas in groupoid theory one considers journeys between various cities in the UK, and observe that journeys can be continued or composed if and only if the starting point of one journey is the end point of the previous one. This naive viewpoint gives rise to the heretical suggestion that the natural concept is that of groupoid rather than that of group! In fact the groupoid idea is forced when one tries to structure a journey, i.e. to list the cities travelled.

It is interesting in this respect to note the view of Connes [58] that Heisenberg discovered quantum mechanics by considering the groupoid of quantum transitions rather than the group of symmetry.

The formal definition of groupoid can be considered a consequence of the legacy of Gauss, since it arose from Brandt's attempts to extend to quaternary forms Gauss' work on the composition of binary quadratic forms, which has a strong place in Disquitiones Arithmeticae. Bourbaki [12], p.153, cites this latter composition as an influential early example of a composition law which arose not from numbers, even taken in a broad sense, but from distant analogues ${ }^{1}$. Brandt found that each quaternary quadratic form of a given norm had a left unit and a right unit, and that two forms were composable if and only if the left unit of one was the right unit of the other. This led to his 1926 paper on groupoids. (A modern account of the work on composition of forms is given by Kneser et al. [93].) Groupoids were then used in the theory of orders of algebras. Curiously, groupoids did not form an example in Eilenberg and Mac Lane's basic 1945 paper on category theory.

\footnotetext{
${ }^{1}$ C'est vers cette même époque que, pour le premier fois en Algèbre, la notion de loi de composition s'étend, dans deux directions différents, à des élements qui ne présentent plus avec les $<<$ nombres $>>$ (au sens le plus large donné jusque-là à ce mot) que des analogies lointaines. La première de ces extensions est due à C.F.Gauss, à l'occasion de ses recherches arithmétiques sur les formes quadratiques...
} 


\subsection{The fundamental groupoid}

Groupoids appear in Reidemeister's 1932 book on topology [104], as the edge path groupoid, and for handling isomorphisms of a family of structures. The fundamental groupoid $\pi_{1}(X)$ of a space $X$ was well known by the 1950's. It consists of homotopy classes rel end points of paths in $X$, with the usual composition. Crowell and Fox remark in [60] that 'It is an interesting curiosity that the notion of groupoid gives a nice account of the idea of change of base point for the fundamental group.'

\subsection{Covering spaces}

Philip Higgins came to his view on the utility of groupoids from reading the account of [82] on covering spaces, and deciding this was mainly groupoid theory. He then proceeded to show the utility of using presentations of groupoids for applications to groups $[78,79,80]$. Basically, he showed that some of the topological versions of proofs of classical subgroup theorems (NielsenSchreier, Kurosch, Grusko, ... ) could be given an analogous algebraic proof, and generalised in the case of Grushko's theorem, using the notion of covering morphism of groupoids. This we now define.

First we define the star $\mathrm{St}_{H} x$ of a groupoid $H$ at an object $x$ of $H$ to be the set of all arrows of $H$ starting at $x$.

Definition 1.1 A morphism $p: H \rightarrow G$ of groupoids is a covering morphism if the induced maps of stars $\mathrm{St}_{H} x \rightarrow \mathrm{St}_{G} p x$ are bijective for all objects $x$ of $H$.

The category of covering morphisms of $G$, where the morphisms are the commutative triangles, is written $\operatorname{Gpd} \operatorname{Cov}(G)$.

Two other related categories are the category $\operatorname{Set}^{G}$ of functors $G \rightarrow$ Set, and the category $G$-Set of actions or operations of $G$ on sets. The objects of the latter category are triples $(A, w,$.$) where$ $A$ is a set, $w: A \rightarrow O b(G)$ is a function and . is a function which assigns to each $g: x \rightarrow y$ in $G$ and $a \in w^{-1} x$ and element $a . g \in w^{-1}(y)$ satisfying the usual action rules $a .1=a, a .(h g)=(a . g) . h$ whenever these are defined. The morphisms $f:(A, w,.) \rightarrow(B, v,$.$) of this category are functions$ $f: A \rightarrow B$ such that $v f=w, f(a . g)=(f a) . g$ whenever $a . g$ is defined.

The following is a basic result of groupoid theory:

Theorem 1.2 There are equivalences of categories

$$
\operatorname{GpdCov}(G) \simeq \operatorname{Set}^{G} \simeq G \text {-Set. }
$$

Let $\operatorname{Top} \operatorname{Cov}(X)$ be the category of covering maps of a space $X$. The main theorem of topological covering space theory is: 
Theorem 1.3 If the space $X$ admits a universal covering space then the fundamental groupoid functor

$$
\pi_{1}: \operatorname{Top} \operatorname{Cov}(X) \rightarrow \operatorname{Gpd} \operatorname{Cov}\left(\pi_{1}(X)\right)
$$

determines an equivalence of categories.

This gives a complete translation from topology to algebra. For proofs of these theorems, see for example [16].

When $G$ is a group, Theorem 1.2 can be given in a different form which Peter May has pointed out is useful in equivariant theory. We define the category $\operatorname{Or}(G)$ to have objects the sets $G / H$ of cosets for a subgroup $H$ of $G$ and to have morphisms the $G$-maps. Recall that a groupoid $G$ is connected if $G(x, y)$ is non empty for all objects $x, y \in G$.

Theorem 1.4 If $G$ is a group, then the category $\operatorname{Or}(G)$ is a skeleton of the full subcategory of $G$-Set consisting of transitive actions, and is also equivalent to the category of connected coverings of the group $G$.

Theorems 1.2, 1.3 also have versions for group objects in the respective categories. This is useful for discussing the existence of universal covering groups of non connected topological groups [47].

\subsection{The Van Kampen Theorem}

The start of my interest in groupoids came with the following theorem, due in this form to R.H. Crowell in 1959, but in fact for general open covers closed under finite intersection.

Theorem 1.5 (Van Kampen Theorem for the fundamental group) Let the space $X$ be the union of connected open sets $U, V$ with connected intersection $W$, and let $x \in W$. Then the diagram of group morphisms induced by inclusions

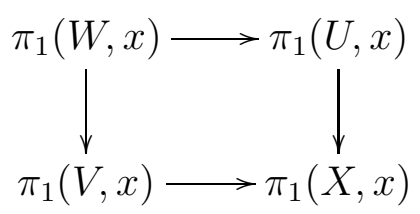

is a pushout of groups.

This is often written as an isomorphism of groups

$$
\pi_{1}(X, x) \cong \pi_{1}(U, x) *_{\pi_{1}(W, x)} \pi_{1}(V, x)
$$

It is in these forms that the theorem is used in [60], for example. 
The problem with this theorem is that it requires the connectivity condition and so does not yield the fundamental group of a circle, which in standard expositions is usually determined by the using the theory of covering spaces applied to the covering map $p: \mathrm{R} \rightarrow \mathrm{S}^{1}, \mathrm{t} \mapsto \exp (2 \pi \mathrm{it})$.

The main result of [15] was that a satisfactory result for non connected spaces could be obtained by replacing the single base point of the usual theory of the fundamental group by a set of base points chosen appropriately for the geometry. In particular, if the space $W$ above is not connected, we do not know in which component of $W$ to put a base point. We therefore avoid this choice by taking at least one 'base point' in each component of $W$. So we define for any set $A$ the fundamental groupoid $\pi_{1}(X, A)$ of $X$ on $A$ to be the full subgroupoid of $\pi_{1}(X)$ on the set $A \cap X$. That is, its object set is $A \cap X$ and the arrows are classes of paths in $X$ joining points of $A \cap X$, with the usual composition of arrows being the usual one.

This leads to the theorem:

Theorem 1.6 [15] Let the space $X$ be the union of open sets $U, V$ with intersection $W$, and let $A$ be a subset of $X$ meeting each path component of $U, V, W$. Then $A$ meets each path component of $X$ and the diagram of groupoid morphisms induced by inclusions

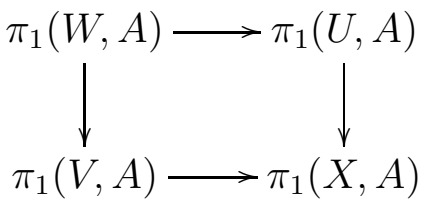

is a pushout of groupoids.

We do not give the proof here as a proof of a 2-dimensional version for general covers will be given later. An alternative proof is given in [33] as an application of cohomology with coefficients in a groupoid.

It is important to note that information on the way the components of $U, V, W$ intersect is naturally given in terms of graph theory. This nicely translates into algebraic information on groupoids. The representation of this information in terms of groups alone is much more awkward.

Grothendieck writes in 1985 [73]:

The idea of making systematic use of groupoids (notably fundamental groupoids of spaces, based on a given set of base points), however evident as it may look today, is to be seen as a significant conceptual advance, which has spread into the most manifold areas of mathematics.... In my own work in algebraic geometry, I have made extensive use of groupoids - the first one being the theory of the passage to quotient by a "preequivalence relation" (which may be viewed as being no more, no less than a groupoid in the category one is working in, the category of schemes say), which at once led me to the notion (nowadays quite popular) of the nerve of a category. The last time has been 
in my work on the Teichmüller tower, where working with a "Teichmüller groupoid" (rather than a "Teichmüller group") is a must, and part of the very crux of the matter $\ldots$

A survey of the use of groupoids up to 1986 is given in [19], and a more recent survey is [113].

\subsection{Presentations of groupoids}

In order to interpret the last theorem, one needs to be able to deal with presentations of groupoids. Here we can give only the indications of the theory.

The theory of groupoids may be thought of as an algebraic analogue of the theory of groups, but based on directed graphs rather than on sets. For some discussion of the philosophy of this, see [22].

\subsubsection{Free groupoids}

The term graph will always mean what is usually called a directed graph. A graph $X$ consists of two sets $\operatorname{Arr}(X), O b(X)$, of arrows and objects respectively of $X$, and two functions $s, t: \operatorname{Arr}(X) \rightarrow$ $O b(X)$, called the source and target maps. A morphism $f: X \rightarrow Y$ of graphs consists of two functions $\operatorname{Arr}(X) \rightarrow \operatorname{Arr}(Y), O b(X) \rightarrow O b(Y)$, which commute with the source and target maps. This defines the category Graph.

The underlying graph $U G$ of a groupoid $G$ has the same objects, arrows, source and target as $G$, but omits the composition and the function $O b(G) \rightarrow G$ giving the identities. This gives the forgetful functor $U:$ Gpd $\rightarrow$ Graph.

A basic construction in any algebraic theory is that of free objects. For groups, the free group functor $F:$ Set $\rightarrow$ Groups is left adjoint to the forgetful functor Groups $\rightarrow$ Set. In the case of groupoids, we may define the free groupoid functor to be the left adjoint $F:$ Graph $\rightarrow$ Gpd to the forgetful functor $U:$ Gpd $\rightarrow$ Graph. So if $X$ is a graph, then the free groupoid $F(X)$ on $X$ consists of a graph morphism $i: X \rightarrow U F(X)$ which is universal for morphisms from $X$ to the underlying graph of a groupoid.

The set of objects of $F(X)$ may be identified with $O b(X)$. There are several ways of explicitly constructing the set of arrows of $F(X)$. The usual way is as equivalence classes of composable words

$$
w=\left(x_{1}, \varepsilon_{1}\right) \ldots\left(x_{n}, \varepsilon_{n}\right), n \geqslant 0, x_{i} \in \operatorname{Arr}(X), \varepsilon= \pm
$$

together with empty words ()$_{a}, a \in O b(X)$, where the word $w$ is composable means that $t\left(x_{i}, \varepsilon_{i}\right)=$ $s\left(x_{i+1}, \varepsilon_{i+1}\right), i=1 \ldots n-1$, where

$$
s(x, \varepsilon)=\left\{\begin{array}{ll}
s x & \text { if } \varepsilon=+, \\
t x & \text { if } \varepsilon=-,
\end{array} \quad t(x, \varepsilon)= \begin{cases}t x & \text { if } \varepsilon=+, \\
s x & \text { if } \varepsilon=-.\end{cases}\right.
$$


The equivalence relation on words, and the composition, to obtain the free groupoid is defined in a manner analogous to the usual definition of free group, and the graph morphism $i: X \rightarrow F(X)$ sends an arrow $x$ to $[x]$, the equivalence class of the word $(x,+)$.

A groupoid $G$ is called connected if $G(a, b)$ is non empty for all $a, b \in O b(G)$. The maximal connected subgroupoids of $G$ are called the (connected) components of $G$.

If $a$ is an object of the groupoid $G$, then the set $G(a, a)$ inherits a group structure from the composition on $G$, and this is called the object group of $G$ at $a$ and is written also $G(a)$. The groupoid $G$ is called simply connected if all its object groups are trivial. If it is connected and simply connected, it is called 1-connected, or a tree groupoid.

A standard example of a tree groupoid is the indiscrete, or square, groupoid $I(S)$ on a set $S$. This has object set $S$ and arrow set $S \times S$, with $s, t: S \times S \rightarrow S$ being the first and second projections. The composition on $I(S)$ is given by

$$
(a, b)(b, c)=(a, c), a, b, c \in S .
$$

A graph $X$ is called connected if the free groupoid $F(X)$ on $X$ is connected, and is called a forest if every object group $F(X)(a)$ of $F(X), a \in O b(X)$, is trivial. A connected forest is called a tree. If $X$ is a tree, then $F(X)$ is a tree groupoid.

\subsubsection{Retractions}

Let $G$ be a connected groupoid. Let $a_{0}$ be an object of $G$. For each object $a$ of $G$ choose an arrow $\tau a: a_{0} \rightarrow a$, with $\tau a_{0}=1_{a_{0}}$. Then an isomorphism

$$
\phi: G \rightarrow G\left(a_{0}\right) \times I(O b(G))
$$

is given by $g \mapsto\left((\tau a) g(\tau b)^{-1},(a, b)\right), g \in G(a, b), a, b \in O b(G)$. The composition of $\phi$ with the projection yields a morphism $\rho: G \rightarrow G\left(a_{0}\right)$ which we call a deformation retraction, since it is the identity on $G\left(a_{0}\right)$ and is in fact homotopic to the identity morphism of $G$, though we do not elaborate on this fact here.

It is also standard $[16,8.1 .5]$ that a connected groupoid $G$ is isomorphic to the free product groupoid $G\left(a_{0}\right) * T$ where $a_{0} \in O b(G)$ and $T$ is any wide, tree subgroupoid of $G$. The importance of this is as follows.

Suppose that $X$ is a graph which generates the connected groupoid $G$. Then $X$ is connected. Choose a maximal tree $T$ in $X$. Then $T$ determines for each $a_{0}$ in $O b(G)$ a retraction $\rho_{T}: G \rightarrow G\left(a_{0}\right)$ and the isomorphisms

$$
G \cong G\left(a_{0}\right) * I(O b(G)) \cong G\left(a_{0}\right) * F(T)
$$

show that a morphism $G \rightarrow K$ from $G$ to a groupoid $K$ is completely determined by a morphism of groupoids $G\left(a_{0}\right) \rightarrow K$ and a graph morphism $T \rightarrow K$ which agree on the object $a_{0}$.

We shall use later the following proposition, which is a special case of [16, 6.7.3]: 
Proposition 1.7 Let $G, H$ be groupoids with the same set of objects, and let $\phi: G \rightarrow H$ be a morphism of groupoids which is the identity on objects. Suppose that $G$ is connected and $a_{0} \in O b(G)$. Choose a retraction $\rho: G \rightarrow G\left(a_{0}\right)$. Then there is a retraction $\sigma: H \rightarrow H\left(a_{0}\right)$ such that the following diagram, where $\phi^{\prime}$ is the restriction of $\phi$ :

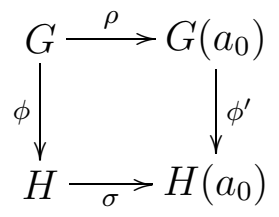

is commutative and is a pushout of groupoids.

This result can be combined with Theorem 1.6 to determine the fundamental group of the circle $S^{1}$.

Corollary 1.8 The fundamental group of the circle $S^{1}$ is a free group on one generator.

Proof We represent $S^{1}$ as the union of two semicircles $E_{+}^{1}, E_{-}^{1}$ with intersection $\{-1,1\}$. Then the fundamental groupoid $\pi_{1}\left(E_{+}^{1},\{-1,1\}\right)$ is easily seen to be isomorphic to the connected groupoid $\mathcal{I}$ with object set $\{-1,1\}$ and trivial object groups. In fact this groupoid is the free groupoid on one generator $\iota:-1 \rightarrow 1$. From previous results, we have a pushout diagram, in which $\{-1,1\}$ denotes the discrete groupoid on these objects,

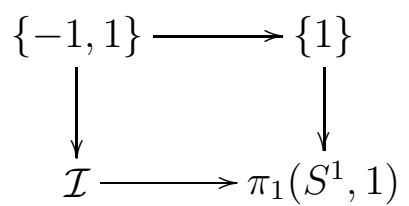

and the result follows by an easy universal argument.

Note that $S^{1}$ may be regarded as a pushout in the category of topological spaces

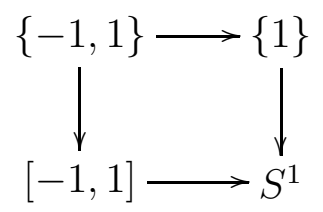

The correspondence between these last two diagrams was for me a major incentive to exploring the use of groupoids. Here we have a successful algebraic model of a space, but of a different type from that previously considered. An aspect of its success is that groupoids have structure in two dimensions, namely 0 and 1 , and this is useful for modelling the way spaces are built up using identifications in dimensions 0 and 1. 
Another advantage of this method is that it yields calculations for which the corresponding covering space theory is more awkward. One example is the fundamental group of the non Hausdorff manifold obtained by identifying all points of two copies of the unit interval except for the mid points. We find, as for $S^{1}$, that this space has fundamental group isomorphic to Z. Other examples are given in [16].

Another interesting aspect is that the groupoid $\mathcal{I}$ is finite, and it is easy to explore all its properties. By contrast, the integers form an infinite set, and discussion of its properties usually requires induction.

One problem posed by these results was to find analogous methods and results in higher dimensions.

\subsubsection{Normal subgroupoids and quotient groupoids}

Let $G$ be a groupoid. A subgroupoid $N$ of $G$ is called normal if $N$ is wide in $G$ (i.e. $O b(N)=O b(G)$ ) and for any objects $a, b$ of $G$ and $g$ in $G(b, a), g^{-1} N(b) g=N(a)$.

Let $\phi: G \rightarrow H$ be a morphism of groupoids. Then $\operatorname{Ker} \phi$ is the wide subgroupoid of $G$ whose elements are all $g$ in $G$ such that $\phi g$ is an identity of $H$ is a normal subgroupoid of $G$. If $O b(f)$ is injective then $\operatorname{Ker} \phi$ is totally disconnected, i.e. $(\operatorname{Ker} \phi)(a, b)=\emptyset$ if $a \neq b$.

A morphism $\phi: G \rightarrow H$ is said to annihilate a subgraph $X$ of $G$ if $\phi(X)$ is a discrete subgroupoid of $H$. Thus Ker $\phi$ is the largest subgroupoid of $G$ annihilated by $\phi$. The next proposition gives the existence of quotient groupoids.

Proposition 1.9 Let $N$ be a totally disconnected, normal subgroupoid of $G$. Then there is a groupoid $G / N$ and a morphism $p: G \rightarrow G / N$ such that $p$ annihilates $N$ and is universal for morphisms from $G$ which annihilate $N$.

Proof We define $O b(G / N)=O b(G)$. If $a, b \in O b(G)$ we define $(G / N)(a, b)$ to consist of all cosets $N(a) g, g \in G(a, b)$. The multiplication of $G$ is inherited by $G / N$, which becomes a groupoid.

The morphism $p: G \rightarrow G / N$ is the identity on objects, and on elements is defined by $g \mapsto$ $N(s g) g$. Clearly $p$ is a morphism and $\operatorname{Ker} p=N$.

The remainder of the proof is clear.

We call $G / N$ a quotient groupoid of $G$.

\subsubsection{Presentations of groupoids}

We now consider relations in a groupoid. Suppose given for each object $a$ of the groupoid $G$ a set $R(a)$ of elements of $G(a)$ - thus $R$ can be regarded as a wide, totally disconnected subgraph of $G$. The normal closure $N(R)$ of $R$ is the smallest wide normal subgroupoid of $G$ which contains $R$. 
This obviously exists since the intersection of any family of normal subgroupoids of $G$ is again a normal subgroupoid of $G$. Further, $N(R)$ is totally disconnected since the family of object groups of any normal subgroupoid $N$ of $G$ is again a normal subgroupoid of $G$.

Alternatively, $N=N(R)$ can be constructed explicitly. Let $a$ be an object of $G$. By a consequence of $R$ at $a$ is meant either the identity of $G$ at $a$, or any product

$$
\tau=g_{1}^{-1} r_{1}^{\varepsilon_{1}} g_{1} \ldots g_{n}^{-1} r_{n}^{\varepsilon_{n}} g_{n},
$$

in which $n \geqslant 1, g_{i} \in G\left(a_{i}, a\right)$ for some object $a_{i}$ of $G, \varepsilon_{i}= \pm 1$ and $r_{i}$ is an element of $R\left(a_{i}\right)$. Clearly, the set $N(a)$ of consequences of $R$ at $a$ is a subgroup of $G(a)$ and the family $N=(N(a): a \in O b(G)$ of these groups is a totally disconnected normal subgroupoid of $G$ containing $R$. Clearly $N=N(R)$.

The projection $p: G \rightarrow G / N(R)$ has the following universal property: if $f: G \rightarrow H$ is any morphism which annihilates $R$ then there is a unique morphism $f: G / N(R) \rightarrow H$ such that $f p=f$. We call $G / N(R)$ the groupoid $G$ with the relations $r=1, r \in R$.

In applications, we are often given $G, R$ as above and wish to describe the object groups of $G / N(R)$. These are determined by the following result.

Proposition 1.10 [16, 8.3.3] Let $G$ be connected, let $a_{0} \in O b(G)$ and let $\rho: G \rightarrow G\left(a_{0}\right)$ be a deformation retraction. Let $H=G / N(R)$. Then $H\left(a_{0}\right)$ is isomorphic to the group $G\left(a_{0}\right)$ with the relations

$$
\rho(r)=1, r \in R
$$

Proof The proof follows from Proposition 1.7, with $H=G / N$ and $\phi=p: G \rightarrow G / N$ the quotient morphism. Details are given in [16].

We can now indicate how to combine covering groupoids and presentations of groups to obtain results on groups.

Let $H$ be a subgroup of a group $G$. The $G$ acts on the set of cosets $G / H$ and so we obtain an associated covering morphism $p: K \rightarrow G$ with object set of $K$ equal to $G / H$ and object group $K(1)$, where 1 denotes the coset $H$, mapped by $p$ isomorphically to $H$. Suppose given a presentation $(X ; R)$ of the group $G$. Then we obtain a lifted presentation $(Y ; S)$ of the covering groupoid $K$, but $Y$ is here a connected directed graph. We can choose a retraction $\rho: K \rightarrow K(1)$ associated to this graph, for example by choosing a maximal tree in $Y$. This gives a presentation of $K(1)$, by the last proposition, and so a presentation of the subgroup $H$. This is the method used in [78, 79], and was in fact earlier used by Hasse in [75]. The details of the above argument require verifications on the lifting of presentations, and this we have to omit. It is worth pointing out that this methodology gives a stronger version of Grusko's theorem than had previously been known [79].

Again, Cayley graphs for presentations of groups are widely used, but it not so widely known that a convenient context for this is as the free generating graph $p^{-1}(X)$ of the free groupoid $K$ in the covering morphism $p: K \rightarrow F(X)$ determined by the kernel of $F(X) \rightarrow G$, together with 
the labelling given by the graph morphism $p^{-1}(X) \rightarrow X$. This description, with a corresponding 2-dimensional version, is exploited in [49].

Presentations of groupoids are used to obtain new results on cross ratio in projective geometry in $[56]$.

\subsection{Orbit spaces and orbit groupoids}

Another reason for choosing groupoids rather than groups concerns the equivariant theory, that is the case of actions of a group $\Gamma$ on a space $X$. If there is no fixed point for the action then information on the action cannot be reflected by any fundamental group $\pi_{1}(X, x)$. By contrast the group $\Gamma$ acts on the fundamental groupoid $\pi_{1}(X)$. The utility of this for calculations is shown below.

An action of the group $\Gamma$ on the space $X$ is called discontinuous if the stabiliser of each point of $X$ is finite, and each point $x$ in $X$ has a neighbourhood $V_{x}$ such that any element $\gamma$ of $\Gamma$ not in the stabiliser of $x$ satisfies $\left(\gamma \cdot V_{x}\right) \cap V_{x}=\emptyset$. An example of such an action is that of a finite group on a Hausdorff space.

If a group $\Gamma$ acts on a groupoid $G$, it is easy to define the notion of orbit groupoid as a $\Gamma$-morphism $p: G \rightarrow H$ of $\Gamma$-groupoids such that $\Gamma$ acts trivially on $H$ and $p$ is universal for this property: i.e. if $p^{\prime}: G \rightarrow H^{\prime}$ is any $\Gamma$-morphism of $\Gamma$-groupoids such that $\Gamma$ acts trivially on $H^{\prime}$, then there is a unique morphism $\phi: H \rightarrow H^{\prime}$ of groupoids such that $\phi p=p^{\prime}$.

The following theorem is due to R. Brown and P.J. Higgins and is proved in Chapter 9 of [16]. It gives a convenient formulation of work of A. Armstrong on the fundamental group of orbit spaces.

Theorem 1.11 If $X$ is a Hausdorff space on which the group $\Gamma$ acts discontinuously, and $X$ admits a universal covering space, then the induced morphism $p_{*}: \pi_{1} X \rightarrow \pi_{1}(X / \Gamma)$ makes $\pi_{1}(X / \Gamma)$ the orbit groupoid of $\pi_{1} X$ by the action of $\Gamma$.

This theorem gives a complete translation from topology to algebra, and it is then necessary to work out methods of calculating orbit groupoids. This has been done by Higgins and Taylor [81] and Taylor [111], whose work motivated the above theorem.

\subsection{The category of groupoids}

One way of evaluating the extension of categories

$$
\text { Groups } \subset \text { Gpd }
$$

from the viewpoint of the user is to consider any desirable properties which the larger category has which are not held by the smaller one. 
We have already seen that there is a notion of covering morphism of groupoids, which for the case of groups reduces simply to an isomorphism. There is also a notion of fibration of groupoids [17], which for the case of groups reduces to an epimorphism. The exact sequences arising from a fibration are useful even in group theory [76].

The categories Gpd and Groups are both complete and cocomplete, i.e. they admit limits and colimits. However a useful formal advantage of the bigger category is that for any groupoids $H, K$ there is also a groupoid $\operatorname{GPD}(H, K)$ whose objects are the morphisms $H \rightarrow K$ and whose arrows $f \rightarrow g$ are simply the natural equivalences. In the case $H, K$ are groups this means that an arrow $f \rightarrow g$ in $G P D(H, K)(f, g)$ is a pair $(f, k)$ such that $k \in K$ and for all $x \in H, g(x)=k^{-1} f(x) k$, that is, the arrows are conjugation by elements of $K$.

Theorem 1.12 For any groupoids $G, H, K$ there is a natural bijection

$$
\operatorname{Gpd}(G \times H, K) \cong \operatorname{Gpd}(G, G P D(H, K)) .
$$

Thus the category Gpd is cartesian closed. This result is a special case of the cartesian closedness of the category of small categories. A generalisation of this property to groupoids over a given groupoid has been exploited even to give information about groups [31].

This cartesian closed property is also useful in combination with the classifying space functor

$$
B: \text { Gpd } \rightarrow \text { Top. }
$$

To define this, recall that the nerve of a groupoid $G$ may be defined to be the simplicial set $N G$ such that $(N G)_{0}=O b(G)$ and whose $n$-simplices for $n \geqslant 1$ are the groupoid morphisms $\pi_{1}\left(\Delta^{n},\left(\Delta^{n}\right)_{0}\right) \rightarrow$ $G$, where $\left(\Delta^{n}\right)_{0}$ denotes the 0-skeleton of $\Delta^{n}$. Then $\pi_{n}(B G, x)=0$ for $n \geqslant 2, x \in O b(G)$, while $\pi_{1}(B G, O b(G)) \cong G$.

Let $X$ be a $C W$-complex, and let $G$ be a groupoid. It may be also proved that then there is a natural weak equivalence

$$
B\left(G P D\left(\pi_{1}(X), G\right)\right) \rightarrow(B G)^{X},
$$

where the latter space is the space of functions $X \rightarrow B G$ with the compact-open topology. This leads immediately on taking path components to a homotopy classification theorem for maps $X \rightarrow B G$, which may be written

$$
[X, B G] \cong\left[\pi_{1}(X), G\right]
$$

where the left hand side is the set of homotopy classes of maps, and the right hand side is the set of conjugacy classes of morphisms of groupoids. From this it may be shown that there is a map $X \rightarrow B \pi_{1}(X)$ inducing an isomorphism of fundamental groupoids. It is in this sense that groupoids model homotopy 1-types. These last two results are special cases of results on crossed complexes given in [42] (see section 10).

Another advantage of groupoids is that they have a satisfactory homotopy theory [91], since the groupoid $\mathcal{I}$ can be regarded as a model for groupoids of the unit interval. 


\subsection{The role of groupoids in algebraic topology and mathematics}

By this time it should be easy for you to be convinced that replacing the fundamental group by the fundamental groupoid leads to a more powerful and more elegant theory, and that groupoids give the natural and convenient exposition of the major facts of 1-dimensional homotopy theory. This was the viewpoint taken in my 1968 book [16].

This immediately raised questions about higher dimensions. If groupoids are useful in dimension 1 , how useful are they, or should they be, in higher dimensions?

There are two extreme possibilities.

1. Groupoids are useful in dimension 1 but are not more useful, or even useless, in higher dimensions.

2. Groupoids can become more useful with increasing dimension.

It was to attempt to decide on these possibilities that I began in the mid 1960s to investigate notions of higher dimensional groupoids. It turned out much more difficult to move from dimension 1 to dimension 2 than I expected and the first papers were not published till 1976 [50, 51].

It should be emphasised that for purely practical reasons the aim of this study could not be to solve other people's problems. The intention was to investigate this world, and to see what was there. It would clearly be a bonus if the theory solved old problems, and one could always hope, but the only way forward was to investigate the new territory. Fortunately, connections with classical concepts kept on appearing, and this was some comfort on a long road across a kind of desert before a lush countryside was reached. Also, at the beginning there were only a few clues as to the road to take. The only published information seemed to be the definition in Ehresmann's book published near that time [65] which gave a definition of double category and the example of the double category of commuting squares in a category.

There was also a gut feeling of the potential importance of such a study. The Principal Hypothesis was that the resulting theory would eventually come to bear to ordinary group theory a relation similar to that of many variable analysis to 1-variable analysis. This was the intention of this development of algebra to model geometry. The advantage of considering this hypothesis was that obstructions to it were also likely to be of interest, and that it suggested a range of areas for investigation.

Thus the study of multiple groupoids and their applications could be thought of as a higher dimensional, or many variable, theory, which would be expected to yield:

- a range of new algebraic structures, with new applications

- better understanding, from a higher dimensional viewpoint, of some phenomena in group theory 
- new algebraic understanding of the structure of certain geometric situations and hence new geometric theorems

- new computations with these objects, and hence also in the areas in which they apply.

It is thus reasonable to ask if these aims have already been achieved, and if not to ask how they might, if at all, be achieved.

The main applications of results derived from multiple groupoids have been in homotopy theory, as indicated here, differential topology (see for example [46, 113]), and combinatorial group theory (see for example [18, 83, 49]). A chief aim is to use these areas as test beds for the methods. For example, the fact that in homotopy theory one can achieve results not currently obtainable by other methods is an advertisement for these new local-to-global techniques, particularly in view of the difficulties in these areas. Indeed, a principal aim of this exposition is to give the background and intuition for some of these homotopical results. The overall aim of the investigation is is still to open up new modes of thought and questions.

The term 'higher dimensional group theory' [25] which was suggested in 1982 was also expected to be part of a wider theory of 'higher dimensional algebra', and this is now widely investigated (see for example $[4,3,2]$ ). This term also encompasses investigation of structures satisfying weaker axioms than the strict structures which are the main subject of these lectures. 


\section{A search for higher order versions of the fundamental group in homotopy theory}

One of the origins of the ideas for "higher dimensional group theory" is Poincaré's definition of the fundamental group, in his 1895 paper [100]. The motivation for the fundamental group in this paper seems to be from the notion of monodromy, that is the change in the value of a meromorphic function of many complex variables as it is analytically continued along a loop avoiding the singularities. This change in value depends only on the homotopy class of the loop. So Poincaré was led to the notion of the group $\pi_{1}(X, a)$ of homotopy classes of loops at $a$, where the group structure arises from composition of loops. He called this group the fundamental group, and the fundamental group $\pi_{1}(X, a)$, for a space $X$ and base point $a$, with its relation to covering spaces, surface theory, and the later combinatorial group theory, came to play an increasing rôle in the geometry and analysis of the next hundred years.

An early result was the relation with the first homology group, namely that for a connected simplicial complex $X$, the first homology group $H_{1}(X)$ is the fundamental group made abelian:

$$
H_{1}(X)=\pi_{1}(X, a)^{\mathrm{ab}} .
$$

It was thus clear that the non abelian fundamental group gave much more information than the first homology group. However, the homology groups were defined in all dimensions. So there was pressure to find a generalisation to all dimensions of the fundamental group.

According to [63], Dehn had some ideas on this in the 1920's, as would not be surprising. The first published attack on this question is the work of Cech. He submitted his paper on higher homotopy groups $\pi_{n}(X, a)$ to the International Congress of Mathematicians at Zurich in 1932. The story is that Alexandroff and Hopf quickly proved that these groups were commutative for $n \geqslant 2$, and then persuaded Cech to withdraw his paper, on the grounds that the higher homotopy groups clearly did not generalise the non abelian fundamental group. Presumably it was considered unreasonable that the structure proposed to model homotopy in dimensions larger than 1 should be simpler than that for 1 itself. All that appeared in the Proceedings of the Congress was a brief paragraph [57].

It is helpful to give the reason why the higher homotopy groups are Abelian. It is a corollary of the following result.

Theorem 2.1 Let $X$ be a set with two monoid structures $\circ_{1}, \circ_{2}$ each of which is a morphism for the other. Then the two structures coincide and are commutative.

Proof The condition that the structure $\circ_{1}$ is a morphism for $\circ_{2}$ is that the function

$$
\circ_{1}:\left(X, \circ_{2}\right) \times\left(X, \circ_{2}\right) \rightarrow\left(X, \circ_{2}\right)
$$

is a morphism of monoids, where $\left(X, \circ_{2}\right)$ denotes $X$ with the monoid structure $\circ_{2}$. This condition is equivalent to the statement that for all $x, y, z, w \in X$

$$
\left(x \circ_{2} y\right) \circ_{1}\left(z \circ_{2} w\right)=\left(x \circ_{1} z\right) \circ_{2}\left(y \circ_{1} w\right)
$$


which can be interpreted as saying that the diagram

$$
\left[\begin{array}{cc}
x & y \\
z & w
\end{array}\right] \downarrow_{1} 2
$$

has only one composition. This law is the well known interchange law.

We now use some special case of the interchange law. Let $e_{1}, e_{2}$ denote the identities for the structures $\circ_{1}, \circ_{2}$. We now consider the matrix

$$
\left[\begin{array}{ll}
e_{1} & e_{2} \\
e_{2} & e_{1}
\end{array}\right]
$$

This yields easily that $e_{1}=e_{2}$. We write then $e$ for $e_{1}$.

Now we consider the matrix composition

$$
\left[\begin{array}{ll}
x & e \\
e & w
\end{array}\right]
$$

Interpreting this in two ways yields

$$
x \circ_{1} w=x \circ_{2} w .
$$

So we write $\circ$ for $\circ_{1}$.

Finally we consider the matrix composition

$$
\left[\begin{array}{ll}
e & y \\
z & e
\end{array}\right]
$$

and find easily that $y \circ z=z \circ y$. This completes the proof.

Incidentally, it will also be found that associativity comes for free. We leave this to the reader.

This result seemed to kill any possibility of "higher dimensional group theory", or of any generalisations to higher dimensions of the fundamental group. In 1935, Hurewicz published the first of his celebrated notes on higher homotopy groups, and the latter are often referred to as the Hurewicz homotopy groups. The abelian higher homotopy groups came to be accepted, a considerable amount of work in homotopy theory has moved as far as possible from group theory and the non abelian fundamental group, and the original concern about the commutative nature of the higher homotopy groups came to be seen as a quirk of history.

The writing of the first edition of the book [16] made it natural to consider what happens to Theorem 2.1 if the word 'monoid' is replaced by 'category' or 'groupoid'. The proof then shows that a double category contains a family of abelian monoids. So double groupoids do not necessarily reduce to 'abelian' objects, and in fact it is now known that $n$-fold groupoids become increasingly 
complicated as $n$ increases. This suggested the interest in searching for higher dimensional versions of the fundamental groupoid, that is for higher homotopy groupoids.

Such an idea does not seem to have been put forward till its mention in the Introduction to [15]. The claim for a proof given there in 1966 of a higher dimensional Van Kampen Theorem might be considered premature, since there was no theorem formulated. In fact, the next eight or nine years of work were an idea of a proof in search of a theorem, and the move from dimension 1 to dimension 2 turned out to require a number of new ideas. It was however very satisfactory that the intuition for the proof found in 1966 was exactly embedded in the final theorem found with Philip Higgins in 1974, which was submitted in 1975 and published in 1978 [34].

\section{Whitehead's work on crossed modules}

Henry Whitehead was steeped in the combinatorial group theory of the 1930's, and much of his work can be seen as trying to extend the methods of group theory to higher dimensions, still keeping the interplay with geometry and topology. These attempts led to greatly significant work such as the theory of simple homotopy types. His ideas on crossed modules have taken longer to come into wide use but they can be regarded as equally significant.

One of his starting points was the Van Kampen Theorem for the fundamental group. This tells us how the fundamental group is affected by the attaching of a cell, or of a family of 2-cells, to a space. Namely, if $X=A \cup\left\{e_{i}^{2}\right\}_{i \in I}$, where the 2-cell $e_{i}^{2}$ is attached by a map which for convenience we suppose is $f_{i}:\left(S^{1}, 1\right) \rightarrow(A, x)$, then each $f_{i}$ determines an element $\phi_{i}$ in $\pi_{1}(A, x)$, and a consequence of the Van Kampen Theorem for the fundamental group is that the group $\pi_{1}(X, x)$ is obtained from the group $\pi_{1}(A, x)$ by adding the relations $\phi_{i}, i \in I$. This can be put in a more general format and easily proved using the results on presentations of groupoids given earlier.

The next problem is to determine the effect on the higher homotopy groups of adding cells to a space. If we could solve this in general, then we would in particular be able to calculate all homotopy groups of spheres. Work over the last 60 years has shown the enormous difficulty of this task.

One of the results Whitehead was able to obtain in 1941 [116] gave information on second homotopy groups of $X$ although it is only recently that it has been possible to develop this to a kind of algorithm [49]. His results were clarified and reformulated by him in two subsequent papers $[117,118]$ using the notion first of crossed module and then of free crossed module. This formulation became the key to higher order Van Kampen Theorems, as we shall see later.

We start with the basic definition of crossed module.

A crossed module is a morphism of groups $\mu: M \rightarrow P$ together with an action $(m, p) \mapsto m^{p}$ of $P$ on $M$ satisfying the two axioms

CM1) $\mu\left(m^{p}\right)=p^{-1} \mu(m) p$ 
CM2) $n^{-1} m n=m^{\mu n}$

for all $m, n \in M, p \in P$.

Standard algebraic examples of crossed modules are:

(i) an inclusion of a normal subgroup, with action given by conjugation;

(ii) the inner automorphism map $\chi: M \rightarrow$ Aut $M$, in which $\chi m$ is the automorphism $n \mapsto m^{-1} n m$;

(iii) the zero map $M \rightarrow P$ where $M$ is a $P$-module;

(iv) an epimorphism $M \rightarrow P$ with kernel contained in the centre of $M$;

(v) the free crossed P-module

$$
\partial: C(w) \rightarrow P
$$

determined by a function $w: R \rightarrow P$, where $R$ is a set. The group $C(w)$ is generated by $R \times P$ with the relations

$$
(r, p)^{-1}(s, q)^{-1}(r, p)\left(s, q p^{-1}(w r) p\right)
$$

the action is given by $(r, p)^{q}=(r, p q)$ and the boundary morphism is given by $\partial(r, p)=p^{-1}(w r) p$, for all $(r, p),(s, q) \in R \times P$. This construction will be seen later as a special case of the crossed $P$-module induced from the identity crossed $F(R)$-module $F(R) \rightarrow F(R)$ of the free group on $R$ by the morphism $w^{\prime}: F(R) \rightarrow P$ determined by $w$.

Simple consequences of the axioms for a crossed module $\mu: M \rightarrow P$ are:

\subsection{Im $\mu$ is normal in $P$.}

$3.2 \mathrm{Ker} \mu$ is central in $M$ and is acted on trivially by $\operatorname{Im} \mu$, so that Ker $\mu$ inherits an action of $M / \operatorname{Im} \mu$.

The major geometric example of crossed module can be expressed in two ways.

Let $(X, A, x)$ be a based pair of spaces. Whitehead showed that the boundary map

$$
\partial: \pi_{2}(X, A, x) \rightarrow \pi_{1}(A, x),
$$

together with the standard action of $\pi_{1}(A, x)$ on $\pi_{2}(X, A, x)$, has the structure of crossed module. Later Quillen put this in the form that if $F \rightarrow E \rightarrow B$ is a based fibration, then the induced morphism of fundamental groups $\pi_{1} F \rightarrow \pi_{1} E$ may be given the structure of crossed module.

Because of the first of these examples, it is convenient and sensible to regard crossed modules $\mu: M \rightarrow P$ as 2-dimensional versions of groups, with $P, M$ being respectively the 1- and 2dimensional parts. 
The category XMod of crossed modules has objects all crossed modules with morphisms the commutative diagrams

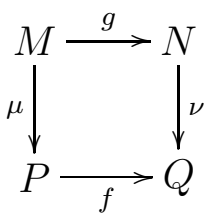

in which the vertical maps are crossed modules, and the pair $g, f$ preserve the action in the sense that for all $m \in M, p \in P$ we have $g\left(m^{p}\right)=(g m)^{f p}$.

Now we see that we have a functor

$$
\Pi_{2}: \text { (based pairs of spaces) } \rightarrow \text { XMod }
$$

which sends the based pair $(X, A, x)$ to the crossed module given in (10) above. (Later we shall formulate a groupoid version of this functor, but at the first shot we wish to keep things as simple as possible.)

\subsection{The classifying space of a crossed module}

In order to see the relevance of crossed modules to homotopy types we indicate a definition and state some properties of a classifying space functor $B[94,42]$ assigning to a crossed module $\mathcal{M}=$ $(\mu: M \rightarrow P)$ a connected, pointed $C W$-space $B \mathcal{M}$ with the following properties:

3.3 The homotopy groups of the classifying space of the crossed module $\mu: M \rightarrow P$ are given by

$$
\pi_{i}(B(M \rightarrow P)) \cong \begin{cases}\text { Coker } \mu & \text { for } i=1 \\ \text { Ker } \mu & \text { for } i=2 \\ 0 & \text { for } i>2\end{cases}
$$

3.4 The classifying space $B(1 \rightarrow P)$ is the usual classifying space $B P$ of the group $P$, and $B P$ is a subcomplex of $B(M \rightarrow P)$. Further, there is a natural isomorphism of crossed modules

$$
\Pi_{2}(B(M \rightarrow P), B P) \cong(M \rightarrow P) .
$$

3.5 (Loday [94],see also [42]) Let $X$ be a reduced $C W$-complex, let $Z$ be a connected space with $\pi_{2}(Z)=0$, and suppose given a map $f: Z \rightarrow X$ which is surjective on fundamental groups. Let $M \rightarrow P$ be the crossed module $\pi_{1}(F(f)) \rightarrow \pi_{1}(Z)$, where $F(f)$ is the homotopy fibre of $f$. Then there is a map

$$
X \rightarrow B(M \rightarrow P)
$$

inducing an isomorphism of $\pi_{1}$ and $\pi_{2}$. 
In this last result, it is easy to construct such a $Z$, for example it could be the 1-skeleton $X^{1}$ of $X$ so that $M \cong \pi_{2}\left(X, X^{1}\right)$. If $X=B(M \rightarrow P)$, for a crossed module $M \rightarrow P$, then we could take $Z=B P$.

It is in these senses that it is reasonable to say that crossed modules model all pointed homotopy 2-types. This result is due originally to Mac Lane and Whitehead [96] (they use the term 3-type for what is now called 2-type).

It is useful to indicate how $B(M \rightarrow P)$ is constructed as the geometric realisation of a simplicial set $K=\operatorname{Nerv}(M \rightarrow P)$. Later we shall give an elegant description of the simplices of this nerve, but here we shall give a sketch of their definition as in [42], and which in fact goes back to Blakers in [11].

First $K_{0}=0, K_{1}=P$. The 2-simplices of $K$ are quadruples $k=(m ; p, q, r)$ such that $m \in$ $M, p, q, r \in P$ and $\mu m=q p r^{-1}$ with $\partial_{0} k=p, \partial_{1} k=r, \partial_{2} k=q$. The 3-simplices of $K$ are quadruples $\left(k_{0}, k_{1}, k_{2}, k_{3}\right)$ of 2 -simplices such that if $k_{i}=\left(m_{i} ; p_{i}, q_{i}, r_{i}\right), i=0, \ldots, 3$, then

$$
m_{0}^{p_{3}} m_{2} m_{1}^{-1} m_{3}^{-1}=1
$$

and the edges of the 2 -simplices $k_{i}$ match up to form a 3 -simplex. For $n \geqslant 4$, an $n$-simplex of $K$ is an $(n+1)$-tuple of $(n-1)$-simplices of $K$, whose faces match up appropriately. In essence, the construction is defined by the so-called 'homotopy addition lemma'. We omit further details at this stage. (See section 7.2.) The construction in [94] is in terms of bisimplicial groups, but this is more difficult to use for homotopy classification results.

Clearly another problem is to give useful calculations of a crossed module representing the 2-type of a space. Our next result gives an essential ingredient for this.

\section{The 2-dimensional Van Kampen Theorem}

The 2-dimensional version of the Van Kampen Theorem is a theorem yielding pushouts of crossed modules or, more generally, colimits of crossed modules (see section 7.1 for the more general version).

First, we say the pair $(X, A)$ is connected if $A$ and $X$ are path connected and the induced map of fundamental groups $\pi_{1}(A, x) \rightarrow \pi_{1}(X, x)$ is surjective. This last condition is, with the connectivity of $A$, equivalent to saying that the homotopy fibre over $x$ of the inclusion $A \rightarrow X$ is path connected.

The following theorem will be proved in section 6.3 .

Theorem 4.1 (Brown and Higgins [34]) Suppose that the commutative diagram of based pairs of 
spaces

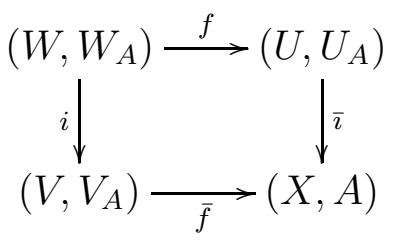

satisfies one of the two following hypotheses:

HYPOTHESIS A: the maps $i, f, \bar{\imath}, \bar{f}$ are inclusions of subspaces, $X$ is the union of the interiors of the sets $U$ and $V, W=U \cap V$, and $V_{A}=A \cap V, U_{A}=A \cap U, W_{A}=A \cap W$;

HYPOTHESIS B: the maps $i: W \rightarrow V, i_{A}: W_{A} \rightarrow V_{A}$ are closed cofibrations, $W_{A}=W \cap V_{A}$, and $X, A$ are the adjunction spaces $U \cup_{f} V, U_{A} \cup_{f_{A}} V_{A}$.

Suppose also that all the pairs $\left(U, U_{A}\right),\left(V, V_{A}\right),\left(W, W_{A}\right)$ are connected. Then:

(C) The pair $(X, A)$ is connected.

(I) The following diagram induced by inclusions

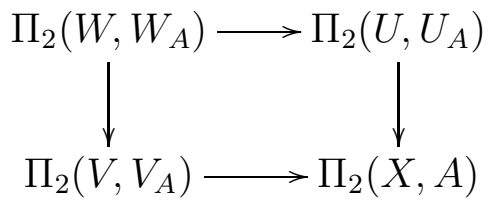

is a pushout of crossed modules.

The interest in this theorem is at least six fold.

- The theorem is a very useful computational tool and gives information unobtainable so far by other sources.

- The theorem is an example of a local-to-global theorem. Such theorems play an important rôle in mathematics and its applications.

- The theorem deals with non abelian objects, and so cannot be proved by traditional means of algebraic topology.

- The two available proofs use groupoid notions in an essential way.

- The existence of the theorem confirms the value of the crossed module concept, and of the methods used in its proof. We should be interested in algebraic structures for which this kind of result is true.

- It shows the difficulty of homotopy theory since one has, it appears, to go through all this just to determine the second homotopy groups of certain mapping cones.

A further point is that the proof we shall give later (section 6.3) does not assume the existence of pushouts in the category XMod. Instead the proof directly verifies the required universal property, and in fact the theorem deals with covers with any number of elements. 


\section{Computation with crossed modules and second relative homotopy groups}

The 2-dimensional van Kampen Theorem (Theorem 4.1) shows the interest in computing pushouts of crossed modules. A general treatment of this is in [34] but for explicit calculations there have so far been two main streams of results, namely induced crossed modules, and coproducts of crossed $P$-modules for a fixed $P$. We will be concerned here mainly with the former, partly because the results are newer and also because there is information and more references on the latter case in [83].

\subsection{Induced crossed modules I: Algebra}

Consider the situation of theorem 4.1, so that $X=U \cup V$ but in which we set $A=U$. Then we have a pushout of pairs in which $W=V \cap A$ :

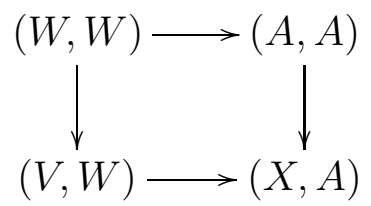

The inclusion of pairs $\varepsilon:(V, V \cap A) \rightarrow(V \cup A, A)$ is known as the excision map, since the smaller pair is obtained from the bigger one by 'excising' $A \backslash V$. A chief reason why homology is computable is that this map induces an isomorphism in relative homology in all dimensions if, for example, $U, V$ are open in $X$. By contrast, the excision map in relative homotopy is not an isomorphism even in dimension 2. In order to see what happens we interpret theorem 4.1 for this situation in the next section. This interpretation uses the notion of induced crossed module which was first described in [34], and which we now develop.

Definition 5.1 Let $\mathcal{M}=(\mu: M \rightarrow P)$ be a crossed module, which we abbreviate to $(M, P)$ when convenient, and let $\iota: P \rightarrow Q$ be a morphism of groups. The crossed module induced from $\mathcal{M}$ by $\iota$ is $\iota_{*} \mathcal{M}=\left(\partial: \iota_{*} M \rightarrow Q\right)$ defined by the pushout of crossed modules

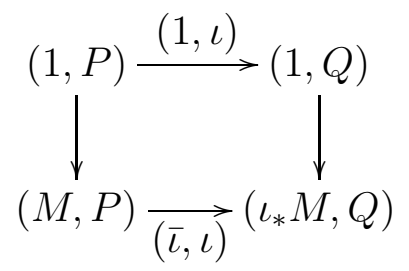

It can be proved from general considerations that the category XMod is complete and cocomplete, and in particular admits all pushouts. However, we will give below a presentation of the induced 
crossed module which can be used for specific calculations, and is also helpful for the calculation of colimits.

First we give another way of expressing the universal property of induced crossed modules using pullback crossed modules. Let $\iota: P \rightarrow Q$ be a morphism of groups. Let $\nu: N \rightarrow Q$ be a crossed $Q$-module. Let $\nu^{\prime}: \iota^{*} N \rightarrow P$ be the pullback of $N$ by $\iota$, so that $\iota^{*} N=\{(p, n) \in P \times N \mid \iota p=\nu n\}$, and $\nu^{\prime}:(p, n) \mapsto p$. Let $P$ act on $\iota^{*} N$ by $\left(p_{1}, n\right)^{p}=\left(p^{-1} p_{1} p, n^{\iota p}\right)$. The verification of the axiom CM1) is immediate, while CM2) is proved as follows:

Let $(p, n),\left(p_{1}, n_{1}\right) \in \iota^{*} N$. Then

$$
\begin{aligned}
(p, n)^{-1}\left(p_{1}, n_{1}\right)(p, n) & =\left(p^{-1} p_{1} p, n^{-1} n_{1} n\right) \\
& =\left(p^{-1} p_{1} p, n_{1}^{\nu n}\right) \\
& =\left(p^{-1} p_{1} p, n_{1}^{\iota p}\right) \\
& =\left(p_{1}, n_{1}\right)^{\nu^{\prime}(p, n)} .
\end{aligned}
$$

This can be expressed functorially. If $P$ is a group, then the category XMod/P of crossed $P$-modules is the subcategory of XMod whose objects are the crossed $P$-modules and in which a morphism $g: M \rightarrow N$ of crossed $P$-modules is a morphism of groups such that $\nu g=\mu$ and $g$ preserves the action in the sense that $g\left(m^{p}\right)=(g m)^{p}$, for all $m \in M, p \in P$.

Proposition 5.2 Pullback by $\iota$ defines a functor $\iota^{*}: \mathrm{XMod} / Q \rightarrow \mathrm{XMod} / P$ for which the induced module functor $\iota_{*}: \mathrm{XMod} / P \rightarrow \mathrm{XMod} / Q$ is a left adjoint.

Proof The proof is not hard, using the universal property of the pushout.

In terms of the last result, the universal property of induced crossed modules is the following. Let $\mu: M \rightarrow P, \gamma: C \rightarrow Q$ be crossed modules. In the diagram

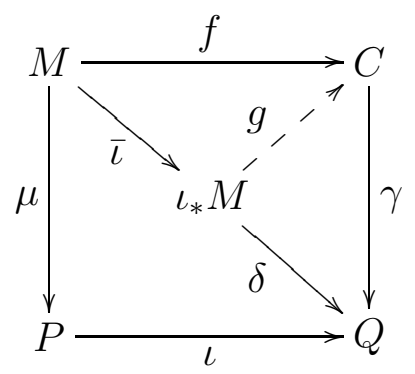

the pair $\bar{\iota}, \iota$ is a morphism of crossed modules such that for any crossed $Q$-module $\gamma: C \rightarrow Q$ and morphism of crossed modules $f, \iota$, there is a unique morphism $g: \iota_{*} M \rightarrow C$ of crossed $Q$-modules such that $g \bar{\iota}=f$.

It is a consequence of this universal property that if $\mu: F(R) \rightarrow F(R)$ is the identity crossed module for the free group $F(R)$ on a set $R$, and if $w: R \rightarrow Q$ is the restriction of $\iota$ to the set $R$, then $\iota_{*} F(R)$ is the free crossed module on $w$, in the sense of Whitehead [118] (see also [43, 83, 102]). Constructions of this free crossed module were given in these papers. 
The following presentation for crossed modules induced by a morphism $\iota$ is given in Proposition 8 of [34].

Proposition 5.3 Let $\mu: M \rightarrow P$ be a crossed P-module and let $\iota: P \rightarrow Q$ be a morphism of groups. Then the induced crossed $Q$-module $D=\iota_{*} M$ is generated, as a group, by the set $M \times Q$ with defining relations

(i) $\left(m_{1}, q\right)\left(m_{2}, q\right)=\left(m_{1} m_{2}, q\right)$,

(ii) $\left(m^{p}, q\right)=(m,(\iota p) q)$,

(iii) $\left(m_{1}, q_{1}\right)^{-1}\left(m_{2}, q_{2}\right)\left(m_{1}, q_{1}\right)=\left(m_{2}, q_{2} q^{-1}\left(\iota \mu m_{1}\right) q_{1}\right)$,

for $m_{1}, m_{2}, m \in M, q_{1}, q_{2}, q \in Q, p \in P$. The morphism $\partial: D \rightarrow Q$ is given by $\partial(m, q)=q^{-1}(\iota \partial m) q$, the action of $Q$ on $D$ by $\left(m, q_{1}\right)^{q}=\left(m, q_{1} q\right)$, and the canonical morphism $\bar{\iota}: M \rightarrow D$ by $\bar{\iota}(m)=$ $(m, 1)$.

Proof One verifies directly that this recipe defines a crossed $Q$-module and that $(\bar{\iota}, \iota):(M, P, \mu) \rightarrow$ $(D, Q, \partial)$ is a morphism of crossed modules with the required universal property.

The following is a consequence of this explicit presentation of the induced crossed module.

Corollary 5.4 If $\iota: P \rightarrow Q$ is the inclusion of the subgroup $P$ of $Q$, then the image of the boundary $\partial: \iota_{*} P \rightarrow Q$ of the crossed module induced from $1_{P}: P \rightarrow P$ is the normal closure of $P$ in $Q$.

Thus in the case of a subgroup $P$ of $Q$ the induced crossed module construction replaces the usual normal closure $N^{Q}(P)$ of $P$ in $Q$ by a group $\iota_{*} P$ which is in general larger than the normal closure $N^{Q}(P)$ and has additional structure satisfying a universal property. The kernel of the boundary map $\iota_{*} P \rightarrow Q$ has topological interest since it can be given as a second homotopy group (Corollary 5.18 ) but so far it has no independent algebraic construction.

We will need two more results from [34].

Proposition 5.5 [34, Proposition 9] If $\iota: P \rightarrow Q$ is a surjection, and $\mu: M \rightarrow P$ is a crossed $P$ module, then $\iota_{*} M \cong M /[M, K]$, where $K=\operatorname{Ker} \iota$, and $[M, K]$ denotes the subgroup of $M$ generated by all $m^{-1} m^{k}$ for all $m \in M, k \in K$.

The proof is a direct exercise in verifying that $\mu \iota$ determines a morphism $M /[M, K] \rightarrow Q$ which may be given the structure of crossed $Q$-module which satisfies the required universal property. We leave this for the reader.

The next results in this section come from [52]. We use the following term and notation. Let $P$ be a group and let $T$ be a set. We define the copower $P \vec{*} T$ to be the free product of groups $P_{t}, t \in T$, each with elements $(p, t), p \in P$, and isomorphic to $P$ under the map $(p, t) \mapsto p$. If $Q$ is a group, then $P \vec{*} Q$ will denote the copower of $P$ with the underlying set of the group $Q$. 
Proposition 5.6 If $\iota: P \rightarrow Q$ is an injection, and $\mu: M \rightarrow P$ is a crossed P-module, let $T$ be a right transversal of $\iota P$ in $Q$. Let $Q$ act on the copower $M \vec{*} T$ by the rule $(m, t)^{q}=\left(m^{p}, u\right)$, where $p \in P, u \in T$, and $t q=(\iota p) u$. Let $\delta: M \vec{*} T \rightarrow Q$ be defined by $(m, t) \mapsto t^{-1}(\iota \mu m) t$. Let $S$ be a set of generators of $M$ as a group, and let $S^{P}=\left\{x^{p}: x \in S, p \in P\right\}$. Then

$$
\iota_{*} M=(M \vec{*} T) / R
$$

where $R$ is the normal closure in $M \vec{*} T$ of the Peiffer commutators

$$
\langle(r, t),(s, u)\rangle=(r, t)^{-1}(s, u)^{-1}(r, t)(s, u)^{\delta(r, t)} \quad\left(r, s \in S^{P}, t, u \in T\right) .
$$

Proof Let $N=M \vec{*} T$. Proposition 10 of [34] yields that $\iota_{*} M$ is the quotient of $N$ by the subgroup $\langle N, N\rangle$ generated by $\left\langle n, n_{1}\right\rangle=n^{-1} n_{1}^{-1} n n_{1}^{\delta n}, n, n_{1} \in N$, and which is called in [43] the Peiffer subgroup of $N$. Now $N$ is generated by the set $\left(S^{P}, T\right)=\left\{\left(s^{p}, t\right): s \in S, p \in P, t \in T\right\}$, and this set is $Q$-invariant since $\left(s^{p}, t\right)^{q}=\left(s^{p p^{\prime}}, u\right)$ where $u \in T, p^{\prime} \in P$ satisfy $t q=\left(\iota p^{\prime}\right) u$. It follows from Proposition 3 of [43] that $\langle N, N\rangle$ is the normal closure of the set $\left\langle\left(S^{P}, T\right),\left(S^{P}, T\right)\right\rangle$ of basic Peiffer commutators.

Example 5.7 The dihedral crossed module We show how this works out in the following case, which exhibits a number of typical features. We let $Q$ be the dihedral group $D_{n}$ with presentation $\left\langle x, y: x^{n}=y^{2}=x y x y=1\right\rangle$, and let $M=P$ be the cyclic subgroup $C_{2}$ of order 2 generated by $y$. Let $C_{n}=\{0,1,2, \ldots, n-1\}$ be the cyclic group of order $n$. A right transversal $T$ of $C_{2}$ in $D_{n}$ is given by the elements $x^{i}, i \in C_{n}$. Hence $\iota_{*} C_{2}$ has a presentation with generators $a_{i}=\left(y, x^{i}\right), i \in C_{n}$, and relations given by $a_{i}^{2}=1, i \in C_{n}$, together with the Peiffer relations. Now $\delta a_{i}=x^{-i} y x^{i}=y x^{2 i}$. Further the action is given by $\left(a_{i}\right)^{x}=a_{i+1},\left(a_{i}\right)^{y}=a_{n-i}$. Hence $\left(a_{i}\right)^{\delta a_{j}}=a_{2 j-i}$, so that the Peiffer relations are $a_{j} a_{i} a_{j}=a_{2 j-i}$. It is well known that we now have a presentation of the dihedral group $D_{n}$, in which we get the standard presentation $\left\langle u, v: u^{n}=v^{2}=u v u v=1\right\rangle$ by setting $u=a_{0} a_{1}, v=a_{0}$, so that $u^{i}=a_{0} a_{i}$. Then

$$
\delta u=x^{2}, \delta v=y,
$$

so that $y$ acts on $\iota_{*} C_{2}$ by conjugation by $v$. However $x$ acts by

$$
u^{x}=u, v^{x}=v u \text {. }
$$

Note that this is consistent with the crossed module axiom CM2) since

$$
v^{x^{2}}=(v u)^{x}=v u u=u^{-1} v u .
$$

We call this crossed module the dihedral crossed module. It follows from these formulae that $\delta$ in the induced crossed module $\delta: D_{n} \rightarrow D_{n}$ is an isomorphism if $n$ is odd, and has kernel and cokernel isomorphic to $C_{2}$ if $n$ is even. In particular, if $n$ is even, then by results of section 5.2, $\pi_{2}\left(B D_{n} \cup \Gamma B C_{2}\right)$ has one non-trivial element which can be regarded as being represented by $u^{n / 2}$. 
Corollary 5.8 Assume $\iota: P \rightarrow Q$ is injective. If $M$ has a presentation as a group with g generators and $r$ relations, the set of generators of $M$ is P-invariant, and $n=[Q: \iota \mu(M)]$, then $\iota_{*} M$ has a presentation with gn generators and $r n+g^{2} n(n-1)$ relations.

Another corollary determines induced crossed modules under some abelian conditions. This result has useful applications. If $M$ is an abelian group, or $P$-module, and $T$ is a set we define the copower of $M$ with $T$, written $M \vec{\oplus} T$, to be the sum of copies of $M$ one for each element of $T$.

Corollary 5.9 Let $\mu: M \rightarrow P$ be a crossed $P$-module and $\iota: P \rightarrow Q$ a monomorphism of groups such that $M$ is abelian and $\iota \mu(M)$ is normal in $Q$. Then $\iota_{*} M$ is abelian and as a $Q$-module is just the induced $Q$-module in the usual sense.

Proof We use the result and notation of Proposition 5.6. Note that if $u, t \in T$ and $r \in S$ then $u \delta(r, t)=u t^{-1}(\iota \mu r) t=(\iota \mu m) u t^{-1} t=(\iota \mu m) u$ for some $m \in M$, by the normality condition. The Peiffer commutators given in Proposition 5.6 can therefore be rewritten as

$$
(r, t)^{-1}(s, u)^{-1}(r, t)(s, u)^{\delta(r, t)}=\left(r^{-1}, t\right)(s, u)^{-1}(r, t)\left(s^{m}, u\right) .
$$

Since $M$ is abelian, $s^{m}=s$. Thus the basic Peiffer commutators reduce to ordinary commutators. Hence $\iota_{*} M$ is the copower $M \vec{\oplus} T$, and this, with the given action, is the usual presentation of the induced $Q$-module.

Example 5.10 Let $M=P=Q$ be the infinite cyclic group, which we write $\mathrm{Z}$, and let $\iota: P \rightarrow Q$ be multiplication by 2 . Then $\iota_{*} M \cong \mathrm{Z} \oplus \mathrm{Z}$, and the action of a generator of $Q$ on $\iota_{*} M$ is to switch the two copies of $Z$. This result could also be deduced from known results on free crossed modules. However, our results show that we get a similar conclusion simply by replacing each $\mathrm{Z}$ in the above by for example $C_{4}$.

We also note the following theorem from [52], whose proof is omitted.

Theorem 5.11 [52] Let $\mu: M \rightarrow P$ be a crossed module and let $\iota: P \rightarrow Q$ be a morphism of groups. Suppose that $M$ and the index of $\iota(P)$ in $Q$ are finite. Then the induced crossed module $\iota_{*} M$ is finite.

The main difficulty in the proof is in the case when $\iota: P \rightarrow Q$ is injective.

Theorem 5.11 suggests that the computation of induced crossed modules should be accessible to symbolic computation. This has been realised as one of the functions in the GAP package XMOD [114] and in this way a number of calculations have been made [54].

The following theorem gives another useful instance of the determination of induced crossed modules. For any group $G$, let $I(G)$ denote its augmentation ideal, i.e. the kernel of the augmentation map ZG $\rightarrow$ Z. 
Theorem 5.12 [53] Let $M \subseteq P$ be normal subgroups of $Q$, so that $Q$ acts on $P$ and $M$ by conjugation. Let $\mu: M \rightarrow P, \iota: P \rightarrow Q$ be the inclusions and let $\mathcal{M}$ denote the crossed module $(\mu: M \rightarrow P)$ with the conjugation action. Then the induced crossed $Q$-module $\iota_{*} \mathcal{M}$ is isomorphic as a crossed $Q$-module to

$$
\left(\zeta: M \times\left(M^{\mathrm{ab}} \otimes I(Q / P)\right) \rightarrow Q\right)
$$

where for $m, n \in M, x \in I(Q / P)$ :

(i) $\zeta(m,[n] \otimes x)=m \in Q$;

(ii) the action of $Q$ is given by

$$
(m,[n] \otimes x)^{q}=\left(m^{q},\left[m^{q}\right] \otimes(\bar{q}-1)+\left[n^{q}\right] \otimes x \bar{q}\right) .
$$

The universal map $i: M \rightarrow M \times\left(M^{\mathrm{ab}} \otimes I(Q / P)\right)$ is given by $m \mapsto(m, 0)$, and if $(\beta, \iota)$ is a morphism from $\mathcal{M}$ to the crossed module $\mathcal{C}=(\chi: C \rightarrow Q)$, then the morphism

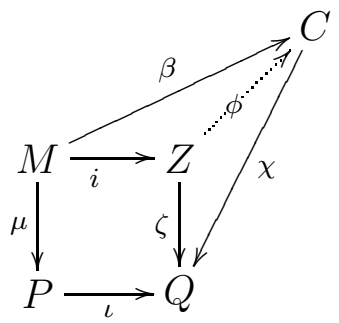

$\phi: M \times\left(M^{\mathrm{ab}} \otimes I(Q / P)\right) \rightarrow C$ induced by $\beta$ is, for $m, n \in M, q \in Q$, given by

$$
\phi(m,[n] \otimes(\bar{q}-1))=(\beta m)(\beta n)^{-1}\left(\beta\left(n^{q^{-1}}\right)\right)^{q} .
$$

The proof given in [53] is a direct verification of the universal property. The description of the action in (ii) of the last theorem is important and influences strongly the homotopy type of the classifying space $X$ of the induced crossed module. Correspondingly, you should avoid thinking that it is only the additive structure of the second homotopy group $\pi_{2}(X)$ which is of interest. I remember Whitehead saying that it was the action which especially fascinated the early workers in homotopy theory. Of course the deeper structures such as that given by the action, and by the crossed module itself, are of even more interest when there is some means of getting information on them in specific examples.

\subsection{Induced crossed modules II: Topological applications}

The following theorem is an easy consequence of Theorem 4.1. 
Theorem 5.13 Suppose that the commutative square

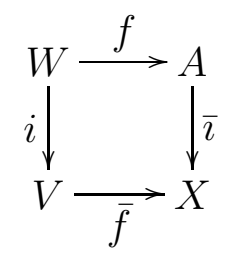

of based spaces satisfies one of the two following hypotheses:

HYPOTHESIS A: the maps $i, f, \bar{\imath}, \bar{f}$ are inclusions of subspaces, $W=V \cap A$, and $X$ is the union of the interiors of $V$ and $A$;

HYPOTHESIS B: the map $i$ is a closed cofibration and $X$ is the adjunction space $A \cup_{f} V$.

Suppose also that $(V, W)$ is 1-connected and $A$ is path connected. Then:

(C) $(X, A)$ is 1-connected;

(I) $\pi_{2}(X, A)$ is the crossed $\pi_{1}(A)$-module induced from $\pi_{2}(V, W)$ by the morphism $f_{*}: \pi_{1}(W) \rightarrow$ $\pi_{1}(A)$.

Proof Under these conditions we may take $A=U_{A}=U$ and $V_{A}=W_{A}=W$ in Theorem 4.1. Writing $P=\pi_{1}(W), Q=\pi_{1}(A), M=\pi_{2}(V, W)$, and $N=\pi_{2}(X, A)$ we find that

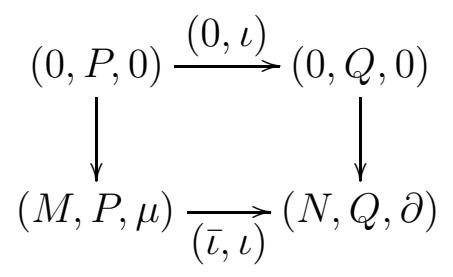

is a push-out of crossed modules, and this is equivalent to the assertion that $N$ is the induced module $\iota_{*} M$.

We shall discuss below the relation with what is usually called the 'homotopy excision theorem' (Example 5.19), but here move on to some other direct applications.

Corollary 5.14 (Relative Hurewicz Theorem in dimension 2) Suppose the pair $(V, W)$ is 1-connected. Then the space $V \cup C W$ is 1-connected and the group $\pi_{2}(V \cup C W)$ is isomorphic to the group $\pi_{2}(V, W)$ factored by the action of $\pi_{1}(W)$. 
Proof This follows from Theorem 5.13 with $f$ the inclusion $W \rightarrow C W$, Proposition 5.5, and the fact that $\pi_{2}(V \cup C W, C W) \cong \pi_{2}(V \cup C W)$.

This may seem odd since it is a relative Hurewicz Theorem without mention of homology. However, since $V \cup C W$ is 1-connected, the absolute Hurewicz Theorem implies $\pi_{2}(V \cup C W) \cong$ $H_{2}(V \cup C W)$ and this last group is isomorphic to $H_{2}(V, W)$ (by the excision theorem for homology).

It is difficult to envisage any proof of the following theorem except that given here.

Corollary 5.15 Let $X=A \cup_{f} C W$ be the mapping cone of a map $f: W \rightarrow A$, and suppose that $A, W$ are connected. Then $(X, A)$ is 1-connected and the crossed $\pi_{1}(A)$-module $\pi_{2}(X, A)$ is isomorphic to $\iota_{*} \pi_{1}(W)$, where $\iota: \pi_{1}(W) \rightarrow \pi_{1}(A)$ is induced by $f$. In particular, if $\pi_{2}(A)=0$, then $\pi_{2}(X)$ is isomorphic to the kernel of $\partial: \iota_{*} \pi_{1}(W) \rightarrow \pi_{1}(A)$.

Proof The statements about $\pi_{2}(X, A)$ follow from Theorem 5.13, and the final statement follows from the homotopy exact sequence of the pair $(X, A)$.

As pointed out earlier, in the case $P$ is a free group $F(R)$ on a set $R$, and $\mu$ is the identity, then the induced crossed module $\iota_{*} P$ is the free crossed $Q$-module on the function $\iota \mid R: R \rightarrow Q$. Thus the last corollary implies Whitehead's theorem:

Theorem 5.16 Let $X=A \cup\left\{e_{\lambda}^{2}\right\}_{\lambda \in \Lambda}$ be obtained from the connected space A by attaching 2-cells. Then the second relative homotopy group $\pi_{2}(X, A)$ may be described as the free crossed $\pi_{1}(A)$-module on the 2-cells.

Whitehead's original proof involved arguments of transversality and knot theory, and was developed over the papers $[117,116,118]$.

A considerable amount of work has been developed from this result, because of the connections with identities among relations, and methods such as transversality theory and "pictures" which developed from Whitehead's proof have proved successful $([43,102])$, particularly in the homotopy theory of 2-dimensional complexes [83]. However, the only route so far available to the wider geometric applications of induced crossed modules is Theorem 5.13.

We now give some other applications of Theorem 5.13.

Corollary 5.17 Let $\mu: M \rightarrow P$ be a crossed module, and let $\iota: P \rightarrow Q$ be a morphism of groups. Let $\beta: B P \rightarrow B(M \rightarrow P)$ be the inclusion. Consider the pushout

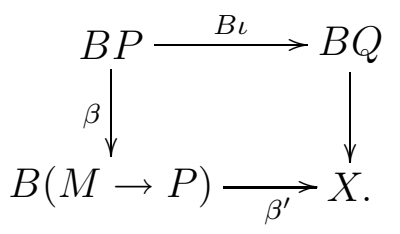


Then the fundamental crossed module of the pair $(X, B Q)$ is isomorphic to the induced crossed module $\iota_{*} M \rightarrow Q$, and there is a map

$$
X \rightarrow B\left(\iota_{*} M \rightarrow Q\right)
$$

inducing an isomorphism of $\pi_{1}, \pi_{2}$.

This shows that we have given a local-to-global computation of a homotopy 2-type. That this can be done at all is remarkable.

Further, for many specific finite examples the resulting induced crossed module can be computed completely.

Corollary 5.18 Let $\iota: P \rightarrow Q$ be a morphism of groups. Then the fundamental crossed module $\Pi_{2}\left(B Q \cup_{B \iota} C B P, B Q\right)$ is isomorphic to the induced crossed module $\iota_{*} P \rightarrow Q$, and hence the second homotopy group $\pi_{2}\left(B Q \cup_{B \iota} C(B P)\right)$ is isomorphic to $\operatorname{Ker}\left(\iota_{*} P \rightarrow Q\right)$. In particular, if $P$ is normal in $Q$, then this second homotopy group is isomorphic to $P^{\mathrm{ab}} \otimes I(Q / P)$.

Thus information about even such an apparently simple computation as a second absolute homotopy group of this mapping cone is tightly bound to information on crossed modules. There is at present no alternative description of this second homotopy group in algebraic terms. This highlights some basic difficulties of homotopy theory, and also suggests that homotopy theory is an essentially non abelian subject. The abelian homotopy groups, even as modules over the fundamental group, give only a pale shadow of the homotopical structures.

Part of the aim of finding models of these homotopical structures is the generic importance of the notion of homotopy, or deformation, as a means of classification. New methods developed here should prove their value in general areas of mathematics, in a manner similar to that of the general applications of homological algebra.

Example 5.19 Theorem 5.13 implies the homotopy excision theorem [89, p.211] in dimension 2. For suppose the based space $X$ is the union of subspaces $A, V$, with $A, V$ and $W=A \cap V$ all pathconnected. Assume either Hypothesis A or Hypothesis B' : $A$ and $V$ are closed and $i: W \rightarrow V$ is a cofibration. Let $\iota: \pi_{1}(W) \rightarrow \pi_{1}(A)$ be induced by inclusion. If $\pi_{1}(V, W)=0$, then $\pi_{1}(W) \rightarrow \pi_{1}(V)$ is surjective, and by Theorem 5.13, $\pi_{2}(X, A)=\iota_{*} \pi_{2}(V, W)$; this gives an algebraic description of the excision map $\epsilon: \pi_{2}(V, W) \rightarrow \pi_{2}(X, A)$. If also $\pi_{1}(A, W)=0$, then $\iota$ is surjective and we obtain from Proposition 5.5 the surjectivity of $\epsilon$ which is one part of the usual excision theorem; but we can also, by Theorem 5.13 and Proposition 5.5, state the further result that if $K=\operatorname{Ker} \iota$, then $K$ acts on $M=\pi_{2}(V, W)$, and $\operatorname{Ker} \epsilon=[M, K]$. Suppose further that

$$
\partial: \pi_{2}(A, W) \rightarrow \pi_{1}(W)
$$

is trivial (for example if $\pi_{2}(A, W)=0$ ); then $\iota: \pi_{1}(W) \rightarrow \pi_{1}(A)$ is an isomorphism and hence so also is $\epsilon$. This is the final part of homotopy excision under hypotheses slightly weaker than the usual ones. 
Example 5.20 Let $W, A, X$ be as in Corollary 5.15, and suppose that $f_{*}: \pi_{1}(W) \rightarrow \pi_{1}(A)$ is surjective with kernel $K$. An application of Proposition 5.5 to the conclusion of Theorem 5.13 gives $\pi_{2}(X, A)=\pi_{1}(W) /\left[\pi_{1}(W), K\right]$, and it follows from the homotopy exact sequence of the pair $(X, A)$ that there is an exact sequence

$$
\pi_{2}(A) \rightarrow \pi_{2}(X) \rightarrow K /\left[\pi_{1}(W), K\right] \rightarrow 0 .
$$

It follows from this exact sequence that if $W=K(P, 1)$ and $A=K(Q, 1)$, so that we have an exact sequence $1 \rightarrow K \rightarrow P \rightarrow Q \rightarrow 1$ of groups, then $\pi_{2}(X) \cong K /[P, K]$. Since $X$ is simply connected, we get the same result for $H_{2}(X)$. Now the homology exact sequence of the cofibre sequence $W \rightarrow A \rightarrow X$ gives an exact sequence

$$
H_{2}(P) \rightarrow H_{2}(Q) \rightarrow K /[P, K] \rightarrow H_{1}(P) \rightarrow H_{1}(Q) \rightarrow 0
$$

(originally due to Stallings). In particular if $P=F$, a free group, or one with $H_{2}(F)=0$, then we obtain an exact sequence

$$
0 \rightarrow H_{2}(Q) \rightarrow K /[F, K] \rightarrow F^{\mathrm{ab}} \rightarrow Q^{\mathrm{ab}} \rightarrow 0 .
$$

This gives the famous Hopf formula

$$
H_{2}(Q) \cong \frac{K \cap[F, F]}{[K, F]}
$$

which is one of the starting points of homological algebra.

As another application of (21) we note that if $\pi_{1}(A)=\pi_{2}(A)=0$, then $\pi_{2}(X)=\pi_{1}(W)^{a b}$. But $\pi_{2}(X) \cong H_{2}(X) \cong H_{1}(W)$, and so we obtain the absolute Hurewicz Theorem in dimension 1: if $W$ is connected, then $H_{1}(W) \cong \pi_{1}(W)^{a b}$.

The interest in this type of deduction of a well known and elementary result is as a model for other situations, where the notion of abelianisation is less transparent.

Example 5.21 Let $M$ be a normal subgroup of the group $P$ and let $\iota: P \rightarrow Q$ be a morphism of groups. Let $X$ be the homotopy pushout in the diagram

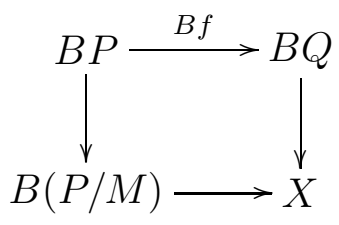

Then the homotopy 2-type of $X$ is described by the induced crossed module $\iota_{*} M \rightarrow Q$. This crossed module is determined completely in the case $\iota$ is an inclusion and $M, P$ are both normal in $Q$ in Theorem 5.12, from [53]. 
Remark 5.22 These results set the scene for later generalisations. In particular, the proof given here of the relative Hurewicz Theorem in dimension 2 led to its deduction in dimension $n$ from a Generalised Van Kampen Theorem for crossed complexes (see Corollary 7.9). This in turn suggested an $r$-adic Hurewicz Theorem as a deduction from an $r$-adic Van Kampen Theorem, via an $r$-cubical version of excision. This version of the Hurewicz Theorem $[45,20]$ has currently no other proof. Again, the above proof of the Hopf formula led to a higher dimensional version of the Hopf formula given by Brown and Ellis in [26], whose proof again uses the $r$-adic Hurewicz Theorem.

\subsection{Coproducts of crossed $P$-modules}

Here we give a brief review of another application of Theorem 4.1 to direct computation of homotopy groups.

Theorem 5.23 Let $M, N$ be normal subgroups of a group $P$, and let the space $X$ be given as the homotopy pushout in the following diagram, where BP denotes the classifying space of the discrete group $P$ :

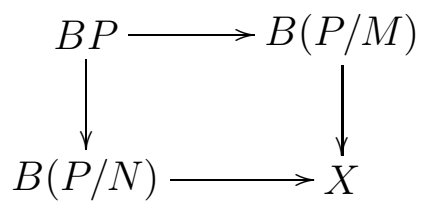

Then the first two homotopy groups of $X$ are given by

$$
\pi_{i}(X) \cong \begin{cases}P / M N & \text { if } i=1, \\ (M \cap N) /[M, N] & \text { if } i=2 .\end{cases}
$$

The proof uses an explicit description of the coproduct in the category XMod/P of crossed $P$-modules - for more details, see $[18,83]$. In fact the results in [18] are more complete since the crossed module representing the 2-type of the homotopy pushout $X$ in Theorem 5.23 is described completely as the coproduct of the two crossed $P$-modules $M \rightarrow P, N \rightarrow P$.

\subsection{The homotopy 2-type of a union of spaces}

Consider the situation of the 2-dimensional Van Kampen Theorem 6.3, and suppose that each of the spaces $U_{A}, V_{A}, W_{A}$ has trivial second homotopy group. Then the crossed modules $\Pi_{2}(U, U A)$, $\Pi_{2}\left(V, V_{A}\right), \Pi_{2}\left(W, W_{A}\right)$ determine the homotopy 2-types of the spaces $U, V, W$ respectively. Theorem 6.3 determines completely the crossed module $\pi_{2}(X, A)$ as the pushout of the other crossed modules. It is not true in general that $A$ then has trivial second homotopy group, as is clear from the previous sections. However if this does hold, then the homotopy 2-type of $X$ has been determined. This 
crossed module may in some cases be quite calculable - for example it might be finite. On the other hand, the second homotopy group, namely the kernel of the boundary map of $\Pi_{2}(X, A)$, may be difficult to calculate.

It is often held that the invariant of chief interest is the second homotopy group. This view may be because of the long familiarity of this invariant, and the previous difficulty of calculating an invariant of the homotopy 2-type. In any case, the virtue of algebraic models of homotopy types is that they fit naturally into algebraic constructions such as colimits and limits, and into more general theories such as homotopy coherence. 


\section{Homotopy double groupoids and the proof of the 2-dimensional Van Kampen Theorem}

\subsection{Preliminaries on double groupoids}

We now start on the definition and theory of the double groupoids used in the proof of the 2dimensional Van Kampen Theorem. Much of the following exposition is adapted from [34, 50].

By a double groupoid we shall always mean a 'special double groupoid with special connection' as defined in section 3 of [50]. We recall this definition, adopting a slightly different notation.

A double groupoid $G=\left(G_{2}, G_{1}, G_{0}\right)$ has, in the first place, the structure of a two-dimensional cubical complex. Thus there are face maps $\partial_{i}^{\alpha}: G_{n} \rightarrow G_{n-1}(\alpha=0,1, i=1,2, \ldots, n, n=1,2)$ and degeneracy maps $\epsilon_{i}: G_{n-1} \rightarrow G_{n}(i=1,2, \ldots, n, n=1,2)$ satisfying the usual cubical relations.

Next, for $n=1,2$, the pair $\left(G_{n}, G_{n-1}\right)$ has $n$ groupoid structures each with objects $G_{n-1}$ and arrows $G_{n}$. The groupoid 'in the ith direction' has initial and final maps $\partial_{i}^{0}, \partial_{i}^{1}: G_{n} \rightarrow G_{n-1}$, and its identity elements are the degenerate elements $\epsilon_{i} y$ for $y \in G_{n-1}$. The notation we use for these groupoid structures is as follows. Let $a, b \in G_{n}$ satisfy $\partial_{i}^{1} a=\partial_{i}^{0} b$. If $n=1$ (and therefore $i=1)$ the composite of the edges $a, b$ is written $a b$, and the identity edge $\epsilon_{1} y\left(y \in G_{0}\right)$ is written $e_{y}$, or $e$. If $n=2$ and $i=1$, the composite of the squares $a$ and $b$ is written $a \circ b$, with identity squares $1_{y}=\epsilon_{1} y\left(y \in G_{1}\right)$; we refer to this as 'vertical composition' of squares. If $n=2$ and $i=2$, the composite of $a$ and $b$ is written $a+b$, with identities $0_{y}=\epsilon_{2} y\left(y \in G_{1}\right)$; this is 'horizontal composition' of squares. If $a \in G_{1}$, the inverse of $a$ is written $a^{-1}$, while if $a \in G_{2}$, its inverses with respect to $\circ$ and + are written $a^{-1}$ and $-a$ respectively. We write $\odot_{y}$ for the doubly degenerate square $1_{e_{y}}=0_{e_{y}}\left(y \in G_{0}\right)$. We require also that the face maps $G_{2} \rightarrow G_{1}$ and the degeneracy maps $G_{1} \rightarrow G_{2}$ are morphisms of groupoids in the following sense:

(i) if $a+b$ is defined then $\partial_{1}^{\alpha}(a+b)=\left(\partial_{1}^{\alpha} a\right)\left(\partial_{1}^{\alpha} b\right)$;

(ii) if $a \circ b$ is defined then $\partial_{2}^{\alpha}(a \circ b)=\left(\partial_{2}^{\alpha} a\right)\left(\partial_{2}^{\alpha} b\right)$;

(iii) if $a b$ is defined then $0_{a b}=0_{a} \circ 0_{b}$ and $1_{a b}=1_{a}+1_{b}$.

The vertical and horizontal compositions of squares are related by the interchange law, namely, that if $a, b, c, d \in G_{2}$ then

$$
(a+b) \circ(c+d)=(a \circ c)+(b \circ d)
$$

whenever both sides are defined. It is convenient to use matrix notation for composition of squares. If $a \in G_{2}$, a subdivision of $a$ is defined to be a rectangular array $\left(a_{i j}\right)(1 \leqslant i \leqslant m, 1 \leqslant j \leqslant n)$ of elements of $G_{2}$ satisfying

$$
\begin{cases}\partial_{1}^{1} a_{i-1, j}=\partial_{1}^{0} a_{i, j} & (2 \leqslant i \leqslant m, 1 \leqslant j \leqslant n), \\ \partial_{2}^{1} a_{i, j-1}=\partial_{1}^{2} a_{i, j} & (1 \leqslant i \leqslant m, 2 \leqslant j \leqslant n),\end{cases}
$$


such that

$$
\left(a_{11}+a_{12}+\cdots+a_{1 n}\right) \circ\left(a_{21}+a_{22}+\cdots+a_{2 n}\right) \circ \cdots \circ\left(a_{m 1}+a_{m 2}+\cdots+a_{m n}\right)=a .
$$

We call $a$ the composite of the array $\left(a_{i j}\right)$ and write $a=\left[a_{i j}\right]$. The interchange law implies that if in the array $\left(a_{i j}\right)$ we partition the rows and columns into blocks $B_{k l}$ and compute the composite $b_{k l}$ of each block, then $a=\left[b_{k l}\right]$. We call the subdivision $\left(a_{i j}\right)$ a refinement of $\left(b_{k l}\right)$ in this case. Note that $a \circ b, a+c$ can also be written $\left[\begin{array}{l}a \\ b\end{array}\right],[a, c]$, and that the two sides of the interchange law can be written $\left[\begin{array}{ll}a & b \\ c & d\end{array}\right]$.

Before defining our further element of structure, we need one example of a double groupoid $[65,66]$. Let $H$ be a groupoid. The double groupoid $\square H$ of commuting squares in $H$ agrees with $H$ in dimension 1 and in dimension 2 consists of all quadruples $\alpha=\left(a_{a}^{b}{ }_{d}^{c}\right)$ of arrows of $H$ such that $a d=b c$, and where $\partial_{1}^{0} \alpha=b, \partial_{1}^{1} \alpha=d, \partial_{2}^{0} \alpha=a, \partial_{2}^{1} \alpha=c$. The two compositions of these commutative squares are the usual ones.

A thin structure on a double groupoid $G$ is a morphism $\Theta: \square G_{1} \rightarrow G$ of cubical sets such that $\Theta$ is the identity in dimensions 0 and 1 , and in dimension 2 preserves the two compositions (and hence preserves identities and inverses also). Thus the specification of a thin structure on a double groupoid $G$ has the effect of singling out a set of squares of $G$ with commuting boundary, which we shall call thin, with the two rules:

T1) any quadruple $(a, b, c, d)$ of elements of $G_{1}$ such that $a d=b c$ is the boundary of a unique thin square;

T2) any well defined composite of thin squares is thin.

This structure is crucial for our proof of the 2-dimensional Van Kampen Theorem, and for relating this proof to standard examples in homotopy theory.

There is clearly a category of double groupoids with thin structure, where the morphisms are morphisms of double groupoids preserving the thin structure. This category is written DbGpd. From now on the term 'double groupoid' will mean an object of this category.

We now give a complete algebraic source of such double groupoids. This requires generalising the notion of crossed module to the groupoid context.

Let $\Phi$ be a groupoid. A crossed $\Phi$-module consists of:

(i) a totally disconnected groupoid $M$ with the same object set as $\Phi$;

(ii) a morphism $\mu: M \rightarrow \Phi$ of groupoids which is the identity on objects; and 
(iii) an action of the groupoid $\Phi$ on the right of the groupoid $M$ via $\mu$.

This last condition means that if $x \in \Phi(a, b), m \in M(p)$, then $m^{x} \in M(b)$ and the usual laws of an action apply, namely $m^{1}=m,\left(m^{x}\right)^{y}=m^{x y},(m n)^{x}=m^{x} n^{x}$ whenever the terms are defined.

The axioms for a crossed module are:

CM1) $\mu\left(m^{x}\right)=x^{-1}(\mu m) x$,

CM2) $n^{-1} m n=m^{\mu n}$

for all $m, n \in M, x \in \Phi$ and whenever the terms are defined. Such a crossed $\Phi$-module is written $(M, \mu, \Phi)$ or $\mu: M \rightarrow \Phi$, or simply as $M$.

A morphism from a crossed module $\mu: M \rightarrow \Phi$ to a crossed module $\nu: N \rightarrow \Psi$ consists of a pair of morphisms of groupoids $f: \Phi \rightarrow \Psi, g: M \rightarrow N$ such that $\nu g=f \mu$ and $g\left(m^{x}\right)=(g m)^{f x}$ whenever $m^{x}$ is defined. This yields the category XModGpd of crossed modules and their morphisms.

Let $G$ be a double groupoid in the sense above. Then we obtain a crossed module $\gamma(G)=(\mu$ : $\left.M \rightarrow G_{1}\right)$ as follows. Its 1-dimensional part is just the groupoid $G_{1}$. Let $x \in G_{0}$. Then $M(x)$ consists of all squares $\alpha \in G_{2}$ such that $\partial_{1}^{0} \alpha=\partial_{2}^{0} \alpha=\partial_{2}^{1} \alpha=1_{x}$, and $\mu \alpha=\partial_{1}^{1} \alpha$. The multiplication in $M$ is given by + . The action of $G$ on $M$ is given by

$$
\alpha^{b}=-1_{b}+\alpha+1_{b} .
$$

The rule CM1) for a crossed module is clear, while CM2) can be proved by evaluating in two ways the composition

$$
\left[\begin{array}{ccc}
-1_{b} & \alpha & 1_{b} \\
-\beta & \odot & \beta
\end{array}\right]
$$

The functor $\lambda:$ XModGpd $\rightarrow$ DbGpd is defined as follows. Let $\mathcal{M}=(\mu: M \rightarrow \Phi)$ be a crossed module. Then $\lambda \mathcal{M}_{i}=\Phi_{i}$ for $i=0,1$. The squares of $\lambda \mathcal{M}$ consist of all quintuples $\left(m: a_{b}^{c} d\right)$ such that $m \in M, a, b, c, d \in \Phi$ and $\mu m=d^{-1} c^{-1} a b$. The boundary shell of such a quintuple is just the part excluding $m$ as should be expected, analogously to the case of commuting squares, and the two compositions are defined by:

$$
\begin{aligned}
& \left(m: a{ }_{b}^{c} d\right)+\left(n: d_{e}^{f} g\right)=\left(n m^{e}: a{ }_{b e}^{c f} g\right) \\
& \left(m: a{ }_{b}^{c} d\right) \circ\left(l: u_{v}^{b} w\right)=\left(m^{w} l: a u_{v}^{c} d w\right)
\end{aligned}
$$

The axioms for a double groupoid are trivial to verify except for the interchange law. This turns out to be equivalent to axiom CM2) for a crossed module. 
Finally the thin squares are those of the form

$$
\left(1: a \frac{c}{b} d\right) \text {. }
$$

Theorem 6.1 (Brown and Spencer [50], Brown and Higgins [35]) The functors $\gamma, \lambda$ defined above give an equivalence between the categories XMod and DbGpd.

An interesting aspect of the proof is that the hardest part is to construct the natural equivalence $\lambda \gamma \simeq 1$. Usually when one has an equivalence of categories, the result is clear once the functors are written down. That is not so in this case, and the problem gets even more difficult in higher dimensions. Essentially, the result says that if $G$ is a double groupoid (with thin structure) then $G$ can be reconstructed from the crossed module $\gamma G$ it contains.

\subsection{The homotopy double groupoid of a triple of spaces}

Throughout this section $X_{*}=\left(X, X_{1}, X_{0}\right)$ will be a triple of spaces, so that $X_{1}$ is a subspace of $X$ and $X_{0}$ is a subspace of $X_{1}$. We shall construct its 'homotopy double groupoid' $\rho\left(X_{*}\right)$.

First we construct $R=\left(R_{2}, R_{1}, R_{0}\right)$ where $R_{0}=X_{0}, R_{1}$ is the set of maps $(I, \dot{I}) \rightarrow\left(X_{1}, X_{0}\right)$, and $R_{2}$ is the set of maps $\left(I^{2}, \dot{I}^{2}, \ddot{I}^{2}\right) \rightarrow\left(X, X_{1}, X_{0}\right)$, where $\dot{I}^{2}$ is the set of edges and $\ddot{I}^{2}$ the set of vertices of the square $I^{2}$.

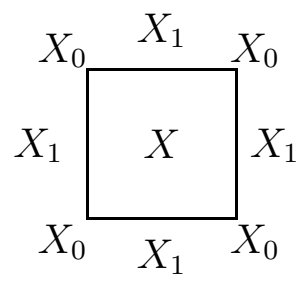

Then $R=R\left(X_{*}\right)$ has the structure of a two-dimensional cubical complex.

The set $R_{1}$ has its usual composition of paths in $X_{1}$ with end points in $X_{0}$. The set $R_{2}$ has two similar compositions. In more detail, for positive integers $m, n$ let $\varphi_{m, n}: I^{2} \rightarrow[0, m] \times[0, n]$ be the map $(x, y) \mapsto(m x, n y)$. An $m \times n$ subdivision of a square $\alpha: I^{2} \rightarrow X$ is a factorization $\alpha=\alpha^{\prime} \circ \varphi_{m, n}$; its parts are the squares $\alpha_{i j}: I^{2} \rightarrow X$ defined by

$$
\alpha_{i j}(x, y)=\alpha^{\prime}(x+i-1, y+j-1) .
$$

We then say that $\alpha$ is the composite of the squares $\alpha_{i j}$, and we write $\alpha=\left[\alpha_{i j}\right]$. Similar definitions apply to paths and cubes.

Such a subdivision determines a cell-structure on $I^{2}$ as follows. The intervals $[0, m],[0, n]$ have cell-structures with integral points as 0 -cells and the intervals $[i, i+1]$ as closed 1-cells. Then 
$[0, m] \times[0, n]$ has the product cell-structure which is transferred to $I^{2}$ by $\varphi_{m, n}^{-1}$. We call the 2-cell $\varphi_{m, n}^{-1}([i-1, i] \times[j-1])$ the domain of $\alpha_{i j}$.

We use the same notation for degenerate squares as in the previous section. If $\alpha \in R_{2}$, then $\alpha^{-1},-\alpha$ denote respectively the elements of $R_{2}$ defined by $(r, s) \mapsto \alpha(1-r, s), \quad(r, s) \mapsto \alpha(r, 1-s)$.

The double groupoid $\rho=\left(\rho_{2}, \rho_{1}, \rho_{0}\right)$ is in dimensions 0 and 1 just the fundamental groupoid $\pi_{1}\left(X_{1}, X_{0}\right)$, so that $\rho_{0}=X_{0}$, and $\rho_{1}$ consists of homotopy classes rel vertices of maps $(I, \dot{I}) \rightarrow$ $\left(X_{1}, X_{0}\right)$, with the usual composition. For dimension 2, the elements of $\rho_{2}$ are homotopy classes rel vertices of maps $\left(I^{2}, \dot{I}^{2}, \ddot{I}^{2}\right) \rightarrow\left(X, X_{1}, X_{0}\right)$.

We write $\equiv$ for the relation of homotopy on $R_{1}$ and $R_{2}$ and call it f-homotopy (or filter homotopy), to distinguish it from homotopy of maps $I \rightarrow X_{1}$ or $I^{2} \rightarrow X$ which we write $\simeq$. The class in $\rho_{i}$ of an element $\theta$ of $R_{i}$ is written $\bar{\theta}$.

An element $\bar{\theta}$ of $\rho_{2}$ is called thin if it has a representative $\theta$ such that $\theta\left(I^{2}\right)$ is contained in $X_{1}$.

Proposition 6.2 The compositions on $R\left(X_{*}\right)$ induce compositions on $\rho\left(X_{*}\right)$ which with the above thin elements make $\rho\left(X_{*}\right)$ a double groupoid.

Proof Since $\rho_{1}$ is just the fundamental groupoid, the major task is to prove that the compositions on $R_{2}$ are inherited by $\rho_{2}$.

Let $\bar{\alpha}, \bar{\beta} \in \rho_{2}$ satisfy $\partial_{2}^{1} \bar{\alpha}=\partial_{2}^{0} \bar{\beta}$. Then there is a square $h$ in $X_{1}$ with $\gamma=[\alpha h \beta]$ defined and with $\partial_{1}^{0} h, \partial_{1}^{1} h$ constant paths in $X_{0}$. We let $\bar{\alpha}+\bar{\beta}=\bar{\gamma}$ and prove this addition to be well defined.

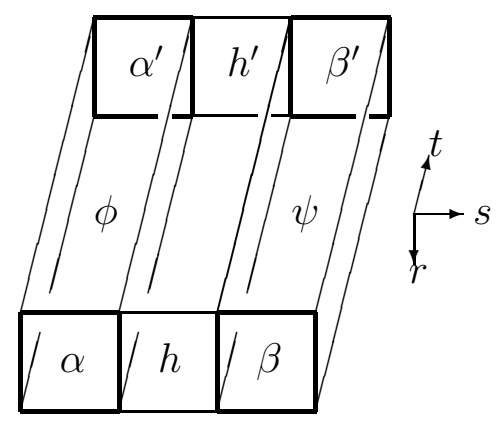

Figure 1

Let $\gamma^{\prime}=\left[\alpha^{\prime} h^{\prime} \beta^{\prime}\right]$ be alternative choices. Then there exist f-homotopies $\phi_{t}: \alpha \equiv \alpha^{\prime}, \psi_{t}: \beta \equiv \beta^{\prime}$. Let $k: I \times \dot{I}^{2} \rightarrow X_{1}$ be given by $(r, s, 0) \mapsto h(r, s),(r, s, 1) \mapsto h^{\prime}(r, s),(r, 0, t) \mapsto \phi_{t}(r, 1),(r, 1, t) \mapsto$ $\psi_{t}(r, 0)$. In terms of Figure 1, in which the thin lines denote edges on which the maps are constant, $k$ is the map defined on the four side faces of the central hole. But $k$ is constant on the edges of the bottom face, since all the homotopies are rel vertices. So $k$ extends over $\{1\} \times I^{2} \rightarrow X_{1}$ extending $k$ to five faces of $I^{3}$. By retracting $I^{3}$ onto these five faces we obtain a further extension $k: I^{3} \rightarrow X_{1}$. The composite cube $[\phi k \psi]$ is an f-homotopy $\gamma \equiv \gamma^{\prime}$ as required: the key point is that the extension maps the top face of the middle cube into $X_{1}$, since that is true for all the other faces. 
It is now easy to see that this addition makes $\left(\rho_{2}, \rho_{1}\right)$ a groupoid with initial and final maps $\partial_{2}^{0}, \partial_{2}^{1}$ and identity elements $0_{s}$, where $s \in \rho_{1}$. A similar procedure gives the other groupoid structure.

To verify the interchange law, suppose that $\bar{\alpha}, \bar{\beta}, \bar{\gamma}, \bar{\delta} \in \rho_{2}$ are such that either of the two composites

$$
\left[\begin{array}{ll}
\bar{\alpha} & \bar{\beta} \\
\bar{\gamma} & \bar{\delta}
\end{array}\right]
$$

are defined. Then there are maps $h, h^{\prime}, k, k^{\prime}: I^{2} \rightarrow X_{1}$ such that the following compositions are defined except for the middle element:

$$
\left[\begin{array}{lll}
\alpha & h & \beta \\
k & & k^{\prime} \\
\gamma & h^{\prime} & \delta
\end{array}\right]
$$

However because all homotopies are rel vertices, the middle square can be filled by a constant map to give a composable array. Evaluating this in two ways gives the interchange law.

Clearly a thin element of $\rho_{2}$ has commuting boundary, and any commuting boundary has a thin filler. We have to prove this filler is unique.

Let $f_{0}, f_{1}:\left(I^{2}, \ddot{I}^{2}\right) \rightarrow\left(X_{1}, X_{0}\right)$ be two maps which agree on $\dot{I}^{2}$. Define $F: I^{2} \times I \rightarrow X_{1}$ as follows. On $I^{2} \times \dot{I}, F$ is defined by $f_{0}, f_{1}$, while on three of the remaining faces of $I^{3}=I^{2} \times I, F$ is given by constant homotopies of the corresponding edges of $I^{2}$. Now use a retraction of $I^{3}$ onto these five faces to extend these maps to give an f-homotopy $F: f_{0} \equiv f_{1}$ as required.

Finally, it is clear from the homotopy extension property that any composite of thin elements is thin.

The next proposition is one of the keys to our work. It shows that double groupoids allow a convenient expression for the homotopy addition lemma in dimension 2 . To this end, we introduce the following convenient notation for certain thin elements:

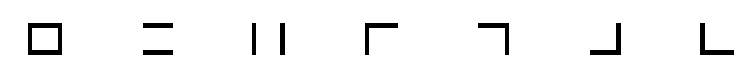

Here a thick line denotes an identity edge. Because a thin element is entirely determined by its boundary, which also must be commutative, it is easy to deduce relations between the other edges of these thin squares. Also, the two rules for thin elements immediately lead to equations such as:

$$
\begin{aligned}
& \left.\left[\begin{array}{ll}
\lrcorner & \mathrm{I} \\
\Xi & \rfloor
\end{array}\right]=\right\rfloor \\
& {\left[\begin{array}{ll}
\Gamma & \lrcorner
\end{array}\right]=\mathrm{I} I}
\end{aligned}
$$

The first of these is known as the transport law since it was borrowed from a related law for path connections in differential topology [50].

Part of the point of this is that 2-dimensional algebra is more complicated than 1-dimensional algebra, as one would expect. In particular, in dimension 1 the only 'thin' elements are identities, 
which correspond to constant paths. In dimension 2 we can not only stand still or turn around as in 1-dimensional algebra, we can also turn left or right. This opens up quite new algebraic possibilities.

The last four thin elements of (27) can in $\rho_{2}$ be defined by specific squares, of which the basic one is called a 'connection' $\Gamma: R_{1} \rightarrow R_{2}$. It is given by

$$
\Gamma(\sigma):(s, t) \mapsto\left\{\begin{array}{l}
\sigma(s) \text { if } 0 \leqslant t \leqslant s \leqslant 1, \\
\sigma(t) \text { if } 0 \leqslant s \leqslant t \leqslant 1 .
\end{array}\right.
$$

Clearly $\partial_{1}^{0} \Gamma(\sigma)=\partial_{2}^{0} \Gamma(\sigma)=\sigma$ and $\partial_{1}^{1} \Gamma(\sigma)=\partial_{2}^{1} \Gamma(\sigma)=\epsilon_{0} y$ where $y=\partial_{1}^{1} \sigma$. Also $\Gamma$ satisfies the transport law (28).

If $h: I^{3} \rightarrow X$ is a cube in $X$, then its faces are, as usual, given by $\partial_{i}^{\alpha} h=h \circ \eta_{i}^{\alpha}$, where $\eta_{i}^{\alpha}\left(x_{1}, x_{2}\right)=\left(y_{1}, y_{2}, y_{3}\right)$, the $y_{j}$ being defined by $y_{j}=x_{j}$ for $j<i, y_{i}=\alpha$, and $y_{j}=x_{j-1}$ for $j>i$. Also let $\tilde{\eta}_{1}^{\alpha}\left(x_{1}, x_{2}\right)=\left(\alpha, x_{2}, x_{1}\right)$.

Proposition 6.3 (the homotopy addition lemma). Let $X_{*}, \rho$ be as in Proposition 6.2. Let $h$ be a cube in $X$ with edges in $X_{1}$ and vertices in $X_{0}$, and let the elements $a_{\alpha}, b_{\alpha}, c_{\alpha}$ of $\rho_{2}$ represented by its faces be respectively the classes of $h \circ \tilde{\eta}_{1}^{\alpha}, h \circ \eta_{2}^{\alpha}, h \circ \eta_{3}^{\alpha}(\alpha=0,1)$. Then

$$
c_{1}=\left[\begin{array}{ccc}
\ulcorner & \alpha_{0}^{-1} & \neg \\
-b_{0} & c_{0} & b_{1} \\
\llcorner & a_{1} & \lrcorner
\end{array}\right]
$$

in $\rho_{2}$ where the corner elements are thin elements as above.

Proof Consider the maps $\varphi_{0}, \varphi_{1}: I^{2} \rightarrow I^{3}$ defined by

$$
\varphi_{0}=\left[\begin{array}{ccc}
-\Gamma^{-1} & \left(\tilde{\eta}_{1}^{0}\right)^{-1} & \Gamma^{-1} \\
-\eta_{2}^{0} & \eta_{3}^{0} & \eta_{2}^{1} \\
-\Gamma & \tilde{\eta}_{1}^{1} & \Gamma
\end{array}\right], \quad \varphi_{1}=\left[\begin{array}{ccc}
-\Gamma^{-1} & 1 & \Gamma^{-1} \\
0 & \eta_{3}^{1} & 0 \\
-\Gamma & 1 & \Gamma
\end{array}\right] .
$$

Then $\varphi_{0}, \varphi_{1}$ agree on $\dot{I}^{2}$ and so, since $I^{3}$ is convex, are homotopic rel $\dot{I}^{2}$. Hence $\overline{h \circ \varphi_{0}}=\overline{h \circ \varphi_{1}}$ in $\rho_{2}$. But $\overline{h \circ \varphi_{0}}$ is the composite matrix given in the proposition, and $\overline{h \circ \varphi_{1}}=c_{1}$.

A map $f: X_{*} \rightarrow Y_{*}$ of triples clearly defines a morphism $\rho(f): \rho\left(X_{*}\right) \rightarrow \rho\left(Y_{*}\right)$ of double groupoids.

Proposition 6.4 If $f: X_{*} \rightarrow Y_{*}$ is a map of triples such that each of $f: X \rightarrow Y, f_{1}: X_{1} \rightarrow Y_{1}, f_{0}$ : $X_{0} \rightarrow Y_{0}$ are homotopy equivalences, then $\rho(f): \rho\left(X_{*}\right) \rightarrow \rho\left(Y_{*}\right)$ is an isomorphism.

Proof This is an immediate consequence of (10.11) of [89]. (In fact the maps $R_{i}\left(X_{*}\right) \rightarrow R_{i}\left(Y_{*}\right)$ are then homotopy equivalences, as it is not hard to deduce for $i=1,2$ from the cogluing theorem of [31].)

From the homotopy double groupoid $\rho\left(X_{*}\right)$ we obtain according to the procedure of the previous section a crossed module $\gamma\left(\rho\left(X_{*}\right)\right)$. 
Proposition 6.5 If $x \in X_{0}$, then the group $\gamma\left(\rho\left(X_{*}\right)\right)_{2}(x)$ may be identified with the group of homotopy classes rel

$$
J^{2}=(I \times \dot{I}) \cup(\{0\} \times I)
$$

of maps

$$
\left(I^{2},\{1\} \times I, J^{2}\right) \rightarrow\left(X, X_{1}, x\right),
$$

i.e. with the usual second relative homotopy group $\pi_{2}\left(X, X_{1}, x\right)$.

Proof Clearly there is a group surjection

$$
\phi: \pi_{2}\left(X, X_{1}, x\right) \rightarrow \gamma\left(\rho\left(X_{*}\right)\right)_{2}(x) .
$$

We have to prove $\phi$ is injective. Suppose then that $\phi(\bar{\alpha})=0$. Then there is an f-homotopy $h: \alpha \equiv 0$. We have to prove there is a homotopy of maps of triples $h^{\prime}: \alpha \simeq 0$ rel $J^{2}$, and this is done by using a filling argument to deform $h$ to a homotopy $h^{\prime}$ of the required type.

We write the elements of $I^{4}$ as $(r, s, t, u)$. We first define

$$
\begin{aligned}
& F(r, s, t, 0)=h(r, s, t) \\
& F(r, s, 0, u)=h(r, s, 0)=\alpha(r, s) \\
& F(r, s, 1, u)=x
\end{aligned}
$$

where the first says that $u=0$ gives the original homotopy, and the second and third say that $h_{0}=\alpha$ and $h_{1}$ are not altered in the homotopy of $h$. Next, the final homotopy, i.e. when $u=1$, must be rel $J^{2}$. This means that we define

$$
F(0, s, t, 1)=F(r, 0, t, 1)=F(r, 1, t, 1)=x .
$$

We need to define $F$ on seven of the 3 -faces of $I^{4}$ and so far it is defined on only three of them, as well as on three 2-faces. We start an extension process by defining $F$ to have value $x$ on the elements $(1,1, t, u)$, and find that $F$ is well defined on five of the faces of the 3-cube given by $s=1$. Hence $F$ may be extended over this 3 -cube. In a similar way we get $F$ defined on the 3 -cubes given in turn by $r=0$, next by $s=0$, and then by $r=1$. Now $F$ is defined on seven of the 3 -faces of $I^{4}$, and so extends over $I^{4}$. Then $F(r, s, t, 1)$ is the required homotopy, since by definition it is a homotopy of $\alpha$ rel $J^{2}$, and it does map the points $(1, s, t)$ into $X_{1}$, since all the faces of the 3 -cubes over which we extended were mapped into $X_{1}$.

On the face of it, this shows that the theory of the homotopy double groupoid $\rho\left(X_{*}\right)$ is equivalent to that of the classical second relative homotopy crossed module, and so the question can be fairly put: Why introduce a new version? The answer is the usual kind of answer, that sometimes the new version is useful for proving theorems. In particular, we are unable to prove directly in terms of crossed modules the version of the 2-dimensional Van Kampen theorem which gives a result in terms of the classical crossed modules. 
The reason for conceiving of the homotopy double groupoid was to find an algebraic gadget more appropriate than groups for giving an

algebraic inverse to subdivision.

This is the slogan underlying the work on higher dimensional Van Kampen Theorems. Subdividing a square into little squares has a convenient expression in terms of double groupoids, and much more inconvenient expressions, if they exist at all, in terms of crossed modules. The 2-dimensional Van Kampen Theorem was conceived first in terms of double groupoids, and it was only gradually that the link with crossed modules was realised. In the end, the aim of obtaining Whitehead's theorem on free crossed modules (Corollary 5.16) as a corollary was a key impetus to forming a definition of a homotopy double groupoid for a pointed pair of spaces, since that theorem involved a crossed module defined for such a pair of spaces.

Further, the connections on double groupoids which were found necessary to make precise the relations between crossed modules and double groupoids [50] turned out also to be exactly what was needed for the homotopy addition lemma and so allowed Lemma 6.10 in the proof of our 2dimensional Van Kampen Theorem in the next section. This lemma shows that a construction of an element of a particular double groupoid is independent of all the choices made. Applications of connections and thin structures for double categories are given in [106, 107].

The more restricted structure of a homotopy 2-groupoid associated to a triple has been indicated in [97], but again it is difficult to prove in a manner similar to that above the 2-dimensional Van Kampen Theorem using this structure.

Our theory gives in a sense an algebraic formulation of different ways which have been classically used in considering properties of relative homotopy groups. We find that the 2-dimensional double groupoid viewpoint is useful for understanding the theory and for proving theorems, while the crossed module viewpoint is useful for specific calculations, and because of its closer relation to chain complexes. The importance of the algebraic formulation of this equivalence is the equivalence between colimits, and in particular pushouts, in the two categories.

The reader will have noticed the common use of filling arguments in the above proofs. These arguments become even more essential in the proof of results for higher dimensions, as in [36].

In higher dimensions we have found it necessary to take the connections as basic structures, since it is relatively easy to define a cubical set with compositions and connections [35], but quite difficult, though necessary, to define inductively the notion of 'cube with commuting boundary'. The centrality of this and analogous problems, and their long occurrence in the history of algebraic topology, is one of the fascinations of higher dimensional algebra. 


\subsection{General statement and proof of the 2-dimensional Van Kampen theorem}

In this section we write $X_{*}$ for the triple $\left(X, X_{1}, X_{0}\right)$ of spaces. All double groupoids will be double groupoids with thin structure.

We say that $X_{*}$ is connected if the following conditions hold:

$(\ddagger)_{0}$ the maps $\pi_{0}\left(X_{0}\right) \rightarrow \pi_{0}\left(X_{1}\right)$ and $\pi_{0}\left(X_{0}\right) \rightarrow \pi_{0}(X)$ are surjective;

$(\ddagger)_{1}$ the morphism of groupoids $\pi_{1}\left(X_{1}, X_{0}\right) \rightarrow \pi_{1}\left(X, X_{0}\right)$ is piecewise surjective.

It may be shown that given $(\ddagger)_{0}$, the condition $(\ddagger)_{1}$ may be replaced by

$\left(f^{\prime}\right)_{1}$ For each $x \in X_{0}$, the homotopy fibre over $x$ of the inclusion $X_{1} \rightarrow X$ is path connected.

This explains the origin of the term 'connected' (see [44]).

Now suppose we are given a cover $\mathcal{U}=\left\{U^{\lambda}\right\}_{\lambda \in \Lambda}$ of $X$ such that the interiors of the sets of $\mathcal{U}$ cover $X$. For each $\nu \in \Lambda^{n}$ we write

$$
U^{\nu}=U^{\nu_{1}} \cap \ldots \cap U^{\nu_{n}}
$$

$U_{i}^{\nu}=U^{\nu} \cap X_{i}$, and $U_{*}^{\nu}=\left(U^{\nu}, U_{1}^{\nu}, U_{0}^{\nu}\right)$. So the homotopy double groupoids in the following $\rho$-sequence of the cover are well-defined:

$$
\bigsqcup_{\nu \in \Lambda^{2}} \rho\left(U_{*}^{\nu}\right) \underset{b}{\stackrel{a}{\longrightarrow}} \bigsqcup_{\lambda \in \Lambda} \rho\left(U_{*}^{\lambda}\right) \stackrel{c}{\longrightarrow} \rho\left(X_{*}\right)
$$

Here $\sqcup$ denotes disjoint union, which is the coproduct in the category of double groupoids. It is an advantage of the approach using a set of base points that the coproduct in this category is so simple to describe. The morphisms $a, b$ are determined by the inclusions

$$
a_{\nu}: U^{\lambda} \cap U^{\mu} \rightarrow U^{\lambda}, b_{\nu}: U^{\lambda} \cap U^{\mu} \rightarrow U^{\mu}
$$

for each $\nu=(\lambda, \mu) \in \Lambda^{2}$, and $c$ is determined by the inclusion $c_{\lambda}: U^{\lambda} \rightarrow X$ for each $\lambda \in \Lambda$.

Theorem 6.6 [34, Theorem B] Assume that for every finite intersection $U^{\nu}$ of elements of $\mathcal{U}$ the triple $U_{*}^{\nu}$ is connected. Then

(C) the triple $X_{*}$ is connected, and

(I) in the above $\rho$-sequence of the cover, $c$ is the coequaliser of $a, b$ in the category of double groupoids.

The proof will take several stages. The first step is the following one. 
Lemma 6.7 Let $\alpha \in R_{2}\left(X_{*}\right)$ and let $\alpha=\left[\alpha_{i j}\right]$ be a subdivision of $\alpha$ such that each $\alpha_{i j}$ lies in some $U^{i j}$, a finite intersection of elements of $U$. Then there is an f-homotopy $h: \alpha \equiv \theta$, with $\theta \in R_{2}\left(X_{*}\right)$, such that, in the subdivision $h=\left[h_{i j}\right]$ determined by that of $\alpha$, each homotopy $h_{i j}: \alpha_{i j} \simeq \theta_{i j}$ satisfies:

(i) $h_{i j}$ lies in $U^{i j}$;

(ii) $\theta_{i j}$ belongs to $R_{2}\left(X_{*}\right)$;

(iii) if a vertex $v$ of the domain of $\alpha_{i j}$ is mapped into $X_{0}$, then $h$ is constant on $v$;

(iv) if for $r=0,1$ a cell $e$ of the domain of $\alpha_{i j}$ is mapped by $\alpha$ into $X_{r}$, then $e \times I$ is mapped by $h$ into $X_{r}$, and hence $\theta(e)$ is contained in $X_{r}$.

Proof Let $K$ be the cell-structure on $I^{2}$ determined by the subdivision $\alpha=\left[\alpha_{i j}\right]$. Let $L_{m}=$ $K^{m} \times I \cup K \times\{0\}$ and $X_{2}=X$. We construct maps $h_{m}: L_{m} \rightarrow X_{2}$, for $m=0,1,2$, such that $h_{m}$ extends $h_{m-1}$, where $h_{-1}=\alpha$. Further we construct $h_{m}$ to satisfy the following conditions, for each m-cell $\sigma$ of $K$ :

$\left(a_{m}\right): h_{m} \mid \sigma \times\{1\}$ is an element of $R_{m}\left(X_{*}\right)$;

$\left(b_{m}\right)$ : if $\alpha$ maps $\sigma$ into $X_{r}$, then $h_{m}(\sigma \times I) \subset X_{r}$;

$\left(c_{m}\right)$ : if $\sigma$ is contained in the domain of $\alpha_{i j}$, then $h_{m}(\sigma \times I) \subset U^{i j}$.

The construction of $h_{m}$ from $h_{m-1}$ is as follows. We consider an $m$-cell $\sigma$ of $K$, and let $r$ be the smallest integer such that $\alpha$ maps $\sigma$ into $X_{r}$. If $r \leq m$, then $h_{m-1}$ can be extended to $h_{m}$ on $\sigma \times I$ by means of a retraction $\alpha \times I \rightarrow \sigma \times\{0\} \cup \dot{\sigma} \times I$. If $r>m$ let $U^{\sigma}$ be the intersection of all the sets $U^{i j}$ such that $\sigma$ is contained in the domain of $\alpha_{i j}$. The restriction of $h_{m-1}$ to the pair $(\sigma \times\{0\} \cup \dot{\sigma} \times I, \dot{\sigma} \times I)$ determines an element of $\pi_{m}\left(U_{r}^{\sigma}, U_{m-1}^{\sigma}\right)$. (Here $m \leq 1$ and $U_{-1}^{\sigma}$ is taken to be $\emptyset$.) By $(\ddagger)_{m}, h_{m-1}$ extends to $h_{m}$ on $\sigma \times I$ mapping into $U_{r}^{\sigma}$ and such that $\sigma \times\{1\}$ is mapped into $U_{m}^{\sigma}$.

The connectivity result (C) is immediate from this lemma, particularly (iv), applied to doubly degenerate or to degenerate squares representing elements of an appropriate $\pi_{0}$ or $\pi_{1}$.

We next prove the coequaliser result.

Suppose we are given a morphism

$$
f^{\prime}: \coprod_{\lambda \in \Lambda} \pi\left(U_{*}^{\lambda}\right) \rightarrow G
$$

of double groupoids such that $f^{\prime} \circ a=f^{\prime} \circ b$. We have to show that there is a unique morphism $f: \rho\left(X_{*}\right) \rightarrow G$ of double groupoids such that $f \circ c=f^{\prime}$.

Let $p_{\lambda}: R\left(U_{*}^{\lambda}\right) \rightarrow \rho\left(U_{*}^{\lambda}\right)$ be the projection and let $F_{\lambda}=f^{\prime} \circ p_{\lambda}: R\left(U_{*}^{\lambda}\right) \rightarrow G$. We first define $f$ on $\rho_{2}\left(X_{*}\right)$ and to this end first construct $F: R_{2}\left(X_{*}\right) \rightarrow G$. 
Suppose that $\theta$ in $R_{2}\left(X_{*}\right)$ is such that $\theta$ in $R_{2}\left(X_{*}\right)$ is such that $\theta$ lies in some set $U^{\lambda}$ of $U$. Then $\theta$ determines uniquely an element $\theta^{\lambda}$ of $R_{2}\left(U_{*}^{\lambda}\right)$ and the rule $f^{\prime} \circ a=f^{\prime} \circ b$ implies that

$$
F(\theta)=F_{\lambda}\left(\theta^{\lambda}\right)
$$

is determined by $\theta$.

Suppose we are given a subdivision $\theta=\left[\theta_{i j}\right]$ of an element $\theta$ in $R_{2}\left(X_{*}\right)$ such that each $\theta_{i j}$ is in $R_{2}\left(X_{*}\right)$ and also lies in some $U^{\nu}$, for $\nu \in \Lambda^{n}$. Then $\theta_{i j}$ also lies in some $U^{\lambda}$, with $\lambda \in \Lambda$, and since the composite $\left[\theta_{i j}\right]$ is defined it is easy to check, again using $f^{\prime} \circ a=f^{\prime} \circ b$, that the elements $F\left(\theta_{i j}\right)$ compose in $G$ to give an element $g=\left[F\left(\theta_{i j}\right)\right]$, which we write as $F(\alpha)$ although a priori it depends on the subdivision chosen.

We next wish to construct $F(\alpha)$ for an arbitrary element $\alpha$ of $F_{2}\left(X_{*}\right)$. This construction is based on Lemma 6.7.

Corollary 6.8 Let $\alpha \in R_{2}\left(X_{*}\right)$. Then there is an f-homotopy $h: \alpha \equiv \theta$ such that $F(\theta)$ is defined in $G_{2}$.

Proof Choose a subdivision $\alpha=\left[\alpha_{i j}\right]$ such that each $\alpha_{i j}$ lies in some set $U^{i j}$ of $\mathrm{U}$. Then apply Lemma 6.7.

This element $F(\theta)$ of the corollary we write $F\left(\alpha,\left(h_{i j}\right)\right)$ and prove first that it depends only on $\alpha$. Accordingly, let $h^{\prime}: \alpha \equiv \theta^{\prime}$ be an alternative f-homotopy satisfying the conditions of Lemma 6.7 with respect to a subdivision $\alpha=\left[\alpha_{k l}^{\prime}\right]$ in which each $\alpha_{k l}^{\prime}$ lies in some set $V^{k l}$ of U. Since any two subdivisions have a common refinement we may assume, without loss of generality, that $\left[\alpha_{k l}^{\prime}\right]$ is a refinement of $\left[\alpha_{i j}\right]$.

For each $(k l)$, let $W^{k l}=V^{k l} \cap U^{i j}$ where $U^{i j}$ is such that $\alpha_{k l}^{\prime}$ is a part of $\alpha_{i j}$. By Lemma 1 there is an f-homotopy $h^{\dagger}=\left[h_{k l}^{\dagger}\right]$ from $\alpha$ to $\theta^{\dagger}$ such that each $h_{k l}^{\dagger}$ lies in $W^{k l}$. The f-homotopy $H=\bar{h}^{\prime} h^{\dagger}: \theta^{\prime} \equiv \theta^{\dagger}$ (where $\bar{h}^{\prime}$ is the reverse of $h^{\prime}$ ) has the subdivision $H=\left[H_{k l}\right]$ where $H_{k l}: \theta^{\prime} \simeq \theta_{k l}^{\dagger}$ and $H_{k l}$ lies in $V^{k l}$.

Let $\theta_{i j}^{*}$ be the composite of those $\theta_{k l}^{\dagger}$ such that $\alpha_{k l}^{\prime}$ is a part of $\alpha_{i j}$. Then we also have a subdivision $h^{\dagger}=\left[h_{i j}^{*}\right]$, where $h_{i j}^{*}: \alpha_{i j} \simeq \theta_{i j}^{*}$ lies in $U_{i j}$. So $H^{*}=\bar{h}^{\dagger} h: \theta^{\dagger} \equiv \theta$ is an f-homotopy with subdivision $H^{*}=\left[H_{i j}^{*}\right]$, where $H_{i j}^{*}: \theta_{i j}^{*} \simeq \theta_{i j}$ is a homotopy lying in $U^{i j}$.

It will follow from Lemma 6.10 below that $\left[F\left(\theta_{k l}^{\prime}\right]=\left[F\left(\theta_{k l}^{\dagger}\right)\right]\right.$ and $\left[F\left(\theta_{i j}^{*}\right)\right]=\left[F\left(\theta_{i j}\right)\right]$. However $\left[F\left(\theta_{i j}^{*}\right]=\left[F\left(\theta_{k l}^{\dagger}\right)\right]\right.$, since the latter is a refinement of the former. Hence $\left[F\left(\theta_{k l}^{\prime}\right]=\left[F\left(\theta_{i j}\right)\right]\right.$ and so $F\left(\alpha,\left(h_{i j}\right)\right)$ depends only on $\alpha$.

Lemma 6.9 Let $\theta, \theta^{*} \in R_{2}$ and suppose we are given an f-homotopy $H: \theta \equiv \theta^{*}$. Let $H=\left[H_{i j}\right]$ be a subdivision such that each $H_{i j}$ lies in some set $U^{i j}$ of $U$. Let $\theta=\left[\theta_{i j}\right], \theta^{*}=\left[\theta_{i j}^{*}\right]$ be the subdivisions of $\theta, \theta^{*}$ induced by that of $H$, and suppose that $\theta_{i j}, \theta_{i j}^{*}$ are in $R_{2}$ for all $(i, j)$. Then $H$ is homotopic rel end maps to an f-homotopy $\hat{H}: \theta \equiv \theta^{*}$ such that for all $i, j$, 
(i) $\hat{H}_{i j}$ has its edges in $X_{1}$,

(ii) $\hat{H}_{i j}$ lies in $U^{i j}$.

Proof The proof is similar to that of Lemma 6.7. The subdivision $\theta=\left[\theta_{i j}\right]$ induces a cell-structure $K$ on $I^{2}$, and the homotopy $H \simeq \hat{H}$ is constructed on $K^{m} \times I \times I \cup K \times \dot{I} \times I \cup K \times I \times\{0\}$ by induction on $m$.

NOTE. We do not claim that $\hat{H}_{i j}$ is an f-homotopy $\theta_{i j} \equiv \theta_{i j}^{*}$.

Lemma 6.10 Let $\theta, \theta^{*}, H,\left(H_{i j}\right)$ be as in Lemma 6.9. Then in $G_{2}$,

$$
\left[F\left(\theta_{i j}\right)\right]=\left[F\left(\theta_{i j}^{*}\right)\right]
$$

Proof We replace $H$ by the $\hat{H}: \theta \equiv \theta^{*}$ given by Lemma 6.9. Let $F\left(\theta_{i j}\right)=c_{i j}, F\left(\theta_{i j}^{*}\right)=c_{i j}^{*}$. Since $\hat{H}_{i j}$ has its edges in $X_{1}$ and vertices in $X_{0}$, the homotopy addition lemma (Proposition 6.3) gives, on applying $F$, a relation in $G_{2}$ of the form

$$
c_{i j}^{*}=\left[\begin{array}{ccc}
\Gamma & a_{i-1, j}^{-1} & \neg \\
-b_{i, j-1} & c_{i j} & b_{i j} \\
\llcorner & a_{i j} & \lrcorner
\end{array}\right]
$$

where the $a^{\prime} s$ and $b^{\prime} a$ are images in $G_{2}$ of certain faces of the $\hat{H}_{i j}$.

The interchange law for $G$ allows us to refine the subdivision $c^{*}=\left[c_{i j}^{*}\right]$ by the substitution $(\dagger)$ and to compose the parts in any convenient fashion. By cancellation of pairs $b_{i j},-b_{i j}$ and $a_{i j}, a_{i j}^{-1}$, and by composing thin elements, including $0^{\prime} s$ and $1^{\prime} s$, we can obtain a new subdivision of $c^{*}$ of the form

$$
c^{*}=\left[\begin{array}{ccc}
\ulcorner & a_{0}^{-1} & \neg \\
-b_{0} & c & b_{1} \\
\llcorner & a_{1} & \lrcorner
\end{array}\right]
$$

where $c=\left[c_{i j}\right]$ and the elements $a_{i}, b_{i}$ are composites in $G_{2}$ of the images of squares lying on the boundary of $\hat{H}$. Since $\hat{H}$ is an f-homotopy, these squares are in $X_{1}$ and so the $a_{i}, b_{i}$ are thin. Since the homotopies are rel vertices, the corner elements in (31) are $\odot$. It now follows that the $a_{i}$ are $1^{\prime} s$ and the $b_{i}$ are $0^{\prime} s$, and therefore $c^{*}=c$.

With the proof of Lemma 6.10 we have completed the proof that $F\left(\alpha,\left(h_{i j}\right)\right)$ depends only on $\alpha$.

Lemma $6.11 F\left(\alpha,\left(h_{i j}\right)\right)$ depends only on the class of $\alpha$ in $\rho_{2}$. 
Proof Let $K: \alpha \equiv \alpha^{\prime}$ be an f-homotopy. Then there is an $(m \times n \times p)$-subdivision $K=\left[K_{i j k}\right]$ such that each $K_{i j k}$ lies in some set of $\mathrm{U}$, say $U^{i j k}$. Let $\alpha=\left[\alpha_{i j}\right], \alpha^{\prime}=\left[\alpha_{i j}^{\prime}\right]$ be the induced subdivisions of $\alpha, \alpha^{\prime}$. A simple induction on $p$ reduces us to the case where $p=1$, and so we may assume that the subdivision of $K$ has a single layer $K=\left[K_{i j}\right]$, each $K_{i j}$ being a homotopy $\alpha_{i j} \simeq \alpha_{i j}^{\prime}$ lying in $U^{i j}$. Then we choose $h: \alpha \equiv \theta, h^{\prime}: \alpha^{\prime} \equiv \theta^{\prime}$ as in Lemma 6.7. Let $H$ be the composite homotopy $\bar{h} k h^{\prime}: \theta \equiv \theta^{\prime}$. Then by Lemma 6.10, $\left[F\left(\theta_{i j}\right)\right]=\left[F\left(\theta_{i j}^{\prime}\right)\right]$. Hence $F\left(\alpha,\left(h_{i j}\right)\right)=F\left(\alpha^{\prime},\left(h_{i^{\prime} j^{\prime}}^{\prime}\right)\right)$.

We have now proved that there is a well-defined map $f: \rho\left(X_{*}\right) \rightarrow G_{2}$, given by $f(\bar{\alpha})=$ $F\left(\alpha,\left(h_{i j}\right)\right)$, and which satisfies $f \circ c=f^{\prime}$ at least on 2-dimensional elements of $\rho$.

The remainder of the proof of (I) is straightforward. It is easy to check that $f$ preserves addition and composition of squares, and it follows from (iii) of Lemma 6.7 that $f$ preserves thin elements.

It is now easy to extend $f$ to a morphism $f: \rho\left(X_{*}\right) \rightarrow G$ of double groupoids, since the 1- and 0 -dimensional parts of a double groupoid determine degenerate 2-dimensional parts. Clearly this $f$ satisfies $f \circ c=f^{\prime}$ and is the only such morphism.

This completes the proof of Theorem 6.6. Of especial interest (but not essentially easier to prove) is the case of Theorem 6.6 in which the cover $\mathcal{U}$ has only two elements; in this case Theorem 6.6 gives a push-out of double groupoids. In the applications below we shall consider only pathconnected spaces and assume that $Z=\{\xi\}$ is a singleton. Taking $\xi$ as base point, the double groupoids can then be interpreted as crossed modules of groups to give the 2-dimensional analogue of the Seifert-van Kampen theorem given as Theorem 4.1 earlier. We do not know how to prove that theorem without using groupoids in some form. A higher dimensional form of this proof and theorem is given in [36] and the theorem is stated later as Theorem 7.6.

Proof of Theorem 4.1 In the case where $(X, A)$ is a based pair with base point $\xi, \rho(X, A, \xi)$ is abbreviated to $\rho(X, A)$. That we obtain a pushout of crossed modules under Hypothesis $\mathrm{A}$ is simply a special case of Theorem 6.6, together with Proposition 6.5, which gives the equivalence between double groupoids and crossed modules.

The corresponding result under Hypothesis B follows from that under Hypothesis A by standard techniques using mapping cylinders (see a similar proof in $[16,8.4 .2]$ ).

Remark 6.12 An examination of the proof of Theorem 6.6 shows that condition $(\ddagger)_{m}$ is required only for 8-fold intersections of elements of $\mathcal{U}$. However, it has been shown by Razak-Salleh [103] that in fact one need only assume $(\ddagger)_{0}$ for 4 -fold intersections and $(\ddagger)_{1}$ for 3 -fold intersections. Further, these conditions are best possible.

Remark 6.13 Theorem 6.6 contains 1-dimensional information which includes most known results expressing the fundamental group of a space in terms of an open cover, but it does not assume that the spaces of the cover or their intersections are path-connected.

An alternative proof of Theorem 4.1 is given in [44] using cat ${ }^{1}$-groups, which are equivalent to group objects in the category of groupoids, and to reduced crossed modules [51]. This proof uses 
many more results from algebraic topology. On the other hand, this proof extends to cat $^{n}$-groups [44] and has more powerful applications than Theorem 7.6. A survey of the results, and some new ones, is given in [21]. Some remarks on this are given in the final section 8. 


\section{$7 \quad$ The category of crossed complexes}

The definition of a crossed complex generalises to the case of a set of base points definitions given by Blakers [11] (under the term 'group system') and Whitehead [118], under the term 'homotopy system' (except that he restricted also to the free case). We recall this general definition from [35]. Of course the case for not restricting to the reduced case, i.e. of a single base point, is analogous to that for not restricting to reduced $C W$-complexes.

A crossed complex $C$ (of groupoids) is a sequence of morphisms of groupoids over $C_{0}$

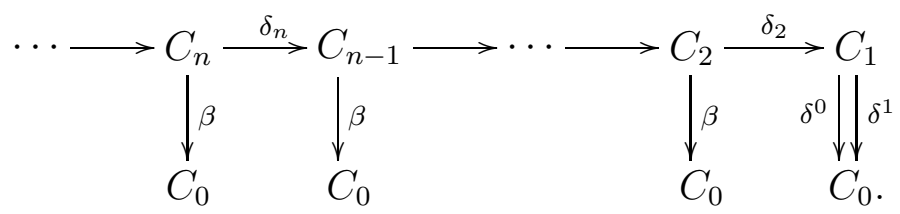

Here $\left\{C_{n}\right\}_{n} \geqslant 2$ is a family of groups with base point map $\beta$, and $\delta^{0}, \delta^{1}$ are the source and targets for the groupoid $C_{1}$. We further require given an operation of the groupoid $C_{1}$ on each family of groups $C_{n}$ for $n \geqslant 2$ such that:

(i) each $\delta_{n}$ is a morphism over the identity on $C_{0}$;

(ii) $C_{2} \rightarrow C_{1}$ is a crossed module over $C_{1}$;

(iii) $C_{n}$ is a $C_{1}$-module for $n \geqslant 3$;

(iv) $\delta: C_{n} \rightarrow C_{n-1}$ is an operator morphism for $n \geqslant 3$;

(v) $\delta \delta: C_{n} \rightarrow C_{n-2}$ is trivial for $n \geqslant 3$;

(vi) $\delta C_{2}$ acts trivially on $C_{n}$ for $n \geqslant 3$.

Because of axiom (iii) we shall write the composition in $C_{n}$ additively for $n \geqslant 3$, but we will use multiplicative notation in dimensions 1 and 2 .

Let $C$ be a crossed complex. Its fundamental groupoid $\pi_{1} C$ is the quotient of the groupoid $C_{1}$ by the normal, totally disconnected subgroupoid $\delta C_{2}$. The rules for a crossed complex give $C_{n}$, for $n \geqslant 3$, the induced structure of $\pi_{1} C$-module.

The crossed complex $C$ is reduced if $C_{0}$ is a singleton, so that all the groupoids $C_{n}, n \geqslant 1$ are groups. This was the case considered in [11, 118] and many other sources.

A morphism $f: C \rightarrow D$ of crossed complexes is a family of groupoid morphisms $f_{n}: C_{n} \rightarrow$ $D_{n}(n \geqslant 0)$ which preserves all the structure. This defines the category Crs of crossed complexes. The fundamental groupoid now gives a functor $\pi_{1}: \mathrm{Crs} \rightarrow \mathrm{Gpd}$. This functor is left adjoint to the functor $i:$ Gpd $\rightarrow$ Crs where for a groupoid $G$ the crossed complex $i G$ agrees with $G$ in dimensions 0 and 1 , and is otherwise trivial. 
An $m$-truncated crossed complex $C$ consists of all the structure defined above but only for $n \leqslant m$. In particular, an $m$-truncated crossed complex is for $m=0,1,2$ simply a set, a groupoid, and a crossed module respectively.

In order to give the basic geometric example of a crossed complex we first define a filtered space $X_{*}$. By this we mean a topological space $X_{\infty}$ and an increasing sequence of subspaces

$$
X_{*}: X_{0} \subseteq X_{1} \subseteq \cdots \subseteq X_{n} \subseteq \cdots \subseteq X_{\infty}
$$

A map $f: X_{*} \rightarrow Y_{*}$ of filtered spaces consists of a map $f: X_{\infty} \rightarrow Y_{\infty}$ of spaces such that for all $i \geqslant 0, f\left(X_{i}\right) \subseteq Y_{i}$. This defines the category FTop of filtered spaces and their maps.

We now define the fundamental, or homotopy, crossed complex functor

$$
\pi: \text { FTop } \rightarrow \text { Crs. }
$$

If $C=\pi\left(X_{*}\right)$, then $C_{0}=X_{0}$, and $C_{1}$ is the fundamental groupoid $\pi_{1}\left(X_{1}, X_{0}\right)$. For $n \geqslant 2, C_{n}=\pi_{n} X_{*}$ is the family of relative homotopy groups $\pi_{n}\left(X_{n}, X_{n-1}, p\right)$ for all $p \in X_{0}$. These come equipped with the standard operations of $\pi_{1} X_{*}$ on $\pi_{n} X_{*}$ and boundary maps $\delta: \pi_{n} X_{*} \rightarrow \pi_{n-1} X_{*}$, namely the boundary of the homotopy exact sequence of the triple $\left(X_{n}, X_{n-1}, X_{n-2}\right)$. The axioms for crossed complexes are in fact those universally satisfied for this example, but this cannot be proved at this stage.

We can now develop the following situation, discussed in more detail in later sections:

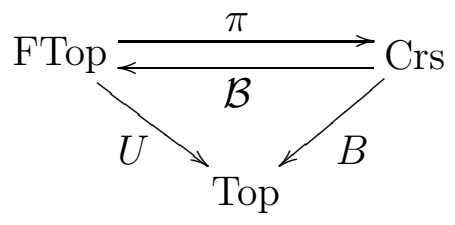

Here the functor $\pi$ has already been defined. The functor $U$ is the forgetful functor $X_{*} \mapsto X_{\infty}$. The functor $\mathcal{B}$ is the full classifying space functor and the functor $B=U \circ \mathcal{B}$ is the classifying space functor.

These functors have the following properties:

7.1 The functor $\pi$ satisfies a Generalised Van Kampen Theorem.

7.2 The composite functor $\pi \circ \mathcal{B}$ is naturally equivalent to the identity functor.

7.3 There is a natural transformation $1 \rightarrow \mathcal{B} \circ \pi$ in the weak homotopy category of FTop, with convenient properties. 
The first and third of these statements are vague. All three statements relate topology and algebra. The functor $\pi$ goes from topology to algebra and the classifying space functors go in the reverse direction. It is important that the functor $\pi$ is from structured spaces to algebra. This was not the original expectation of this theory, but this is how it has conveniently worked out.

The first statement allows for the computation of $\pi$ to some extent. The amount of this extent can only be judged when the proper statement is given, but here we can say that the resulting computations allow one to get started.

The second statement relates closely the structure on the spaces and the algebra under consideration.

The final statement is more subtle, but it states essentially that the algebra captures a slice of the homotopy structure of the original filtered space.

These properties are a kind of paradigm for relating topology and algebra. When these properties hold, we have a tool which can directly relate topology and algebra. If some of these properties are dropped, then we have more trouble in developing results.

For example, simplicial groups form an algebraic category strongly used in algebraic topology. They could be considered as a kind of 'higher dimensional group'. However, we do not at present have a functor (topological data) $\rightarrow$ (simplicial groups) satisfying a Van Kampen Theorem, and so simplicial groups cannot be used in the kind of way described here for these other algebraic models. Some suggestions for developments on these lines are given in [64].

In particular, the concept of induced crossed module and its application to specific calculation of homotopy 2-types has not so far been directly linked with work on simplicial groups. On the other hand, simplicial groups are used crucially in the proof of the Generalised Van Kampen Theorem for cat $^{n}$-groups by Brown and Loday in [44]. This suggests that there is much more work to be done on developing these links, and exploiting the two areas together. This is the subject of current work, for example $[55,101,112]$.

\subsection{The Generalised Van Kampen Theorem for Crossed Complexes}

Definition 7.4 A filtered space $X_{*}$ is called connected if the following conditions $\phi(X, m)$ hold for each $m \geqslant 0$ :

$\phi(X, 0):$ If $j>0$, the map $\pi_{0} X_{0} \rightarrow \pi_{0} X_{j}$, induced by inclusion, is surjective

$\phi(X, m),(m \geqslant 1):$ If $j>m$ and $\nu \in X_{0}$, then the map

$$
\pi_{m}\left(X_{m}, X_{m-1}, \nu\right) \rightarrow \pi_{m}\left(X_{j}, X_{m-1}, \nu\right)
$$

induced by inclusion, is surjective.

The following result gives another useful formulation of this condition. We omit the proof. 
Proposition 7.5 A filtered space $X$ is connected if and only if for all $n>0$ the induced map $\pi_{0} X_{0} \rightarrow \pi_{0} X_{n}$ is surjective and for all $r>n>0$ and $\nu \in X_{0}, \pi_{n}\left(X_{r}, X_{n}, \nu\right)=0$.

Suppose for the rest of this section that $X_{*}$ is a filtered space. Let $X=X_{\infty}$.

We suppose given a cover $\mathcal{U}=\left\{U^{\lambda}\right\}_{\lambda \in \Lambda}$ of $X$ such that the interiors of the sets of $\mathcal{U}$ cover $X$. For each $\zeta \in \Lambda^{n}$ we set

$$
U^{\zeta}=U^{\zeta_{1}} \cap \cdots \cap U^{\zeta_{n}}, U_{i}^{\zeta}=U^{\zeta} \cap X_{i}
$$

Then $U_{0}^{\zeta} \subseteq U_{1}^{\zeta} \subseteq \cdots$ is called the induced filtration $U_{*}^{\zeta}$ of $U^{\zeta}$. Consider the following $\pi$-diagram of the cover:

$$
\bigsqcup_{\zeta \in \Lambda^{2}} \pi U_{*}^{\zeta} \stackrel{a}{\underset{b}{\longrightarrow}} \bigsqcup_{\lambda \in \Lambda} \pi U_{*}^{\lambda} \stackrel{c}{\longrightarrow} \pi X_{*}
$$

Here $\sqcup$ denotes disjoint union (which is the same as coproduct in the category of crossed complexes); $a, b$ are determined by the inclusions $a_{\zeta}: U^{\lambda} \cap U^{\mu} \rightarrow U^{\lambda}, b_{\zeta}: U^{\lambda} \cap U^{\mu} \rightarrow U^{\mu}$ for each $\zeta=(\lambda, \mu) \in \Lambda^{2}$; and $c$ is determined by the inclusions $c_{\lambda}: U^{\lambda} \rightarrow X$.

Theorem 7.6 (The coequaliser theorem for crossed complexes: Brown and Higgins [36]) Suppose that for every finite intersection $U^{\zeta}$ of elements of $\mathcal{U}$ the induced filtration $U_{*}^{\zeta}$ is connected. Then

(C) $X_{*}$ is connected, and

(I) in the above $\pi$-diagram of the cover, $c$ is the coequaliser of $a, b$ in the category of crossed complexes.

A version of the Van Kampen Theorem tied to dimension $n$ can now be formulated as follows. First, we say the pair $(X, A)$ is $(n-1)$-connected if $A$ and $X$ are path connected, and the induced map of homotopy groups $\pi_{i}(A, x) \rightarrow \pi_{i}(X, x)$ is bijective for $1 \leqslant i<n-1$ and surjective for $i=n-1$. This is equivalent to saying that the homotopy fibre over $x$ of the inclusion $A \rightarrow X$ is $(n-1)$-connected, or that the relative homotopy group (or set for $i=1$ ) $\pi_{i}(X, A)=0$ for $1 \leqslant i \leqslant n-1$.

If $G$ is a group and $M$ is a $G$-module, then we call the pair $(M, G)$ simply a module. These modules form the objects of a category Mod, whose morphisms $(\phi, \psi):(M, G) \rightarrow(N, H)$ are pairs of morphisms of groups $\phi: M \rightarrow N, \psi: G \rightarrow H$ which preserve the action, i.e. $\phi\left(m^{g}\right)=(\phi m)^{\psi g}$ for all $m \in M, g \in G$. This category is complete and cocomplete.

If $(X, A)$ is a pointed pair of spaces, we write $\Pi_{n}(X, A)$ for the module $\left(\pi_{n}(X, A), \pi_{1}(A)\right)$ consisting of the group $\pi_{1}(A)$ and the $\pi_{1}(A)$-module $\pi_{n}(X, A)$. This gives a functor from the category of pairs of pointed spaces to Mod.

The following two results now follow by specialisation. 
Theorem 7.7 (Brown and Higgins [36]) Suppose that the commutative diagram of based pairs of spaces

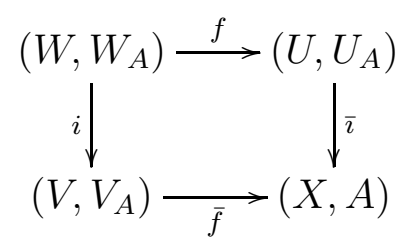

satisfies one of the two following hypotheses:

HYPOTHESIS A: the maps $i, f, \bar{\imath}, \bar{f}$ are inclusions of subspaces, $X$ is the union of the interiors of the sets $U$ and $V, W=U \cap V$, and $V_{A}=A \cap V, U_{A}=A \cap U, W_{A}=A \cap W$;

HYPOTHESIS B: the maps $i: W \rightarrow V, i_{A}: W_{A} \rightarrow V_{A}$ are closed cofibrations, $W_{A}=W \cap V_{A}$, and $X, A$ are the adjunction spaces $U \cup_{f} V, U_{A} \cup_{f_{A}} V_{A}$.

Let $n \geqslant 3$. Suppose further that all the pairs $\left(U, U_{A}\right),\left(V, V_{A}\right),\left(W, W_{A}\right)$ are $(n-1)$-connected. Then:

(C) The pair $(X, A)$ is $(n-1)$-connected.

(I) The following diagram induced by inclusions

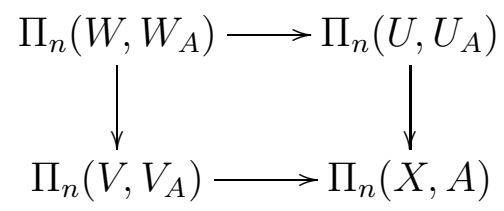

is a pushout of modules.

In a similar manner to the crossed module case $n=2$ we obtain:

Theorem 7.8 (Brown and Higgins [36]) Suppose that the commutative square

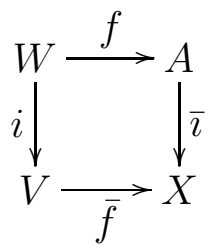

of based spaces satisfies one of the two following hypotheses:

HYPOTHESIS A: the maps $i, f, \bar{\imath}, \bar{f}$ are inclusions of subspaces, $W=V \cap A$, and $X$ is the union of the interiors of $V$ and $A$; 
HYPOTHESIS B: the map $i$ is a closed cofibration and $X$ is the adjunction space $A \cup_{f} V$.

Let $n \geqslant 3$. Suppose further that $(V, W)$ is $(n-1)$-connected and $A$ is path connected. Then:

(C) $(X, A)$ is $(n-1)$-connected;

(I) $\pi_{n}(X, A)$ is the $\pi_{1}(A)$-module induced from $\pi_{n}(V, W)$ by the morphism $f_{*}: \pi_{1}(W) \rightarrow \pi_{1}(A)$.

It is not clear that this theorem is essentially any easier to prove than Theorem 7.6.

The notion of induced module is much more standard than that of induced crossed module and may be found for example in books on representation theory although usually only for the case of inducing from a module over a subgroup of a group. It is a key part of the general subject of Mackey functors. One standard description for the $Q$-module $\iota_{*} M$ induced from a $P$-module $M$ by a morphism $\iota: P \rightarrow Q$ of groups is $\iota_{*} M \cong M \otimes_{\mathrm{Z} P} \mathrm{Z} Q$ where $P$ acts on $Q$ on the left via $\iota$. This suggest a possible notation of $M \otimes_{P} Q$ for the crossed module induced from a crossed module $M \rightarrow P$ by a subgroup inclusion $P \rightarrow Q$.

We now obtain as a Corollary one of the basic theorems of homotopy theory.

Corollary 7.9 (Relative Hurewicz Theorem) Let $n \geqslant 3$. Let $(V, W)$ be an $(n-1)$-connected pair of connected spaces, and let $X=V \cup C W$. Then $X$ is $(n-1)$-connected and $\pi_{n}(X)$ is isomorphic to the group $\pi_{n}(V, W)$ factored by the action of $\pi_{1}(W)$.

Notice that again we have a Relative Hurewicz Theorem without any mention of homology. The usual version follows from this result and the absolute Hurewicz Theorem, since $X$ is $(n-1)$ connected implies that $\pi_{n}(X)$ is isomorphic to $H_{n}(X)$, which itself is simply $H_{n}(V, W)$.

This deduction of the Relative Hurewicz Theorem from a Generalised Van Kampen Theorem was later modelled in the formulation and proof of an $n$-cubical Hurewicz Theorem [45, 20], giving it as a form of abelianisation, and interpreting this for triad groups. The interpretation in terms of the passage from $n$-adic homotopy to $n$-adic homology requires the definition of crossed $n$-cube of groups [71], since this tells you precisely what structure has to be factored in this passage. Another feature of the proof of the $n$-adic Hurewicz Theorem is that it requires the notion of $n$-pushout, i.e. an $n$-cubical version of a pushout square, and that it follows from an $n$-cubical version of excision.

Another useful result is the following higher dimensional version of Whitehead's Theorem.

Theorem 7.10 Let $n \geqslant 3$. Let $X=A \cup\left\{e_{\lambda}^{n}\right\}_{\lambda \in \Lambda}$ be obtained from the connected space A by attaching $n$-cells. Then the $n$ 'th relative homotopy group $\pi_{n}(X, A)$ may be described as the free $\pi_{1}(A)$-module on the $n$-cells.

However this result is not as impressive as the 2-dimensional version, since it may be and usually is deduced from the Relative Hurewicz Theorem, which can be proved separately. On the other hand, this mode of deduction is used in $[45,69]$ to obtain free crossed squares arising in topological situations. 
Corollary 7.11 If $X_{*}$ is the filtered space of the skeletons of a $C W$-complex, then the crossed complex $\pi X_{*}$ is a free crossed complex.

Remark 7.12 The proof of Theorem 7.6 follows a line analogous to the proof of Theorem 4.1. However, it is much harder work establishing the necessary algebra and defining the necessary higher homotopy groupoids. The proof itself needs some new ideas to make the intuition work. For these reasons, we omit further exposition.

An account of covering morphisms of crossed complexes is given in [49]. This enables one to give an $n$-dimensional version of the Cayley graph for a presentation of a group, and this leads to a kind of algorithm for calculating identities among relations.

\subsection{The nerve and classifying space of a crossed complex}

We let $\Delta^{n}$ be the cell complex of the standard $n$-simplex, with its skeletal filtration. The crossed complex $\pi \Delta^{n}$ is then written $\pi[n]$. The nerve $N C$ of a crossed complex $C$ is defined to be the simplicial set given in dimension $n$ by

$$
(N C)_{n}=\operatorname{Crs}(\pi[n], C) .
$$

The simplicial operators $(N C)_{m} \rightarrow(N C)_{n}$ are induced by the standard maps $\Delta^{n} \rightarrow \Delta^{m}$. So $N$ is a functor Crs $\rightarrow$ SimpSet. It should be noted that this definition is analogous to that of the well known Dold-Kan functor from chain complexes to simplicial abelian groups.

The crossed complex $\pi[n]$ is a free crossed complex on the cells of $\Delta^{n}$, and the boundaries are determined by the universal example, namely $\delta: \pi[r] \rightarrow \pi[r-1]$, which is itself given by the homotopy addition lemma [115, p.175].

For $n \geqslant 2$ the crossed complex $\pi[n]$ involves non-Abelian groups in dimension 2 and a groupoid in dimension 1 which acts on the groups of the crossed complex. The homotopy addition lemma, which says, intuitively, that the boundary of a simplex is the sum of its faces, therefore needs to be stated with care.

If $\sigma$ is an $r$-simplex with $r \geqslant 4$, then, analogously to the purely additive theory of homology, we have the formula

$$
\delta \sigma=\left(\partial_{0} \sigma\right)^{-a}+\sum_{i=1}^{r}(-)^{i} \partial_{i} \sigma,
$$

where $a=\partial_{2} \partial_{3} \ldots \partial_{r} \sigma$. Here the action of $-a$ transports the base point of $\partial_{0} \sigma$ to the common basepoint 0 of the other faces so as to make addition possible. For a 3 -simplex $\sigma$, we have the non-Abelian formula

$$
\delta \sigma=\left(\partial_{0} \sigma\right)^{-a}+\partial_{2} \sigma-\partial_{1} \sigma-\partial_{3} \sigma
$$


(compare with equation (13)) and for a 2-simplex $\sigma$, we have the groupoid formula

$$
\delta \sigma=\left(\partial_{2} \sigma\right)\left(\partial_{0} \sigma\right)\left(\partial_{1} \sigma\right)^{-1} .
$$

One easily verifies that

$$
\delta \delta \sigma=0
$$

for an $r$-simplex $\sigma$ with $r \geqslant 5$ and that

$$
\delta^{0} \delta \sigma=\delta^{1} \delta \sigma
$$

for a 2-simplex $\sigma$. A direct proof that $\delta \delta \sigma=0$ when $\sigma$ is a 4 -simplex is not so easy, because one has to apply the crossed module rules carefully [115, p.176]. The easiest proof of which I know uses the primary identity property and its characterisation in [43, Prop. 16, p.177]. Cubical versions of the homotopy addition lemma are also well known (see, for example, [35]). It is interesting that a proof of the homotopy addition lemma came quite late in the development of the subject [85]. The proof in [115] assumes all the main results of singular homology together with basic material on relative homotopy groups, and is an inductive proof combined with a proof of the Relative and Absolute Hurewicz Theorems. By contrast, the proof of the cubical homotopy addition lemma and Relative Hurewicz Theorem in $[35,36]$ takes about 60 pages, starting from very little, and is but one application of the Generalised Van Kampen Theorem which yields many more results on the way, including of course results on crossed modules unobtainable from theories dealing only with abelian objects.

The simplicial set $N C$ has an additional structure of thin elements, defined as follows. Let $f: \pi[n] \rightarrow C$ be an element of $(N C)_{n}$. Let $c_{n} \in \pi[n]_{n}$ represent the top dimensional free generator of $\pi[n]$. We say $f$ is thin if $f c_{n}=0$.

Theorem 7.13 The thin elements of NC satisfy the following axioms of K. Dakin [61]:

T1) Any degenerate element of $N C$ is thin.

T2) Any horn in NC has a unique thin filler.

T3) If all faces except possibly one of a thin element are thin, then so also is the remaining face.

The proof of T1) is easy. A proof of T2) can be given using the GVKT (Theorem 7.6) for the homotopy crossed complexes of the skeleta of a $C W$-complex, while a proof of T3) is easy from the homotopy addition lemma.

We say that $N C$ is a simplicial $T$-complex. There is a category SimpT of simplicial $T$-complexes in which the morphisms are simplicial maps which map thin elements to thin elements.

Theorem 7.14 [1] The nerve functor gives an equivalence between the category of crossed complexes and the category of simplicial T-complexes. 
This theorem is quite hard to prove. A version in a general category is given in [98]. The theorem is of course a non abelian analogue of the Dold-Kan theorem which gives an equivalence between chain complexes and simplicial abelian groups. There is a related result giving an equivalence between a category of so-called ' $\omega$-categories' (these are the same as the $\infty$-categories of [38]) and simplicial sets with thin elements satisfying weaker conditions than those given earlier $[109,110]$. Details are not yet available.

Actually there are now a number of categories equivalent to crossed complexes, as shown in the following diagram

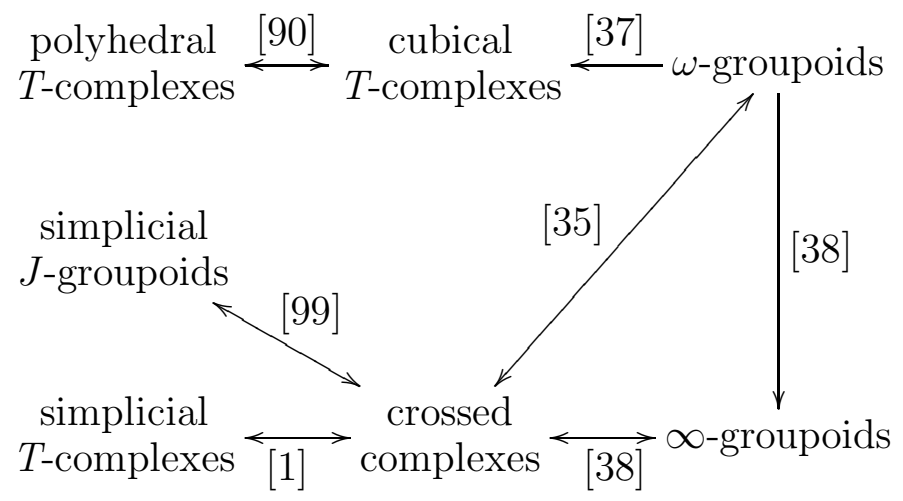

in which each arrow denotes an explicit functor which is an equivalence of categories. The symbols in square brackets give references for the proofs. This splitting of the notion of higher dimensional groups into many equivalent formulations was one of the surprises of the theory and also one of its very useful characteristics. You can carry out a piece of theory or computation in whichever category is convenient for the purposes at hand. The fact that the equivalences are non trivial means that if a result requires a number of these different settings for its proof then you are inputting significant information (namely, the proofs of the equivalences) and the theorem itself is more likely to be significant. From a geometric viewpoint the splitting of higher dimensional group theory into different formulations is not so surprising: it reflects the variety of families of convex polyhedra in dimensions greater than 1 .

The comparative value of crossed complexes is:

i) the free crossed complexes are closely related to, though simpler than, $C W$-complexes in their definition and construction,

ii) they require a smaller amount of data than $\infty$-groupoids, or the other categories, and so are a suitable context for calculations,

iii) there is an explicit description of a useful monoidal closed structure [40], which allows explicit equivalences of the Eilenberg-Zilber type [112],

iv) it is easier to describe the relation with simplicial sets [1, 42], and so the classifying space and homotopy coherence properties $[112,29,30]$, than in the other cases, 
v) relations with the classical homological algebra of chain complexes with operators have been formulated [41].

The comparative value of $\omega$-groupoids is:

i) the cubical structure allows easy formulations of multiple compositions, and so an 'algebraic inverse to subdivision' which is essential for the proofs of the Generalised Van Kampen Theorems [36],

ii) it is easy to formulate the monoidal closed structure in this context,

iii) there is a well worked out relation with cubical sets [36],

iv) there is a renewed interest in cubical methods in areas such as concurrency, and so the introduction of the connections has proved intriguing.

The comparative value of $\infty$-groupoids is as follows:

i) they are a specialisation of the $\infty$-categories [38], which are becoming increasingly used (though often under the name $\omega$-categories, and so it is best to call these globular $\infty$ - or $\omega$-categories to make the meaning clear),

ii) they require a smaller amount of data than the (cubical) $\omega$-categories

iii) they are appropriate for relating with certain weaker structures, the bi- and tri-categor-ies, and various forms of weak $\infty$-categories and groupoids.

Using $\infty$-groupoids in preference to crossed complexes has in some cases disadvantages analogous to those of using equivalence relations, more precisely, congruences, rather than normal subgroups in order to describe quotients of groups. Another difficulty in that there is currently no theory of free $\infty$-groupoids, and the description of tensor products is the so-called Gray tensor product, which does not have convenient formulae. On the other hand, the description of quotients of monoids or categories requires congruences, and relations with weaker structures such as bi-categories seem not possible with crossed complexes. Thus continued experiment is needed.

The relation with simplicial sets and simplicial groupoids is important because of the wide use of simplicial methods in mathematics.

The relation of the 'polyhedral multiple groupoids' of [90] with the 'polyhedral multiple categories' of [108] has not yet been determined.

The geometric realisation of the nerve $N C$ of a crossed complex $C$ gives the classifying space $B C$ of the crossed complex $C$.

If $C$ is a crossed complex, then $C^{(n)}$ denotes the subcrossed complex which coincides with $C$ in dimensions $\leqslant n$ and in higher dimensions is trivial. So we obtain a filtered crossed complex and a corresponding filtered space $\mathcal{B C}=(B C)_{*}$. 
Theorem 7.15 There is natural equivalence of functors $\pi \circ \mathcal{B} \simeq 1$.

The proof goes as follows. The nerve $N C$ of a crossed complex $C$ is a Kan complex, and so the crossed complex $\pi \circ \mathcal{B C}$ may be directly calculated from the corresponding filtration of $N C$. It is then easy to identify the elements of the appropriate relative homotopy groups as represented by morphisms $\pi[n] \rightarrow C$ which give rise to elements of $\mathrm{C}$ by evaluation on the top dimensional cell of $\Delta^{n}$.

Let $X_{*}$ be a filtered space, then we may define $R^{\Delta}\left(X_{*}\right)$ to be the simplicial set of filtered maps $\Delta_{*}^{n} \rightarrow X_{*}, n \geqslant 0$, where $\Delta_{*}^{n}$ denotes the standard $n$-simplex with its skeletal filtration. The quotient of $R^{\Delta}\left(X_{*}\right)$ by the relation of filter homotopy rel vertices is written $\rho^{\Delta}\left(X_{*}\right)$. A basic fact with a tricky proof is that the quotient map $R^{\Delta}\left(X_{*}\right) \rightarrow \rho^{\Delta}\left(X_{*}\right)$ is a Kan fibration (this is proved in [1] modelling the proof in the cubical case given in [36]). This result is used in [1] to prove that $\rho^{\Delta}\left(X_{*}\right)$ has the structure of simplicial $T$-complex whose associated crossed complex is exactly $\pi\left(X_{*}\right)$, so that the underlying simplicial set of $\rho^{\Delta}\left(X_{*}\right)$ is just $N \pi\left(X_{*}\right)$. If $X=X_{\infty}$, then there is an inclusion $i: R^{\Delta}\left(X_{*}\right) \rightarrow S(X)$ where $S(X)$ is the usual singular complex of $X$. There are useful conditions for $i$ to be a homotopy equivalence; for example this is true if $X_{*}$ is the skeletal filtration of a $C W$-complex.

The exact sequence (at some base point) of the fibration $R^{\Delta}\left(X_{*}\right) \rightarrow \rho^{\Delta}\left(X_{*}\right)$ is of interest. In the case $X_{*}$ is the skeletal filtration of a $C W$-complex, it coincides with Whitehead's famous exact sequence [119], as is proved in the cubical case in [36].

\subsection{Homotopies of morphisms of crossed complexes}

This is one of the points where the theory of crossed complexes gets technical, namely in dealing with homotopies. The reason for this is that the natural geometric model for a crossed complex is the $n$-cell $E^{n}$ with the cell structure given by:

$$
E^{n}= \begin{cases}e^{0} & \text { if } n=0, \\ e_{+}^{0} \cup e_{-}^{0} \cup e^{1} & \text { if } n=1, \\ e^{0} \cup e^{n-1} \cup e^{n} & \text { otherwise }\end{cases}
$$

However, the cell structure on a product $E^{n} \times E^{m}$, and in particular on $E^{n} \times E^{1}$ which is the relevant case for homotopies, is more complicated, since $E^{n} \times E^{m}$ has 9 cells for $m, n \geqslant 1$. It is this cell structure on $E^{n} \times E^{m}$ which determines in essence a tensor product $C \otimes D$ of crossed complexes [40], such that

$$
\pi\left(E^{n} \times E^{m}\right) \cong \pi\left(E^{n}\right) \otimes \pi\left(E^{m}\right)
$$

but we have no time to deal with that here.

We follow the conventions for homotopies in [40]. Thus a homotopy $f^{0} \simeq f$ of morphisms $f^{0}, f: C \rightarrow D$ of crossed complexes is a pair $(h, f)$ where $h$ is a family of functions $h_{n}: C_{n} \rightarrow D_{n+1}$ 
with the following properties, in which $\beta c$ for $c \in C$ is $c$, if $c \in C_{0}$, is $\delta^{1} c$, if $c \in C_{1}$, and is $x$ if $c \in C_{n}(x), n \geqslant 2$. So we require [40, (3.1)]:

$$
\begin{aligned}
\beta h_{n}(c) & =\beta f(c) & & \text { for all } c \in C ; \\
h_{1}\left(c c^{\prime}\right) & =h_{1}(c)^{f c^{\prime}} h_{1}\left(c^{\prime}\right) & & \text { if } c, c^{\prime} \in C_{1} \text { and } c c^{\prime} \text { is defined; } \\
h_{2}\left(c c^{\prime}\right) & =h_{2}(c)+h_{2}\left(c^{\prime}\right) & & \text { if } c, c^{\prime} \in C_{2} \text { and } c c^{\prime} \text { is defined; } \\
h_{n}\left(c+c^{\prime}\right) & =h_{n}(c)+h_{n}\left(c^{\prime}\right) & & \text { if } c, c^{\prime} \in C_{n}, n \geqslant 3 \text { and } c+c^{\prime} \text { is defined; } \\
h_{n}\left(c^{c_{1}}\right) & =\left(h_{n} c\right)^{f c_{1}} & & \text { if } c \in C_{n}, n \geqslant 2, c_{1} \in C_{1}, \text { and } c^{c_{1}} \text { is defined. }
\end{aligned}
$$

Then $f^{0}, f$ are related by $[40,(3.14)]$

$$
f^{0}(c)= \begin{cases}\delta^{0} h_{0} c & \text { if } c \in C_{0}, \\ \left(h_{0} \delta^{0} c\right)(f c)\left(\delta_{2} h_{1} c\right)\left(h_{0} \delta^{1} c\right)^{-1} & \text { if } c \in C_{1}, \\ \left\{(f c)\left(h_{1} \delta_{2} c\right)\left(\delta_{3} h_{2} c\right)\right\}_{0}^{\left(h_{0} \beta c\right)^{-1}} & \text { if } c \in C_{2}, \\ \left\{f c+h_{n-1} \delta_{n} c+\delta_{n+1} h_{n} c\right\}^{\left(h_{0} \beta c\right)^{-1}} & \text { if } c \in C_{n}, n \geqslant 3 .\end{cases}
$$

The set of homotopy classes of crossed complex morphisms $C \rightarrow D$ is written $[C, D]$. If $X, Y$ are spaces, then the set of homotopy classes of maps $X \rightarrow Y$ is also written $[X, Y]$. The main homotopy classification theorem for crossed complexes is:

Theorem 7.16 (Brown and Higgins [42]) If $X_{*}$ is the skeletal filtration of a $C W$-complex, and $C$ is a crossed complex, then there is a natural bijection of sets

$$
\phi:[X, B C] \cong\left[\pi X_{*}, C\right] .
$$

Note that this bijection gives a translation from topology on the left to algebra on the right, since the crossed complex $\pi X_{*}$ has a purely algebraic description which makes it analogous to the chain complex of cellular chains of the CW-complex $X$. The theorem gives a kind of 'homotopy adjointness' of $\pi$ and $B$. It includes many classical classification theorems, including the case of maps into an Eilenberg-Mac Lane space $K(G, n), n \geqslant 1$ and also the case of local coefficients, by taking suitable choice of $C$. For more details, see [42].

The proof of the theorem involves setting up a monoidal closed structure on the category Crs, with an internal hom $C R S(-,-)$ and tensor product $-\otimes-$ and natural equivalence

$$
\operatorname{Crs}(A \otimes B, C) \cong \operatorname{Crs}(A, C R S(B, C))
$$

for any crossed complexes $A, B, C$. There is in this category a model $\mathcal{I}$ of the unit interval, and this allows us to realise homotopies as morphisms $B \otimes \mathcal{I} \rightarrow C$. In this way we can establish much of the basic machinery of homotopy theory (cylinder objects, path objects, fibrations, cofibrations, and so on) in the category of crossed complexes. This extra structure is exploited in [41, 42, 28, 29]. The last paper deals explicitly with notions of homotopy coherence for crossed complexes. 
Also necessary is the Eilenberg-Zilber theorem for crossed complexes, describing the crossed complex $\pi(|K \times L|)$ of the geometric realisation of a product of simplicial sets as homotopy equivalent to $\pi(|K|) \otimes \pi(|L|)$. This result is proved in detail in [112] and given applications in [42, 112, 29, 30]. Many specific properties of the Eilenberg-Zilber equivalence are used in the last paper to describe and prove the homotopy coherence properties of the adjoint functors $N:$ Crs $\rightarrow \operatorname{Simp}, \pi:$ Simp $\rightarrow$ Crs. Such results would seem to be more difficult to establish and apply for (globular) $\infty$-groupoids than for crossed complexes.

Methods of contracting homotopies on universal covers of free crossed complexes are used in [49] to construct inductively free crossed resolutions of groups from a partial free crossed resolution, and in particular from a presentation of a group. This gives a geometric formula for a presentation of the module of identities among relations for a group presentation.

\subsection{Relation with chain complexes with operators}

There is a functor $\mathcal{D}$ from crossed complexes to chain complexes of modules, described for free reduced crossed complexes by Whitehead in [118], and for general crossed complexes in [41]. For simplicity we first describe this in the reduced case.

Given a reduced crossed complex as in the top line of the following diagram, we will obtain the chain complex of modules in the bottom line and the diagram of functions between them:

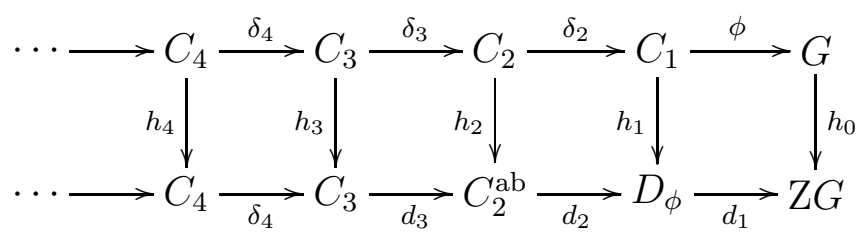

Here $G=\pi_{1} C=$ Coker $\delta_{2}$ and $\phi: C_{1} \rightarrow G$ is the quotient map. For $i \geqslant 3, h_{i}$ is the identity morphism, $h_{2}$ is abelianisation, and $d_{3}=h_{2} \delta_{3}$. The function $h_{0}$ is given by $g \mapsto g-1$. Then $h_{0}$ is a derivation: $h_{0}(x y)=\left(h_{0} x\right) y+h_{0} y$, for all $x, y \in G$. That leaves the definition of $D_{\phi}, h_{1}, d_{2}, d_{1}$.

The function $h_{1}: C_{1} \rightarrow D_{\phi}$ is defined to be the universal $\phi$-derivation, by which is meant

(i) $D_{\phi}$ is a $G$-module and $h_{1}(a b)=\left(h_{1} a\right)^{\phi b}+h_{1}(b)$, for all $a, b \in C_{1}$, and

(ii) $h_{1}$ is universal for property (i), so that if $k: C_{1} \rightarrow M$ is a $\phi$-derivation to a $G$-module $M$, then there is a unique $G$-module morphism $k^{\prime}: D_{\phi} \rightarrow M$ such that $k^{\prime} h_{1}=k$.

This definition is due to Crowell [59]. He calls $M_{\phi}$ the derived module of the morphism $\phi$, and constructs it as the quotient of the free $G$-module on elements $[a], a \in C_{1}$, by the relations $[a b]-$ $[a]^{\phi b}-[b]$ for all $a, b \in C_{1}$. 
If $C_{1}$ is a free group on a set $X$, then a $\phi$-derivation $k: C_{1} \rightarrow M$ is entirely determined by its values on the basis $X$ : the easiest way to see this is to regard a $\phi$-derivation as a section of the projection $p_{1}: C_{1} \ltimes M \rightarrow C_{1}$ where $C_{1}$ acts on $M$ via $\phi$.

It is easily verified that $h_{0}: G \rightarrow I G, g \mapsto g-1$, is the universal $1_{G}$-derivation. In fact we may also construct the derived module as $I C_{1} \otimes_{C_{1}} \mathrm{Z} G, a \mapsto\left(a-1_{C_{1}}\right) \otimes 1_{G}$.

It is useful that if we regard $D$ as a functor Groups ${ }^{2} \rightarrow$ Mod from the category of morphisms of groups to the category of modules, then $D$ has a right adjoint

$$
(H, M) \mapsto\left(H \ltimes M \stackrel{p_{1}}{\longrightarrow} H\right)
$$

where $p_{1}$ is the first projection of the semidirect product. It follows that $D$ preserves colimits. It is an easy deduction from this that if $C_{1}$ is a free group on a set $X$, then $D_{\phi}$ is isomorphic to the free $G$-module on the set $X$, with $h_{0}$ given by $x \mapsto x, x \in X$.

The following result is useful.

Proposition 7.17 [41] The morphisms of $G$-modules $d_{2}, d_{1}$ in diagram (44) may be defined to give a commutative diagram. Further, if the sequence of groups

$$
C_{3} \stackrel{\delta_{3}}{\longrightarrow} C_{2} \stackrel{\delta_{2}}{\longrightarrow} C_{1} \stackrel{\phi}{\longrightarrow} G \rightarrow 1
$$

is exact, so also is the sequence

$$
C_{3} \stackrel{d_{3}}{\longrightarrow} C_{2}^{\mathrm{ab}} \stackrel{d_{2}}{\longrightarrow} D_{\phi} \stackrel{d_{1}}{\longrightarrow} I G \rightarrow 0,
$$

and $\operatorname{Im} d_{2} \cong(\operatorname{ker} \phi)^{\mathrm{ab}}$.

The proof is given in the more general non reduced case in [41].

Parts of the exact sequence (46) occurs in many instances in the homological algebra of groups. In the case that $C_{2} \rightarrow C_{1}$ is the free crossed module over a free group derived from a presentation $(X ; R)$ of the group $G$, then the morphism of $G$-modules $d_{2}$ agrees with the Fox derivative in the free differential calculus

$$
(\partial r / \partial x):(\mathrm{Z} G)^{R} \rightarrow(\mathrm{Z} G)^{X} .
$$

The construction of this derivative from the free crossed module over a free group in the above way occurs in Whitehead's paper [118, Lemma 8] which in fact antedates Fox's paper on this subject [72]. See also [43] for an exposition.

It is important that Whitehead's work in [118] also dealt with the relations between homotopies in the categories of crossed complexes and of chain complexes with operators, and this aspect is studied also in [41]. In particular, Whitehead obtains a homotopy classification

$$
[X, Y] \cong[C(\widetilde{X}), C(\tilde{Y})]
$$


for reduced $C W$-complexes $X, Y$ such that $X$ is $n$-dimensional and $\pi_{i}(Y)=0,1 \leqslant i<n$, and where $C(\widetilde{X})$ denotes the chain complex of cellular chains of the universal cover of $X$, with its operation of the fundamental group of $X$. However the result (47) is not stated explicitly in [118]. It is interesting that this result includes homotopy classification results of Olum and others which were published later and were formulated simplicially rather than cellularly. An exposition of this method with applications is given in [68].

Further applications of the relations between crossed complexes and chain complexes with operators established in [41] are given in [9, 10].

\subsection{Some computations of Postnikov invariants of crossed modules}

The exposition here is taken from [52].

Recall $[87,14]$ that if $G$ is a group and $A$ is a $G$-module, then elements of $H^{3}(G, A)$ may be represented by equivalence classes of crossed sequences

$$
0 \rightarrow A \rightarrow M \stackrel{\mu}{\longrightarrow} P \rightarrow G \rightarrow 1
$$

namely exact sequences as above such that $(\mu: M \rightarrow P)$ is a crossed module. The equivalence relation between such crossed sequences is generated by the basic equivalences, namely the existence of a commutative diagram of morphisms of groups as follows

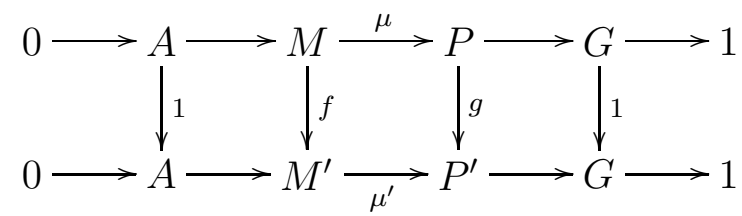

such that $(f, g)$ is a morphism of crossed modules. Such a diagram is called a morphism of crossed sequences.

The zero cohomology class is represented by the crossed sequence

$$
0 \rightarrow A \stackrel{1}{\longrightarrow} A \stackrel{0}{\longrightarrow} G \stackrel{1}{\longrightarrow} G \rightarrow 1
$$

which we sometimes abbreviate to

$$
A \stackrel{0}{\longrightarrow} G
$$

In a similar spirit, we say that a crossed module $(\mu: M \rightarrow P)$ represents a cohomology class, namely an element of $H^{3}$ (Coker $\mu$, Ker $\mu$ ). Although this equivalence between classes of crossed sequences and 3-dimensional cohomology classes has been known for a long time, it is not so easy to find calculations of examples, and so we give some in this section. 
Example 7.18 Let $C_{n^{2}}$ denote the cyclic group of order $n^{2}$, written multiplicatively, with generator $u$. Let $\gamma_{n}: C_{n^{2}} \rightarrow C_{n^{2}}$ be given by $u \mapsto u^{n}$. This defines a crossed module, with trivial operations. This crossed module represents the trivial cohomology class in $H^{3}\left(C_{n}, C_{n}\right)$, in view of the morphisms of crossed sequences

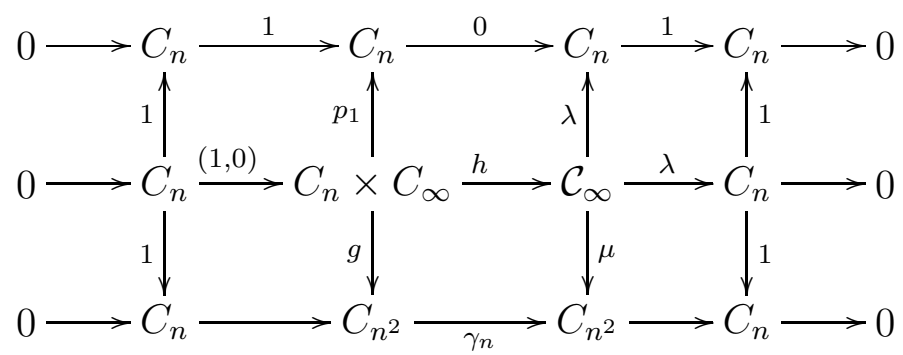

where, if $t$ denotes a generator of any cyclic group, then $g(t, 1)=t^{n}, g(1, t)=t, h(t, 1)=1, h(1, t)=$ $t^{n}$ and $\lambda, \mu$ are surjections.

Example 7.19 We show that the dihedral crossed module $\mathcal{D}_{n}$ of Example 5.7 represents the trivial cohomology class. This is clear for $n$ odd, since then $\delta$ is an isomorphism. For $n$ even, we simply construct a morphism of crossed sequences as in the following diagram

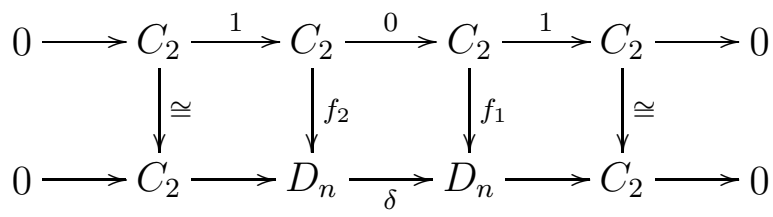

where if $t$ denotes the non trivial element of $C_{2}$ then $f_{1}(t)=x, f_{2}(t)=u^{n / 2}$. Just for interest, we leave it to the reader to prove that there is no morphism in the other direction between these crossed sequences.

A crossed module $\mathcal{M}=(\mu: M \rightarrow P)$ determines a cohomology class

$$
k_{\mathcal{M}} \in H^{3}(\text { Coker } \mu \text {, Ker } \mu) \text {. }
$$

The addition of such cohomology classes is determined by a sum of crossed sequences, of the Baer type. An exposition of this is given, for example, by Danas in [62].

If $X$ is a connected, pointed $C W$-complex with 1-skeleton $X^{1}$, then the class

$$
k_{X}^{3} \in H^{3}\left(\pi_{1} X, \pi_{2} X\right)
$$

of the crossed module $\Pi_{2}\left(X, X^{1}\right)$ is called the first Postnikov invariant of $X$. This class is also represented by $\Pi_{2}(X, A)$ for any connected subcomplex $A$ of $X$ such that $(X, A)$ is 1-connected and $\pi_{2}(A)=0$. It may be quite difficult to determine this Postnikov invariant from a presentation of 
this last crossed module, and even the meaning of the word "determine" in this case is not so clear. There are practical advantages in working directly with the crossed module, since it is an algebraic object, and so it, or families of such objects, may be manipulated in many convenient and useful ways. Thus the advantages of crossed modules over the corresponding 3-cocycles are analogous to some of the advantages of homology groups over Betti numbers and torsion coefficients.

However, in work with crossed modules, and in applications to homotopy theory, information on the corresponding cohomology classes, such as their non-triviality, or their order, is also of interest. The aim of this section is to give background to such a determination, and to give two example of finite crossed modules representing non-trivial elements of the corresponding cohomology groups.

It has been proved by Ellis [70] that if $G, A$ are finite, where $A$ is a $G$-module, then every element of $H^{3}(G, A)$ can be represented by a finite crossed module.

The natural context in which to show how a crossed sequence gives rise to a 3-cocycle is not the traditional chain complexes with operators but that of crossed complexes, as shown in [87]. We explain how this works here. For more information on the relations between crossed complexes and the traditional chain complexes with operators than given here, see again [41], and for the relation with local cohomology, see [42].

A free crossed resolution of the group $G$ is a free aspherical crossed complex $F_{*}$ together with an epimorphism $\phi: F_{1} \rightarrow G$ with kernel $\delta_{2}\left(F_{2}\right)$. Here aspherical means that $\operatorname{Im} \delta_{n+1}=\operatorname{ker} \delta_{n}$ for $n \geqslant 2$.

Example 7.20 The cyclic group $C_{n}$ of order $n$ is written multiplicatively, with generator $t$. We give for it a free crossed resolution $F_{*}$ as follows. Set $F_{1}=C_{\infty}$, with generator $w$, and for $r \geqslant 2$, set $F_{r}=\left(C_{\infty}\right)^{n}$. Here for $r \geqslant 2, F_{r}$ is regarded as the free $C_{n}$-module on one generator $w_{0}$, and we set $w_{i}=\left(w_{0}\right)^{t^{i}}$. The morphism $\phi: C_{\infty} \rightarrow C_{n}$ sends $w$ to $t$, and the operation of $F_{1}$ on $F_{r}$ for $r \geqslant 2$ is via $\phi$. The boundaries are given by

(i) $\delta_{2}\left(w_{i}\right)=w^{n}$,

(ii) for $r$ odd, $\delta_{r}\left(w_{i}\right)=w_{i} w_{i+1}^{-1}$,

(iii) for $r$ even and greater than $2, \delta_{r}\left(w_{i}\right)=w_{0} w_{1} \ldots w_{n-1}$.

Previous calculations show that $\delta_{2}$ is the free crossed $C_{\infty}$-module on the element $w^{n} \in C_{\infty}$. Thus $F_{*}$ is a free crossed complex. It is easily checked to be aspherical, and so is, with $\phi$, a crossed resolution of $C_{n}$.

Let $A$ be a $G$-module. Let $C(G, A, 3)$ denote the crossed complex $C$ which is $G$ in dimension 1 , $A$ in dimension 3, with the given action of $G$ on $A$, and which is 0 elsewhere, as in the following diagram

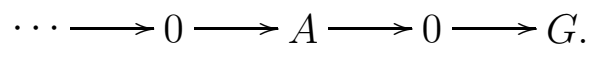


Let $\left(F_{*}, \phi\right)$ be a free crossed resolution of $G$. It follows from the discussions in [42, 41] that a 3cocycle of $G$ with coefficients in $A$ can be represented as a morphism of crossed complexes $f: F_{*} \rightarrow$ $C(G, A, 3)$ over $\phi$. This cocycle is a coboundary if there is an operator morphism $l: F_{2} \rightarrow A$ over $\phi: F_{1} \rightarrow G$ such that $l \delta_{3}=f_{3}$.

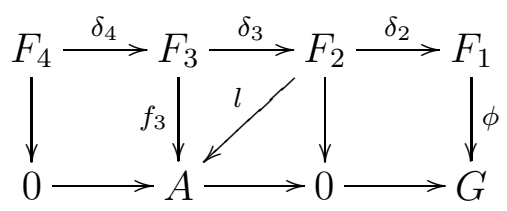

To construct a 3-cocycle on $F_{*}$ from the crossed sequence (48), first construct a morphism of crossed complexes as in the diagram

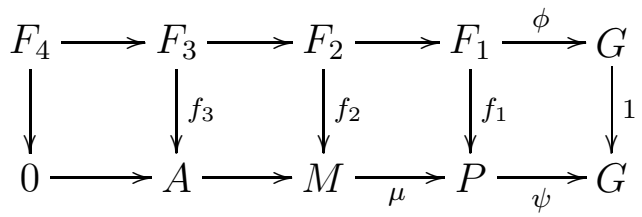

using the freeness of $F_{*}$ and the exactness of the bottom row. Then compose this with the morphism of crossed sequences

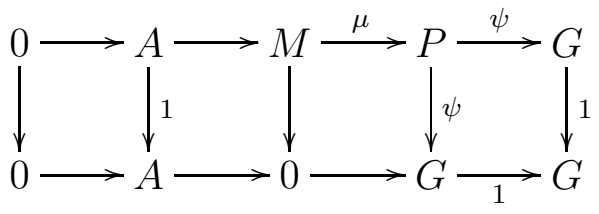

Hence it is reasonable to say that the morphism $f_{3}$ of diagram (49) is a 3 -cocycle corresponding to the crossed sequence.

We now use these methods in an example.

Theorem 7.21 Let $n \geqslant 2$, and let $\iota: C_{n} \rightarrow C_{n^{2}}$ denote the injection sending a generator $t$ of $C_{n}$ to $u^{n}$, where $u$ denotes a generator of $C_{n^{2}}$. Let $A_{n}$ denote the $C_{n}$-module which is the kernel of the induced crossed module $\mathcal{N}=\left(\partial: \iota_{*} C_{n} \rightarrow C_{n^{2}}\right)$. Then $H^{3}\left(C_{n}, A_{n}\right)$ is cyclic of order $n$ and has as generator the class of this induced crossed module.

Proof By Corollary 5.9 the abelian group $\iota_{*} C_{n}$ is the product $V=\left(C_{n}\right)^{n}$. As a $C_{n}$-module it is cyclic, with generator $v$, say. Write $v_{i}=v^{t^{i}}, i=0,1, \ldots, n-1$. Then each $v_{i}$ is a generator of a $C_{n}$ factor of $V$. The kernel $A_{n}$ of $\partial$ is a cyclic $C_{n}$-module on the generator $a=v_{0} v_{1}^{-1}$. Write $a_{i}=a^{t^{i}}=v_{i} v_{i+1}^{-1}$. As an abelian group, $A_{n}$ has generators $a_{0}, a_{1}, \ldots, a_{n-1}$ with relations $a_{i}^{n}=1, a_{0} a_{1} \ldots a_{n-1}=1$. where

We define a morphism $f_{*}$ from $F_{*}$ to the crossed sequence containing $\mathcal{N}$ as in diagram (50), 
(i) $f_{1}$ maps $w$ to $u$,

(ii) $f_{2}$ maps the module generator $w_{0}$ of $F_{2}$ to $v=v_{0}$.

(iii) $f_{3}$ maps the module generator $w_{0}$ of $F_{3}$ to $a_{0}$.

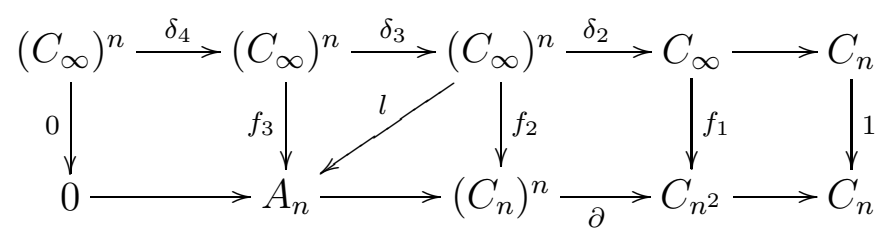

The operator morphisms $f_{r}$ over $f_{1}$ are defined completely by these conditions.

The group of operator morphisms $g:\left(C_{\infty}\right)^{n} \rightarrow A_{n}$ over $f_{1}$ may be identified with $A_{n}$ under $g \mapsto g\left(w_{0}\right)$. Under this identification, the boundaries $\delta_{4}, \delta_{3}$ are transformed respectively to 0 and to $a_{i} \mapsto a_{i}\left(a_{i}^{t}\right)^{-1}$. So the 3 -dimensional cohomology group is the group $A_{n}$ with $a_{i}$ identified with $a_{i+1}, i=0, \ldots, n-1$. This cohomology group is therefore isomorphic to $C_{n}$, and a generator is the class of the above cocycle $f_{3}$.

We now use $\Gamma$ to denote the topological cone functor.

Corollary 7.22 The mapping cone $X=B C_{n^{2}} \cup_{B \iota} \Gamma B C_{n}$ satisfies $\pi_{1} X=C_{n}$, and $\pi_{2} X$ is the $C_{n^{-}}$ module $A_{n}$ of Theorem 7.21. The first Postnikov invariant of $X$ is a generator of the cohomology group $H^{3}\left(\pi_{1} X, \pi_{2} X\right)$, which is a cyclic group of order $n$.

This computation of a cohomology class is generalised in [53] to the case of $B Q \cup \Gamma B P$ where $P$ is a normal subgroup of $Q$ such that $Q / P$ is cyclic of order $n$. We emphasise again that this shows that the 2-dimensional Van Kampen theorem can lead to a complete determination of a non trivial integral homotopy 2-type, and this suggests that the theory has some of the characteristics of a '2-dimensional group theory' sought by the workers in topology near the beginning of this century.

The following is another example of a determination of a non-trivial cohomology class by a crossed module. The method of proof is similar to that of Theorem 7.21, and is left to the reader.

Example 7.23 Let $n$ be even. Let $C_{n}^{\prime}$ denote the $C_{n}$-module which is $C_{n}$ as an abelian group but in which the generator $t$ of the group $C_{n}$ acts on the generator $t^{\prime}$ of $C_{n}^{\prime}$ by sending it to its inverse. Then $H^{3}\left(C_{n}, C_{n}^{\prime}\right) \cong C_{2}$ and a generator of this group is represented by the crossed module $\left(\nu_{n}: C_{n} \times C_{n} \rightarrow C_{n^{2}}\right)$, with generators $t_{0}, t_{1}, u$ say, and where $\nu_{n} t_{0}=\nu_{n} t_{1}=u^{n}$. Here $u \in C_{n^{2}}$ operates by switching $t_{0}, t_{1}$. It is not clear if this crossed module can be an induced crossed module for $n>2$. However, $n=2$ gives the case $n=2$ of Theorem 7.21. 
Remark 7.24 The crossed module $\left(\nu_{2}: C_{2} \times C_{2} \rightarrow C_{4}\right)$ also appears as an example in [85, pp.332333]. The proof given there that its corresponding cohomology class is non-trivial is obtained by relating this class to the obstruction to a certain kind of extension.

From the above we see an advantage in having a small free crossed resolution of a group. This method is used to give computations in non abelian extension theory in [48]. However the methods of constructing such resolutions are at present rather limited, and are the subject of current research $[49,77]$. The problem seems more difficult than the widely researched subject of the computation of resolutions in the usual sense of the cohomology of groups. 


\section{Further work}

The paper [24] lists some 35 problems or problem areas in non Abelian homological and homotopical algebra related to the work described here.

A general problem seems to be that of linking these methods with other techniques and problems in algebraic topology. There is a considerable body of work by Graham Ellis and his collaborators on applying in group theory techniques related to the non abelian tensor product. This gives a lot of new results on $p$-groups and their homology, and on combinatorial homotopy. This is related to the long term programme by Baues on the classification problem in topology.

The category of crossed complexes has many satisfactory properties. Its monoidal closed structure [38] has been exploited in for example $[27,42,9,7,29,10]$. This work is probably the beginning of work on crossed differential algebras to be exploited in the homotopy theory of non simply connected spaces. That is, there seems a lot more to be done and to be achievable in moving to the quadratic homotopical information. At a low dimensional level this has been done in [6] using quadratic complexes, and from the viewpoint of crossed squares in [69]. Are crossed differential algebras the 'right' quadratic model for all dimensions?

The paper [21] gives a general overview of the methods of crossed squares and crossed $n$-cubes of groups. A striking application of the Van Kampen Theorem for crossed squares and which cannot be obtained from using crossed complexes is the determination of $\pi_{3} S K(G, 1)$, where $S$ denotes suspension, as the kernel of a morphism determined by the commutator map $G \times G \rightarrow G$ [44]. Here the non abelian tensor square is universal for 'biderivations' on $G \times G$. This has led to a lot of work on non abelian tensor squares and tensor products, and so to a number of explicit calculations of $G \otimes G$ and so of the kernel of the commutator map $\kappa: G \otimes G \rightarrow G$.

Note that, analogously to the case of induced crossed modules, a standard group theory construction, in this case the commutator subgroup, is replaced by an in general bigger group, the tensor square, with a universal property, and that the kernel of the morphism from the universal construction to the standard construction contains information on absolute homotopy groups. Further, the route to these applications is through Generalised Van Kampen Theorems, whose methods of proof require higher order groupoids.

It is a consequence of work of Loday [94] that homotopy $n$-types are modelled by $n$-fold groupoids. Grothendieck described this result to me as 'Absolutely beautiful!'. There is a lot more work to be done to develop the consequences. For example, this result is so far of little use in describing the $(n+2)$-type of $S^{n}$.

There is also the problem of exploiting these methods in other areas of algebraic and differential topology, granted the importance of the general notions of deformation and homotopies. The work of Baues in setting up homotopy theory in a cofibration category is important here.

We return to the idea that structures which can be the target of a functor satisfying a Van Kampen type theorem have some special properties which make them suitable for a non abelian 
local-to-global theorem. It would be good if these properties could be exploited in other situations than has been done so far.

Some wider general issues are also given in [23] and in the less technical web page article [25].

Finally I must acknowledge with pleasure the very enjoyable collaborations which will be evident from the publication list and the work of research students at Bangor who have made key contributions to the subject of groupoids, multiple groupoids and related areas, under the supervision and of and through discussion with Tim Porter, Chris Wensley and the writer. Here are their names with dates of completion: Lew Hardy (1974), Tony Seda (1974), A. Razak Salleh (1975), Keith Dakin (1976), Nick Ashley (1978), David Jones (1984), Graham Ellis (1984), Fahmi Korkes (1985), Mohammed Aof (1988), Fahd Al-Agl (1990), John Shrimpton (1990), Osman Mucuk (1993), Andy Tonks (1993), Phil Ehlers (1994), Zaki Arvasi (1995), Ilhan İçen (1996), Murat Alp (1997), Ali Mutlu (1997), Anne Heyworth (1998).

This article is a slightly revised version of about nine hours of lectures given at the Summer School on the Foundations of Algebraic Topology, Grenoble, June 16 - July 4, 1997. I would like to thank Francis Sergeraert for the organisation of this School, and the Université Joseph Fourier, Grenoble, for support. 


\section{References}

[1] Ashley, N.K., Simplicial T-complexes, PhD. Thesis, University of Wales, 1978. Published as Simplicial T-complexes: a non-abelian version of a theorem of Dold-Kan, Diss. Math. 265, 11-58 (1988).

[2] Baez, J. C. and Neuchl, M., 'Higher dimensional algebra I: Braided monoidal 2-categories', Adv. Math., 121 (1996) 196-244.

[3] Baez, J. C. and Dolan, J., 'Higher-dimensional algebra III: $n$-categories and the algebra of Opetopes',Adv. Mathematics, 135, (1998) 145-206.

[4] Baik, Y. G., Harlander, J. and Pride, S.J., 'The geometry of group extensions', J. Group Theory, 1 (1998) 395-416.

[5] Baik, Y.G., and Pride, S.J., 'Generators of the second homotopy module of presentations arising from group constructions', Preprint, Glasgow 1992.

[6] Baues, H.J., Combinatorial homotopy and 4-dimensional complexes, De Gruyter, 1991.

[7] Baues, H.J. and Brown, R., 'On the relative homotopy groups of the product filtration and a formula of Hopf', J. Pure Appl. Algebra 89 (1993) 49-61.

[8] Baues, H.J.,Conduché, D., 'The central series for Peiffer commutators in groups with operators', J.Algebra, 133, 1-34, 1990.

[9] Baues, H.J., Conduché, D., 'The tensor algebra of a non-Abelian group', K-theory, 5 (1992) 531-554

[10] Baues, H. J., Tonks, A., 'On the twisted cobar construction', Math. Proc. Camb. Phil. Soc., 121 (1997) 229-245.

[11] Blakers, A.L., 'Relations between homology and homotopy groups', Annals of Math., 49 (1948) 428461.

[12] Bourbaki, N., Élements de mathématique, Livre II: Algèbre, Ch. I: Structures algébriques, Hermann, Paris, (1964).

[13] Breen, L., 'Théorie de Schreier supérieure', Ann. Sci. Écol. Norm. Sup., 25 (1992) 465-514.

[14] Brown, K.S., Cohomology of groups, Graduate texts in Mathematics 87, Springer-Verlag, New York (1982).

[15] Brown, R., 'Groupoids and Van Kampen's theorem', Proc. London Math. Soc., (3) 17 (1967) 385-340.

[16] Brown, R., Topology: a geometric account of general topology, homotopy types and the fundamental groupoid, Ellis Horwood, Chichester, (1988) (first edition, McGraw Hill, 1967).

[17] Brown, R., 'Fibrations of groupoids', J. Algebra 15 (1970) 103-132.

[18] Brown, R., 'Coproducts of crossed P-modules: applications to second homotopy groups and to the homology of groups', Topology 23, 337-345, 1984.

[19] Brown, R., 'From groups to groupoids: a brief survey', Bull. London Math. Soc., 19 (1987) 113-134.

[20] Brown, R., 'Triadic Van Kampen theorems and Hurewicz theorem', Algebraic Topology, Proc. Int. Conf. March 1988, Edited M.Mahowald and S.Priddy, Cont. Math. 96 (1989) 39-57. 
[21] Brown,R., 'Computing homotopy types using crossed n-cubes of groups', Proc. Adams Memorial Symposium on Algebraic Topology, Manchester 1990, Volume I, ed N.Ray and G. Walker, London Math. Soc. Lecture Note Series 175, Cambridge University Press, 187-210, 1991.

[22] Brown, R., 'Higher order symmetry of graphs', Bull. Irish Math. Soc. 32 (1994) 46-59.

[23] Brown, R., 'Homotopy theory, and change of base for groupoids and multiple groupoids', Applied categorical structures, 4 (1996) 175-193.

[24] Brown, R. 'Some problems in non-Abelian homological and homotopical algebra', Homotopy theory and related topics: Proceedings Kinosaki, 1988, Edited M.Mimura, Springer Lecture Notes in Math. 1418, 105-129, 1990.

[25] Brown, R., 'Higher dimensional group theory', http://www.bangor.ac.uk/ mas010/hdaweb2.htm, 1997.

[26] Brown, R. and Ellis, G.J., 'Hopf formulae for the higher homology of a group', Bull. London Math. Soc., 20 (1988) 124-128.

[27] Brown, R., Gilbert, N.D., 'Algebraic models of 3-types and automorphism structures for crossed modules', Proc. London Math. Soc. (3) 59, 51-73, 1989.

[28] Brown,R. and Golasinski, M., 'A model structure for the homotopy theory of crossed complexes', Cah. Top. Géom. Diff. Cat. 31, 61-82, 1989.

[29] Brown, R., Golasinski, M., Porter, T. and Tonks, A., 'On function spaces of equivariant maps and the equivariant homotopy theory of crossed complexes' Indag. Math. 8 (1997) 157-172.

[30] Brown, R., Golasinski, M., Porter, T. and Tonks, A., 'On function spaces of equivariant maps and the equivariant homotopy theory of crossed complexes II: the case of general topological groups' (submitted).

[31] Brown, R. and Heath, P.R., 'Cogluing homotopy equivalences', Math. Z. 113 (1970) 313-362.

[32] Brown, R. and Heath, P.R., 'Lifting amalgamated sums and other colimits of groups and topological groups', Math. Proc. Camb. Phil. Soc., 102 (1987) 273-280.

[33] Brown, R., Heath, P.R. and Kamps, H., 'Groupoids and the Mayer-Vietoris sequence', J. Pure Appl. Alg. 30 (1983) 109-129.

[34] Brown, R. and Higgins, P.J., 'On the connection between the second relative homotopy groups of some related spaces', Proc. London Math. Soc. , (3) 36 (1978) 193-212.

[35] Brown, R. and Higgins, P.J., 'On the algebra of cubes', J. Pure Appl. Algebra 21 (1981) 233-260.

[36] Brown, R. and Higgins, P.J., 'Colimit theorems for relative homotopy groups', J. Pure Appl. Algebra 22 (1981) 11-41.

[37] Brown, R. and Higgins, P.J., 'The equivalence of $\omega$-groupoids and cubical T-complexes', Cah. Top. Géom. Diff. Cat. 22 (1981) 349-370.

[38] Brown, R. and Higgins, P.J., 'The equivalence of $\infty$-groupoids and crossed complexes', Cah. Top. Géom. Diff. Cat., 22 (1981) 371-386.

[39] Brown, R. and Higgins, P.J.,'Crossed complexes and non-abelian extensions', In Proc. International Conference on Category Theory: Gummersbach, 1981, Lecture Notes in Math. vol. 962 (SpringerVerlag, 1982), 39-50. 
[40] Brown, R. and Higgins, P.J., 'Tensor products and homotopies for $\omega$-groupoids and crossed complexes', J. Pure Appl. Algebra, 47 (1987) 1-33.

[41] Brown, R. and Higgins, P.J., 'Crossed complexes and chain complexes with operators', Math. Proc. Camb. Phil. Soc., 107 (1990) 33-57.

[42] Brown, R. and Higgins, P.J., 'The classifying space of a crossed complex', Math. Proc. Camb. Phil. Soc., 110 (1991), 95-120.

[43] Brown, R. and Huebschmann, J., 'Identities among relations', in Low-dimensional topology, ed. R.Brown and T.L.Thickstun, London Math. Soc. Lect. Notes 46, Cambridge University Press, (1982) 153-202.

[44] Brown, R. and Loday, J.-L., 'Van Kampen Theorems for diagrams of spaces', Topology, 26, (1987) 311-335.

[45] Brown, R. and Loday,J.-L., 'Homotopical excision, and Hurewicz theorems, for $n$-cubes of spaces', Proc. London Math. Soc. (3) 54 (1987) 176-192.

[46] Brown, R. and Mackenzie, K.C.H, 'Determination of a double Lie groupoid by its core diagram', J. Pure Appl. Algebra, 80 (1992) 237-272.

[47] Brown, R. and Mucuk, O., 'Covering groups of non-connected topological groups revisited', Math. Proc. Camb. Phil. Soc. 115 (1994) 97-110.

[48] Brown, R. and Porter, T., 'On the Schreier theory of non-abelian extensions: generalisations and computations', Proceedings Royal Irish Academy, 96 (1996) 213-227.

[49] Brown, R. and Razak Salleh, A., 'Free crossed resolutions of groups and presentations of modules of identities among relations', Bangor Preprint 98.24, 36pp.

[50] Brown, R. and Spencer, C.B., 'Double groupoids and crossed modules', Cah. Top. Géom. Diff., 17 (1976) 343-362.

[51] Brown, R. and Spencer, C.B., ' $\mathcal{G}$-groupoids, crossed modules and the fundamental groupoid of a topological group', Proc. Kon. Ned. Akad. v. Wet., 79 (1976) 296-302.

[52] Brown, R. and Wensley, C.D., 'On finite induced crossed modules and the homotopy 2-type of mapping cones', Theory and applications of categories, 1 (1995) 51-74.

[53] Brown, R. and Wensley, C.D., 'Computing crossed modules induced by an inclusion of a normal subgroup, with applications to homotopy theory', Theory and applications of categories, 2 (1996) 3-16.

[54] Brown, R. and Wensley, C.D., 'Induced crossed modules and computational group theory', UWB Preprint 95.05.

[55] Carrasco, P., Cegarra, A.M., 'Group-theoretic algebraic models for homotopy types', J. Pure Appl. Algebra, 75 (1991) '95-235.

[56] Cathelineau, J.-P., 'Birapport et groupoïdes', Enseign. Math., II. Ser. 41, No.3-4, (1995) 257-280.

[57] Čech, E. 'Höherdimensionale homotopiegruppen', Verhandlungen des Internationalen MathematikerKongresses Zurich, Band 2, (1932) 203.

[58] Connes, A., Non commutative geometry, Academic Press (1994). 
[59] Crowell, R.H., The derived module of a homomorphism, Adv. Math. 6 (1971) 210-238.

[60] Crowell, R., and Fox, R.H., Introduction to knot theory, Boston, Ginn (1963)

[61] Dakin, K., Kan complexes and multiple groupoid structures, Ph.D. thesis, University of Wales (1977).

[62] Danas, G., Group extensions and cohomology in cartesian closed categories, PhD Thesis, City University of New York, (1991).

[63] J.Dieudonné, A history of algebraic and differential topology 1900-1960, Birkhauser, Boston-Basel, 1989.

[64] Ehlers., P.J., Algebraic homotopy of simplicially enriched groupoids, Ph. D. Thesis, University of Wales, 1993.

[65] Ehresmann, C., Catégories et structures, Dunod, Paris, (1964).

[66] Ehresmann, C., Oeuvres complètes et commentées, ed Andrée C. Ehresmann, Suppl. Cah. Top. Géom. Diff. Cat. Amiens, (1980-84).

[67] Ellis, G.J., 'The non-Abelian tensor product of finite groups is finite', J. Algebra 111 (1987) 203-205.

[68] Ellis, G.J., 'Homotopy classification the Whitehead way', Exposit. Math. 6 (1988) 97-110.

[69] Ellis, G.J., 'Crossed squares and combinatorial homotopy', Math. Z. 214 (1993) 93-110.

[70] Ellis, G.J., 'Spaces with finitely many non trivial homotopy groups all of which are finite', Topology, 36 (1997) 501-504.

[71] Ellis, G.J. and Steiner, R., 'Higher-dimensional crossed modules and the homotopy groups of $(n+1)$ ads', J. Pure Appl. Algebra, 46 (1987) 117-136.

[72] Fox, R.H., 'Free differential calculus I: Derivations in the group ring', 'II: The isomorphism problem', 'III: Subgroups', Ann. Math. 57 (1953) 547-560, 59 (1954) 196-210, 64 (1956) 407-419.

[73] Grothendieck, A., private communication, (1985).

[74] Gruenberg, K.W., 'Resolutions by relations', J. London Math. Soc., 35 (1960) 481-494.

[75] Hasse, M., 'Einige Bemerkungen über Graphen, Kategorien und Gruppoide', Math. Nachr., 22 (1960) 255-270.

[76] Heath, P.R. and Kamps, H.K., 'On exact orbit sequences', Ill. J. Math. 26 (1982) 187-191.

[77] Heyworth, Anne, Applications of Rewriting Systems and Gröbner Bases to Computing Kan Extensions and Identities Among Relations, University of Wales, Bangor, PhD. Thesis, (1998).

http://xxx.soton.ac.uk/abs/math.CT/9812097

[78] Higgins, P.J., 'Presentations of groupoids, with applications to groups', Proc. Camb. Phil. Soc., 60 (1964) 7-20.

[79] Higgins, P.J., 'On Grushko's theorem', J. Algebra 4 (1966) 365-372.

[80] Higgins, P.J., Categories and groupoids Van Nostrand, (1971).

[81] Higgins, P.J. and Taylor, J., 'The fundamental groupoid and homotopy crossed complex of an orbit space', Category Theory Proceedings, Gummersbach, 1981, Lecture Notes in Math. 962, edited K.H.Kamps et al, Springer, Berlin, pp. 115-122, 1982.

[82] Hilton, P.J. and Wylie, S., Homology theory, Cambridge Univ. Press, 1960. 
[83] Hog-Angeloni, C., Metzler, W. and Sieradski, A.J. (Editors), Two-dimensional homotopy and combinatorial group theory, London Math. Soc. Lecture Note Series 197, Cambridge University Press, Cambridge (1993).

[84] Howie, J., 'Pullback functors and crossed complexes', Cahiers Topologie Géom. Différentielle Catégoriques 20 (1979), 281-295.

[85] Hu, S.T., 'The homotopy addition theorem', Ann. Math. 58 (1953) 108-122.

[86] Hu, S.T., Homotopy theory (Academic Press, 1959).

[87] Huebschmann, J., 'Crossed n-fold extensions and cohomology, Comm. Math. Helv., 55 (1980) 302-314.

[88] Huebschmann, J., Automorphisms of group extensions and differentials in the Lyndon-HochschildSerre spectral sequence, J. Algebra, 72 (1981) 296-334.

[89] Kamps, H., tom Dieck, T. and Puppe, D., Homotopietheorie, Springer Lecture Notes in Mathematics,

[90] Jones, D.W., Polyhedral T-complexes, University of Wales PhD Thesis, 1984; published as A general theory of polyhedral sets and their corresponding T-complexes, Diss. Math. 266, 1988.

[91] Kamps, H.K. and Porter, T., Abstract homotopy theory, World Scientific (1977).

[92] Kelly, G.M., and Street, R., 'Review of the elements of 2-categories', in Springer Lecture Notes in Math. 420 (1974) 75-103.

[93] Kneser, M., Knus, M.-A., Ojanguren, M., Parimala, R., and Sridharan, R., 'Composition of quaternary quadratic forms, Comp. Math., 60 (1986) 133-150.

[94] Loday, J.-L., 'Spaces with finitely many non-trivial homotopy groups', J. Pure Appl. Algebra, 24 (1982) 179-202.

[95] Mac Lane, S. and Moerdijk, I., Sheaves in Geometry and Logic: a first introduction to topos theory, Springer-Verlag (1992).

[96] Mac Lane, S. and Whitehead, J.H.C., 'On the 3-type of a complex', Proc. Nat. Acad. Sci. (1950) 41-48.

[97] Moerdijk, I., and Svensson, J.-A., Algebraic classification of equivariant homotopy 2-types, I, J.Pure Applied Alg., 89 (1993) 187-216.

[98] Nan Tie, G., 'A Dold-Kan theorem for crossed complexes', J. Pure Appl. Algebra, 56 (1989) 177-194.

[99] Nan Tie, G., 'Crossed complexes and simplicial J-groupoids', J. Pure Appl. Algebra, 56 (1989) 195-222.

[100] Poincaré, H. (1895).

[101] Porter, T., ' $n$-types of simplicial groups and crossed n-cubes', Topology 32 (1993) 5-24.

[102] Pride, S.J., 'Identities among relations', in Proc. Workshop on Group Theory from a Geometrical Viewpoint, eds. E.Ghys, A.Haefliger, A. Verjodsky, International Centre of Theoretical Physics, Trieste, 1990, World Scientific (1991) 687-716.

[103] Razak Salleh, A., Union theorems for double groupoids and groupoids; some generalisations and applications, PhD Thesis, University of Wales, 1976. 
[104] Reidemeister, K., Einführung die kombinatorische Topologie, Braunschweig, Berlin (1932); reprint Chelsea, New York (1950).

[105] Smith, J., 'Equivariant Moore spaces', Algebraic and Geometric Topology: Proceedings Rutgers 1983, Ed. A. Ranicki, N. Levitt, F. Quinn, Springer Lecture Notes in Math 1126 (1985) 238-270.

[106] Spencer, C.B., 'An abstract setting for homotopy pushouts and pullbacks', Cah. Top. Géom. Diff., 18 (1977) 409-430.

[107] Spencer, C.B. and Wong, Y.L., 'Pullback and pushout squares in a special double category with connection' Cah. Top. Géom. Diff., 24 (1983) 161-192.

[108] Steiner, R. 'The algebra of directed complexes', Appl. Categorical Structures, 1 (1993) 247-284.

[109] Street, R., 'Categorical structures', in Handbook of algebra, edited M. Hazewinkel, Elsevier, Amsterdam (1996) 529-577.

[110] Street, R., 'Higher categories, strings, cubes and simplex equations', Appl. Categorical Structures, 3 (1995) 29-77.

[111] Taylor, J., 'Quotients of groupoids by the actions of a group', Math. Proc. Camb. Phil. Soc., 103 (1988) 239-249.

[112] Tonks, A.P., Theory and applications of crossed complexes, PhD thesis, University of Wales, Bangor (1993) http://www.bangor.ac.uk/ma/research/tonks.

[113] Weinstein, A., 'Groupoids: relating internal and external symmetry', Notices Amer. Math. Soc., 43 (7) (1996) 744-752.

[114] Wensley, C.D. and Alp, M., XMOD, a GAP share package for computation with crossed modules, (1997) GAP Manual, 1355-1420.

[115] Whitehead, G.W., Elements of homotopy theory, Springer, 1978.

[116] Whitehead, J.H.C., 'On adding relations to homotopy groups', Ann. Math. 42 (1941) 409-428.

[117] Whitehead, J.H.C., 'Note on a previous paper entitled "On adding relations to homotopy groups", Ann. Math. 47 (1946) 806-810.

[118] Whitehead, J.H.C., 'Combinatorial homotopy II', Bull. Amer. Math. Soc. 55 (1949) 453-496.

[119] Whitehead, J.H.C., 'A certain exact sequence', Ann. Math. 52 (1950) 51-110.

This article may be accessed via WWW at http://www.rmi.acnet.ge/hha/ or by anonymous FTP at ftp://ftp.rmi.acnet.ge/pub/hha/volumes/1999/n1.dvi (dvi, ps) 